Centre de Recherches Mathématiques CRM Proceedings and Lecture Notes

Volume 50,2010

\title{
From Minimal Geodesics to Supersymmetric Field Theories
}

\author{
Henning Hohnhold, Stephan Stolz, and Peter Teichner
}

\author{
In memory of Raoul Bott, friend and mentor.
}

\begin{abstract}
There are many models for the $K$-theory spectrum known today, each one having its own history and applications. The purpose of this note is to give an elementary description of eight such models (and certain completions of them) and to relate all of them by canonical maps, some of which are homeomorphisms (rather than just homotopy equivalences). Our survey begins with Raoul Bott's iterated spaces of minimal geodesics in orthogonal groups, which he used to prove his famous periodicity theorem, and includes Milnor's spaces of Clifford module structures as well as the Atyiah-Singer spaces of Fredholm operators. From these classical descriptions we move via spaces of unbounded operators and super-semigroups of operators to our most recent model, which is given by certain spaces of supersymmetric (1|1)-dimensional field theories. These spaces were introduced by the second two authors for the purpose of generalizing them to spaces of certain supersymmetric (2|1)dimensional Euclidean field theories that are conjectured to be related to the Hopkins - Miller spectrum TMF of topological modular forms.
\end{abstract}

\section{Introduction}

At the first Arbeitstagung 1957 in Bonn, Alexander Grothendieck presented his version of the Riemann-Roch theorem in terms of a group (now known as the Grothendieck group) constructed from (isomorphism classes of) algebraic vector bundles over algebraic manifolds. Some people say that he used the letter K to abbreviate 'Klassen,' the German word for (isomorphism) classes. Michael Atiyah and Friedrich Hirzebruch instantly realized that the same construction can be applied to all complex vector bundles over a topological space $X$, yielding a commutative ring $K(X)$, where addition and multiplication come from direct sum, respectively tensor product, of vector bundles. For example, every complex vector bundle over

2000 Mathematics Subject Classification. Primary 19L99; Secondary 18D10, 47A53, 57R56.

Key words and phrases. Differential geometry, algebraic geometry.

The second and third author were supported by the Max-Planck Society and grants from the NSF.

This is the final form of the paper.

(C)2010 American Mathematical Society 
the circle is trivial and hence $K\left(S^{1}\right)=\mathbb{Z}$. Moreover,

$$
K\left(S^{2}\right)=\mathbb{Z}[L] /(L-1)^{2},
$$

is generated by Hopf's line bundle $L$ over the 2 -sphere.

At the second Arbeitstagung in 1958, Raoul Bott explained his celebrated periodicity theorem, which can be expressed as the computations for all $n$ :

$$
K\left(S^{2 n-1}\right) \cong K\left(S^{1}\right) \cong \mathbb{Z} \text { and } K\left(S^{2 n}\right) \cong K\left(S^{2}\right) \cong \mathbb{Z}^{2}
$$

Bott also proved a real periodicity theorem involving the Grothendieck group of (isomorphisms classes of) real vector bundles over $X$. After dividing by the subgroup generated by trivial bundles (the quotient is denoted by a tilde over the $K$-groups) one obtains

$$
\widetilde{K O}\left(S^{n}\right) \cong \begin{cases}\mathbb{Z} & \text { for } n \equiv 0 \bmod 4 \\ \mathbb{Z} / 2 & \text { for } n \equiv 1,2 \bmod 8 \\ 0 & \text { else }\end{cases}
$$

It was again Atiyah and Hirzebruch who realized that Bott's periodicity theorem could be used to define generalized cohomology theories $K^{n}(X)$ and $K O^{n}(X)$ that are 2-periodic, respectively 8-periodic, and satisfy $K^{0}=K, K O^{0}=K O$. They satisfy the same Eilenberg-Steenrod axioms (functoriality, homotopy invariance and Mayer-Vietoris principle) as the ordinary cohomology groups $H^{n}(X)$ but if one takes $X$ to be a point one obtains nontrivial groups for some $n \neq 0$. In fact, the above computation yields by the suspension isomorphism

$$
K^{-n}(\mathrm{pt}) \cong \widetilde{K}^{-n}\left(S^{0}\right) \cong \widetilde{K}^{0}\left(S^{n}\right) \cong \begin{cases}\mathbb{Z} & \text { for } n \text { even } \\ 0 & \text { for } n \text { odd }\end{cases}
$$

and similarly for real $K$-theory. Several classical problems in topology were solved using this new cohomology theory. For example, the maximal number of independent vector fields on the $n$-sphere was determined explicitly. A modern way to express any generalized cohomology theory is to write down a spectrum, i.e., a sequence of pointed $\mathrm{CW}$-complexes $E_{n}$ with structure maps $\Sigma E_{n} \rightarrow E_{n+1}$.

For ordinary cohomology, these $E_{n}$ would be the Eilenberg-Mac Lane spaces $K(\mathbb{Z}, n)$. The purpose of this note is to give an elementary description of eight models (and their completions) of the spaces $E_{n}$ in the $K$-theory spectrum and relate all of them by canonical maps, most of which are homeomorphisms (rather then just homotopy equivalences). We will exclusively work with real $K$-theory $K O$ but all statements and proofs carry over to the complex case, and, with a little more care, also to Atiyah's Real $K$-theory.

Before we start a more precise discussion, we'll give a list of the models that will be described in this paper. Recall that a pointed CW-complex $E_{n}$ is said to represent the functor $K O^{n}$ if there are natural isomorphisms of pointed sets

$$
\left[X, E_{n}\right] \cong K O^{n}(X)
$$

for all CW-complexes $X$. By Brown's representation theorem, such $E_{n}$ exist and are unique up to homotopy equivalence. The suspension isomorphism $\overline{K O}^{n}\left(E_{n}\right) \cong$ $\widetilde{K O}^{n+1}\left(\Sigma E_{n}\right)$ in real $K$-theory then takes the identity map on $E_{n}$ to a map $\Sigma E_{n} \rightarrow$ $E_{n+1}$ whose adjoint must be a homotopy equivalence

$$
\epsilon_{n}: E_{n} \stackrel{\simeq}{\rightarrow} \Omega E_{n+1} \text {. }
$$


The sequence $\left\{E_{n}, \epsilon_{n}\right\}_{n \in \mathbb{Z}}$ of spaces and structure maps is a special type of spectrum, namely an $\Omega$-spectrum, that represents the cohomology theory $K O$. The homotopy groups of the $\Omega$-spectrum are then given by the following connected components:

$$
\pi_{n} K O:=K O^{n}(\mathrm{pt})=K O^{-n}(\mathrm{pt}) \cong \pi_{0} E_{-n}
$$

which explains partially why we describe these negatively indexed spaces below. Note that for $n \geq 0$, we also have $\pi_{0} E_{-n} \cong \pi_{0} \Omega^{n} E_{0} \cong \pi_{n} E_{0}$ but since we are considering periodic $K O$-theory, the spectrum has nontrivial negative homotopy groups.

To fix notation, let $C_{n}$ be the Clifford algebra associated to the positive definite inner product on $\mathbb{R}^{n}$. It has generators $e_{1}, \ldots, e_{n}$ satisfying the relations

$$
e_{i}^{2}=-\mathbb{1}, \quad e_{i} e_{j}+e_{j} e_{i}=0 \quad \text { for } i \neq j
$$

and it turns into a $C^{*}$-algebra via $e_{i}^{*}=-e_{i}$. For $n \geq 0$, we define $C_{-n}$ to be the Clifford $C^{*}$-algebra for the negative definite inner product, so the operators $e_{i}$ are self-adjoint and $e_{i}^{2}=\mathbb{1}$. We also fix a separable real Hilbert space $H_{n}$ with a $*$-representation of $C_{n-1}$ such that all (i.e., one or two) irreducible $C_{n-1^{-}}$ modules appear infinitely often. Note that $C_{n}$ is a $\mathbb{Z} / 2$-graded algebra such that the even part $C_{n}^{\text {ev }}$ is isomorphic to $C_{n-1}$. Thus we get a $\mathbb{Z} / 2$-graded $C_{n}$-module $\mathcal{H}_{n}:=H_{n} \otimes_{C_{n}^{\text {ev }}} C_{n}$. We denote the grading involution by $\alpha$. All gradings in this paper are $\mathbb{Z} / 2$-gradings.

Main Theorem. The following spaces are all homotopy equivalent and represent the $(-n)$ th space in the periodic real KO-theory $\Omega$-spectrum. The spaces in (2) to (5) are homeomorphic and so are their "completed" partners (written in parentheses).

(1) The Bott space $\mathcal{B}_{n}$. Here $\mathcal{B}_{1}$ is the union of all orthogonal groups $\mathrm{O}(k)$ where $k \in \mathbb{N}$. For $n>1$, the space $\mathcal{B}_{n}$ is the space of minimal geodesics in $\mathcal{B}_{n-1}$.

(2) The Milnor space $\mathcal{M}_{n}^{\text {fin }}$ (respectively $\mathcal{M}_{n}$ ) of " $C_{n-1}$-module structures" on $H_{n}$. More precisely, for $n>1$ these are unitary structures $J$ on $H_{n}$, such that $J-e_{n-1}$ has finite rank (is compact) and $J e_{i}=-e_{i} J$ for $1 \leq i \leq n-2$.

(3) The space $\operatorname{Inf}_{n}^{\text {fin }}$ (respectively $\operatorname{Inf}_{n}$ ) of "infinitesimal generators", i.e., odd, self-adjoint unbounded $C_{n}$-linear operators on $\mathcal{H}_{n}$ with finite rank (compact) resolvent.

(4) The configuration space $\operatorname{Conf}_{n}^{\text {fin }}$ (respectively Conf $_{n}$ ) of finite-dimensional ungraded mutually perpendicular $C_{n}$-submodules $V_{\lambda}$ of $\mathcal{H}_{n}$, labelled by finitely (discretely) many $\lambda \in \mathbb{R}$ satisfying $V_{-\lambda}=\alpha\left(V_{\lambda}\right)$.

(5) The space $\mathcal{S G O}_{n}^{\text {fin }}$ (respectively $\mathcal{S G O}_{n}$ ) of super-semigroups of self-adjoint $C_{n}$-linear finite rank (compact) operators on $\mathcal{H}_{n}$.

(6) The classifying space of the internal groupoid $1 \mid 1-\mathrm{PEFT}^{-n}$ of supersymmetric, positive Euclidean Field Theories of dimension (1|1) and degree $(-n)$.

(7) The classifying space $Q_{n}$ of a category that arises from a $C_{n}$-module category by a topological version of Quillen's $S^{-1} S$-construction.

(8) The Atiyah-Singer space $\mathcal{F}_{n}$ of certain skew-adjoint Fredholm operators on $H_{n}$, anticommuting with the $C_{n-1}$-action.

The above theorem only gives very rough descriptions of the spaces involved. Detailed definitions for each item $(k), k=1, \ldots, 8$, can be found in Section $k$ 
below. Section $k$ also contains the proof that the spaces in $(k)$ are homeomorphic (respectively homotopy equivalent) to spaces appearing previously.

To the best of our knowledge, the homeomorphisms between the spaces in (2), (4), (5) are new, even though it was well known that the spaces are homotopy equivalent for abstract reasons (since they represent the same $\Omega$-spectrum and have the homotopy type of a CW complex; in fact, our results imply that this is the case for all the spaces in our Main Theorem). Moreover, our maps relating the spaces in (4) and (7) respectively (3) and (8) seem to be new and slightly easier than the original ones. Another new aspect is the precise treatment of super-semigroups and the spaces $\mathcal{S G O}_{n}$.

Finally, the main new result is the equivalence of the older descriptions of $K$ theory with the one in (6). This paper does not contain the definitive treatment of this equivalence, we only outline the case for $n=0$, see $[12,29]$ for more details. Studying supersymmetric Euclidean field theories is a long term project of the last two authors, attempting to give a geometric description of the Hopkins-Miller theory of topological modular forms, the "universal" elliptic cohomology theory, by raising the dimension of the worldsheet from (1|1) to $(2 \mid 1)$.

Remark 0.1. The spaces in (3) to (7) are defined for all $n \in \mathbb{Z}$ and the theorem holds for all integers $n$. The Bott and Milnor spaces only make sense for $n \geq 1$ and the same seems to be true for the spaces in (8). This comes from the fact that the Atiyah-Singer spaces $\mathcal{F}_{n}$ are defined in terms of the ungraded Hilbert space $H_{n}$ and for $n \leq 0$ our translation to $\mathcal{H}_{n}$ doesn't work well. However, this can be circumvented by never mentioning the Clifford algebra $C_{n-1}$ in the definitions and working with the ungraded algebra $C_{n}^{\text {ev }}$ instead. Then the spaces $\mathcal{F}_{n}$ are defined for all $n \in \mathbb{Z}$ and our theorem holds. We chose the formulation above to better connect with the reference [2].

We should also mention some other descriptions of the spaces in the $\mathrm{KO}$ theory spectrum; we apologize in advance for any omissions. In [26], Graeme Segal introduces the technology of $\Gamma$-spaces to construct a connective spectrum from a symmetric monoidal category. The 0 th space $E_{0}$ in the resulting $\Omega$-spectrum is the group completion of the commutative $\mathrm{H}$-space resulting from the classifying space of the symmetric monoidal category. If one applies his machine to finite-dimensional vector spaces (with direct sum operation), one obtains connective ko-theory. This means that for $n \geq 0$ the spaces $E_{-n}=\Omega^{n} E_{0}$ are homotopy equivalent to the ones in our theorem but $E_{n}$ is $(n-1)$-connected and hence the negative homotopy groups of the spectrum vanish.

In his book on $K$-theory [14, Theorem III.4.27], Max Karoubi describes the $n$th space for $K O$ as the space of gradings of $\mathcal{H}_{n}$. These are orthogonal involutions that anticommute with the $e_{i}$ (and have no relation with the given grading $\alpha$ ).

In [10], Guentner and Higson use for the $(-n)$ th space for $K O$ all $\mathbb{Z} / 2$-graded $C^{*}$-algebra homomorphisms from $C_{0}(\mathbb{R})$ to the $C_{n}$-linear compact operators on $\mathcal{H}_{n}$. Here the $C^{*}$-algebra of continuous functions on $\mathbb{R}$ that vanish at $\infty$ is graded via the map $x \mapsto-x$ for $x \in \mathbb{R}$. We showed in [28, Lemma 3.15] that this space is actually homeomorphic to $\mathcal{S G O}_{n}$ in (5) above. This follows from the fact that $C_{0}(\mathbb{R})$ is the graded $C^{*}$-algebra generated by the super-semigroup $\mathbb{R}_{>0}^{1 \mid 1}$ used in the definition of $\mathcal{S G O}_{n}$.

In Pokman Cheung's thesis [5], another version of the $(-n)$ th space in the real $K$-theory spectrum was introduced. The author starts with $\operatorname{Conf}_{n}$ as a discrete set 
of objects, introduces a space of morphisms which gives a topological category and shows that its classifying space is homotopy equivalent to the Atiyah-Singer space $\mathcal{F}_{n}$. We shall compare his results with ours in Remark 7.3.

Survey of the spaces in our Main Theorem. We now give a rough outline of where the spaces come from and how they are related. The spaces $\mathcal{B}_{n}$ were defined by Raoul Bott in his classical paper [4] on "the stable homotopy of the classical groups" whose periodicity theorem is the heart and soul of $K$-theory. This model actually predates the invention of $K$-theory as a generalized cohomology theory, but was used by Bott to completely calculate the coefficients of this theory.

Atiyah, Bott, and Shapiro [1] showed the significance of Clifford algebras in $K$-theory and they suggested to look for a proof of the periodicity theorem using Clifford algebras. A proof along these lines was then found by Wood in [31] and also by Milnor in his beautiful book [22] on Morse theory. We will recall in Section 1 how one can easily compute (iterated) spaces of minimal geodesics in the orthogonal group in terms of Clifford module structures on $H_{n}$. In Section 2 we'll define the Milnor spaces $\mathcal{M}_{n}$ to be a certain completion of these spaces of Clifford module structures. These new spaces no longer depend on a basis of $H_{n}$ but they have the same homotopy type as $\mathcal{B}_{n}$ by a theorem of Palais [23].

The configuration spaces $\operatorname{Conf}_{n}^{\text {fin }}$ are the easiest to work with because one can geometrically picture its points very well as a finite collection of real numbers $\lambda$, labelled by (finite-dimensional and pairwise orthogonal) $C_{n}$-submodules $V_{\lambda} \subset \mathcal{H}_{n}$ such that $V_{-\lambda}=\alpha\left(V_{\lambda}\right)$. In [27], Segal had introduced such configuration spaces $F(X)$ in the case $n=0$ (and ungraded) for any compact space $X$ (with basepoint $x_{0}$ ) instead of the real line, one-point compactified to $S^{1}$ (with $\infty$ as basepoint). The topology on $F(X)$ is such that if two points of $X$ collide then the corresponding labels (also known as subspaces of $H=H_{0}$ ) add and moreover, a point can move to $x_{0}$ and is then discarded. Segal proved that if $X$ is connected then one has isomorphisms

$$
k o_{n}\left(X, x_{0}\right) \cong \pi_{n} F\left(X, x_{0}\right) \cong \pi_{n+1} F\left(\Sigma X, x_{0}\right)
$$

In particular, it follows that for $n>0$ the space $F\left(S^{n}\right)$ is the $n$th space of the connective $k o$-theory $\Omega$-spectrum. However, for $n=0$ this is clearly not the case since $F\left(S^{0}\right)$ is the Grassmannian of finite-dimensional subspaces of $H$ and hence has the homotopy type of $\coprod_{k} B O(k)$. As a consequence, one needs to group complete this space (with respect to direct sum) to obtain the correct homotopy type $B O \times$ $\mathbb{Z}$. Our graded version Conf fin of Segal's configuration space $F\left(S^{0}\right)$ already has this homotopy type and hence it should be thought of as a "group completion of configurations". We believe that more generally, if $X$ is connected then $F(X)=$ $F(X ; H)$ is homotopy equivalent to $\operatorname{Conf}\left(X \times S^{1},\left(x_{0}, \infty\right) ; \mathcal{H}\right)$. This is written in the notation of Section 4, where $\mathcal{H}=H \oplus H$ and the involution on $X \times(\mathbb{R} \cup \infty)$ is given by $(x, \lambda) \mapsto(x,-\lambda)$.

It came as a surprise to us that (equipped with the correct topology) the configuration space $\operatorname{Conf}_{n}^{\text {fin }}$ is actually the classifying space $Q_{n}$ of a certain (internal) Quillen category appearing as (7) in our Main Theorem. In the light of the above considerations regarding group completion, this was expected only for the homotopy type of the classifying space for $n=0$ and we shall outline in Section 7 how one can use Quillen's results to prove from this point of view that $\operatorname{Conf}_{0}^{\text {fin }}$ represents $K O^{0}$. 
Given a configuration $\left\{V_{\lambda}\right\}$ in $\operatorname{Conf}_{n}^{\text {fin }}$, one can interpret it as the eigenspaces and eigenvalues of an odd, self-adjoint, $C_{n}$-linear operator $\mathcal{D} \in \operatorname{Inf}_{n}^{\text {fin }}$ with domain $\bigoplus_{\lambda} V_{\lambda}$ that is given by

$$
\mathcal{D}(v):=\lambda \cdot v \quad \forall v \in V_{\lambda} .
$$

We note that the domain of $\mathcal{D}$ is finite-dimensional; in particular, $\mathcal{D}$ is not densely defined, a common requirement for self-adjoint operators in textbooks. We expand the usual definition of a self-adjoint (unbounded) operator by just requiring that the operator is self-adjoint on the closure of its domain. As a consequence, it is very natural to study completions $\operatorname{Conf}_{n}$ of the spaces Conf $f_{n}^{\text {fin }}$ where there is a discrete set of labels and hence the corresponding operator $\mathcal{D} \in \operatorname{Inf}_{n}$ may have dense domain (and it has compact resolvent). The resulting spaces $\operatorname{Inf}_{n}$ are equipped with the generalized norm topology and the fact that one can retract the completed spaces back to their finite rank subspaces goes back to (at least) Segal [27] but we re-prove this fact here.

The operator $\mathcal{D}$ can be used as the infinitesimal generator of the super-semigroup

$$
(t, \theta) \mapsto \mathrm{e}^{-t \mathcal{D}^{2}+\theta \mathcal{D}}
$$

of finite rank (respectively compact) operators on $\mathcal{H}_{n}$. These are the elements of $\mathcal{S G O}_{n}^{\text {fin }}$ (respectively $\mathcal{S G O}_{n}$ ). Here $(t, \theta) \in \mathbb{R}_{>0}^{1 \mid 1}$ parametrize a certain supersemigroup whose super Lie algebra is free on one odd generator. Such supersemigroups of operators should be considered the 'fermionic' or 'odd' analogue of usual semigroups of operators.

The homeomorphism from Conf ${ }_{n}^{\text {in }}$ to $\mathcal{M}_{n}$ is basically given by applying the inverse of the Cayley transform to the operator $\mathcal{D}$. If one applies this transformation to elements in Conf $_{n}$ one obtains interesting completions of the Milnor and Bott spaces.

The spaces $\mathcal{S} \mathcal{G O}_{n}$ were introduced by two of us in [28] as super-semigroups of self-adjoint operators and at the time we thought of them as Euclidean field theories, without having a precise definition for the latter. In the meantime, we have developed a precise notion of supersymmetric Euclidean field theories of dimension $d \mid \delta$, see [29]. These are certain fibred functors from a Euclidean bordism category to a category of topological vector spaces, both fibred over the site of complex super manifolds, see Section 6. Using invertible natural transformations between such functors leads to a groupoid of Euclidean field theories. Using the inner Hom in fibred categories, one naturally gets a groupoid internal in topological spaces and hence one has a classifying space. In Section 6 we will also assume that the Euclidean field theories are unitary and strongly positive and for $d|\delta=1| 1$ we denote the resulting classifying space by $1 \mid 1-\mathrm{PEFT}^{-n}$. Here the superscript refers to a degree $-n$ twist that will not be explained in this paper but see [11].

If one starts with a closed $n$-dimensional spin manifold $M$ then the $C_{n}$-linear Dirac operator $D_{M}$ (called Atiyah-Singer operator in [17, p. 140]) is an example of a (nonfinite) element in $\operatorname{Inf}_{n}$, where $\mathcal{H}_{n}$ are the $L^{2}$-sections of the $C_{n}$-linear spinor bundle on $M$. One can think of this operator as the infinitesimal generator of a supersymmetric (1|1)-dimensional quantum field theory, with Euclidean (rather than Minkowski) signature. This is the reason why we use the terminology Euclidean field theory. Actually, physicists would call it supersymmetric quantum mechanics on $M$, not a field theory, since space is 0-dimensional. 
The spaces $\mathcal{F}_{n}$ first appeared in the article [2] by Atiyah-Singer and are probably the most common model for $K$-theory. They make all the wonderful applications to analysis possible. From our point of view, the connection is easiest to make with the space $\operatorname{Inf}_{n}$ : Starting with a skew-adjoint Fredholm operator $T_{0}$ on $H_{n}$ that anticommutes with $e_{1}, \ldots, e_{n-1}$, we can turn it into an odd, self-adjoint, $C_{n}$-linear Fredholm operator

$$
T=T_{0} \otimes e_{n} \quad \text { or equivalently } \quad T=\left(\begin{array}{cc}
0 & T_{0}^{*} \\
T_{0} & 0
\end{array}\right)
$$

on $\mathcal{H}_{n} \cong H_{n} \oplus H_{n}$. It is easy to see that the map $T_{0} \mapsto T$ is a homeomorphism and it is important to note that the skew-symmetry of $T_{0}$ is equivalent to the relation $T e_{n}=e_{n} T$. This correspondence actually extends to the well known case $n=0$ where one starts with all Fredholm operators on $H_{0}$ and gets all odd, self-adjoint Fredholm operators on $H_{0} \oplus H_{0}$.

The essential spectrum of a Fredholm operator has a gap around zero and hence one can push the essential spectrum outside zero all the way to $\pm \infty$ by a homotopy. This turns a bounded operator into an unbounded one and is the basic step in the homotopy equivalence that takes a Fredholm operator $T$ to an infinitesimal generator $\mathcal{D}$. In the analytic literature, one can sometimes find concrete formulas in terms of functional calculus (which just describes the movement of the spectrum of $T$ ) like

$$
T=\frac{\mathcal{D}}{1+\mathcal{D}^{2}}
$$

Such precise formulas are not important from our point of view but the following subtlety arises in the operator $\mathcal{D}$ : its eigenspace at $\infty$, by definition the orthogonal complement of the domain of $\mathcal{D}$, is decomposed into the parts at $+\infty$, respectively $-\infty$. Such a datum is not present in general elements of $\operatorname{Inf}_{n}$ and it reflects the fact that we started with a bounded operator. Roughly speaking, this represents no problem up to homotopy if both these parts at $\pm \infty$ are infinite-dimensional. This uses Kuiper's theorem and is the only nonelementary aspect of this paper.

Taking into account the $C_{n}$-action, this is related to the following well-known subtlety in the Atiyah-Singer spaces of Fredholm operators. If $n \not \equiv 3 \bmod 4$, the spaces $\mathcal{F}_{n}$ are given by operators $T_{0}$ (or equivalently $T$ ) as above. However, Atiyah-Singer showed that for $n \equiv 3 \bmod 4$ the space of $C_{n-1}$-antilinear skewadjoint Fredholm operators on $H_{n}$ has two boring, contractible components $\widehat{\mathcal{F}}_{n}^{ \pm}$ consisting of operators $T_{0}$ such that

$$
e_{1} \cdot e_{2} \cdots e_{n-1} \cdot T_{0}
$$

is essentially positive (respectively negative). Recall that an operator is essentially positive if it is positive on a closed invariant subspace of finite codimension. So in the precise version for $\mathcal{F}_{n}$ in [2], the two components $\widehat{\mathcal{F}}_{n}^{ \pm}$are disregarded. It turns out that the above functional calculus leads to a map of all $C_{n-1}$-antilinear skewadjoint Fredholm operators to our spaces $\operatorname{Inf}_{n}$ but this map is a quasifibration (with contractible fibres) only on the component $\mathcal{F}_{n}$. Hence our spaces automatically remove the need for thinking about the above subtleties that arise from bounded operators and are interestingly only visible in the presence of special Clifford actions.

A symmetric ring spectrum for $\boldsymbol{K}$-theory. We end this introduction by explaining the easiest description (that we know) of a symmetric ring spectrum 
that represents $K$-theory. Let $\mathcal{H}_{-1}$ be a $C_{-1}$-module as in the paragraph preceding our Main Theorem, in particular it contains a submodule $V$ that is isomorphic to $C_{-1}$ as a $C_{-1}$-module. Then $\mathcal{H}_{-n}:=\mathcal{H}_{-1}^{\otimes n}$ has the properties required in our Main Theorem and hence there are corresponding spaces $E_{n}:=\operatorname{Inf}_{-n}^{\mathrm{fin}}\left(\mathcal{H}_{-n}\right)$ of infinitesimal generators as in (3) of that theorem. One can also use the completed version $\operatorname{Inf}_{-n}$ instead. We point out that $E_{n}$ contains a canonical base point, namely the operator whose domain is zero (and thus all eigenvalues are at $\infty$ ).

Theorem 0.2. For $n \geq 0$, the spaces $E_{n}$ form a symmetric ring spectrum representing real $K$-theory. The relevant structures are given as follows.

- The symmetric group $\Sigma_{n}$ acts by permuting the $n$ tensor factors of $\mathcal{H}_{-n}$.

- The multiplication maps $E_{n} \wedge E_{m} \rightarrow E_{n+m}$ are given by the formula

$$
\left(D_{n}, D_{m}\right) \mapsto D_{n} \otimes \mathbb{1}+\mathbb{1} \otimes D_{m}
$$

- The $\Sigma_{n}$-equivariant structure maps $\mathbb{R}^{n} \rightarrow E_{n}$ are given by sending $v \in \mathbb{R}^{n}$ to its Clifford action on the $C_{-n}$-module $V^{\otimes n}$ (and $\infty$ on the orthogonal complement in $\left.\mathcal{H}_{-n}\right)$. As $v \rightarrow \infty$, Clifford multiplication also goes to $\infty$ and hence the structure maps can be extended to $S^{n}$, sending the point at $\infty$ to the base point in $E_{n}$. These operators are odd and self-adjoint which explains the negative sign of $-n$.

This result is a reformulation of a theorem of Michael Joachim [13], so we shall not give a proof. By using complex Hilbert spaces and Clifford algebras, all our results translate to complex $K$-theory. In fact, keeping track of the involution of complex conjugation, one also gets Atiyah's Real $K$-theory which contains both real $K$-theory (via taking fixed points) and complex $K$-theory (by forgetting the conjugation map).

\section{Bott spaces of minimal geodesics}

The origin of topological $K$-theory is Raoul Bott's classical paper [4] on "The stable homotopy of the classical groups". For a Riemannian manifold $M$, let $v=$ $(P, Q, h)$ denote a 'base point in $M$ ' which is actually a pair of points $P, Q \in M$, together with a fixed homotopy class $h$ of paths connecting $P$ and $Q$. If $P=Q$ then $h$ is just an element in $\pi_{1}(M, P)$.

Bott considers the space $M^{v}$ of minimal geodesics from $P$ to $Q$ in the homotopy class $h$, a subspace of $\Omega_{v}$, the space of all such paths. Let $|v|$ be the first positive integer which occurs as the index of some geodesic with base point $v$. Then Bott proved the following theorem in [4]:

Theorem 1.1 (Bott). If $M$ is a symmetric space, so is $M^{v}$. Moreover, the based loop space $\Omega_{v}$ can be built, up to homotopy, by starting with $M^{v}$ and attaching cells of dimension $\geq|v|$ :

$$
\Omega_{v} \simeq M^{v} \cup \mathrm{e}^{|v|} \cup(\text { higher-dimensional cells }) \text { written as } M^{v} \stackrel{|v|}{\longrightarrow} M .
$$

For example, if $M=S^{n}, n>1$, with the round metric and $P, Q$ are not antipodal, then there is a unique minimal geodesic from $P$ to $Q$. The second shortest geodesic from $P$ to $Q$ reaches the point $-Q$ that has an $(n-1)$-dimensional variation of geodesics to $Q$ and hence $|v|=n-1$. In the notation above, one gets

$$
\text { pt }=M^{v} \stackrel{(n-1)}{\longrightarrow} M=S^{n}
$$

This is a free offprint provided to the author by the publisher. Copyright restrictions may apply. 
which implies that $\Omega S^{n}$ is $(n-2)$-connected, or equivalently, that $S^{n}$ is $(n-1)$ connected, not such a great result. However, if one considers all geodesics, one can say much more. In fact, the indices of geodesics from $P$ to $Q$ are $k(n-1)$ for $k=0,1,2, \ldots$. This is a case of Morse's original application of his theory to infinite-dimensional manifolds: the energy functional

$$
E: \Omega_{v} M \rightarrow \mathbb{R}, \quad E(\gamma):=\int_{0}^{1}\left|\gamma^{\prime}(t)\right|^{2} \mathrm{~d} t
$$

is a Morse function with critical points the geodesics and indices given by the number of conjugate points (counted with multiplicity) along the given geodesic. Morse shows that infinite-dimensionality is not an issue, because the space of paths with bounded energy has the homotopy type of a finite-dimensional space, namely the piecewise geodesics (where the number of corners is related to the energy bound and the injectivity radius of $M)$. As a consequence of our example above,

$$
\Omega_{v} S^{n} \simeq S^{n-1} \cup \mathrm{e}^{2(n-1)} \cup \mathrm{e}^{3(n-1)} \cup \cdots
$$

If $P$ and $Q$ are antipodal points on $S^{n}$, then the energy is not a Morse function. For example, the minimal geodesics form an $(n-1)$-sphere, parametrized by the equator in $S^{n}$. However, Bott developed a theory for such cases, now known as Morse-Bott theory, where the critical points form a submanifold whose tangent space equals the null space of the Hessian of the given function, the Morse-Bott condition. Applied to the case at hand, we can derive the same cell decomposition of $\Omega_{v} S^{n}$ as above but this time the bottom cell consists of the minimal geodesics:

$$
S^{n-1}=M^{v} \stackrel{2(n-1)}{\longrightarrow} M=S^{n}
$$

So Freudenthal's suspension theorem is a direct consequence of this result, using only the index of the first nonminimal geodesics (not the first two, as in the generic case studied by Morse). More generally, for any symmetric space, this is Bott's proof of Theorem 1.1 above.

His approach to study the homotopy types of the classical groups was to apply this method to compact Lie groups which are symmetric spaces in their bi-invariant metric. For example, consider $M=\mathrm{O}(2 m)$ and $P=\mathbb{1}, Q=-\mathbb{1}$. Then every geodesic $\gamma$ from $P$ to $Q$ is of the form

$$
\gamma(t)=\exp (\pi t \cdot A), \quad t \in[0,1]
$$

where $A$ is skew-adjoint. Thus we can 'diagonalize' $A$ by an orthogonal matrix $T$, i.e., $T A T^{-1}$ is a block sum $B$ of matrices

$$
\left(\begin{array}{cc}
0 & a_{i} \\
-a_{i} & 0
\end{array}\right)
$$

with $a_{1}, \ldots, a_{m} \geq 0$ after normalization. Since $\gamma(1)=-\mathbb{1}$, we see that the $a_{i}$ are odd integers. It is not hard to see that the energy of $\gamma$ is given by the formula

$$
E(\gamma)=2\left(a_{1}^{2}+\cdots+a_{m}^{2}\right)
$$

so that minimal energy (or equivalently, minimal length) means that all $a_{i}=1$. We note that for $m>1$, the energy determines the homotopy class $h$ of such a path so that we don't need to mention it for minimal energy (or length) paths (this stays true in all considerations below as well). We conclude that

$$
A^{2}=T^{-1} B^{2} T=T^{-1}(-\mathbb{1}) T=-\mathbb{1} \quad \text { and } \quad A^{*}=-A=A^{-1}
$$


so that $A$ is a complex structure on $\mathbb{R}^{2 m}$. Just like for the standard complex structure we have

$$
A=\exp ((\pi / 2) \cdot A)=\gamma(1 / 2)
$$

and we obtain the following result.

Proposition 1.2 (Bott). The space $\mathcal{B}_{2}(2 m)$ of minimal geodesics in $\mathrm{O}(2 \mathrm{~m})$ with basepoint $v$ as above is isometric to the space $\mathcal{M}_{2}(2 m)$ of unitary structures on $\mathbb{R}^{2 m}$ (consisting of $J \in \mathrm{O}(2 m)$ with $J^{2}=-\mathbb{1}$ ). Moreover, this is a totally geodesic submanifold of $\mathrm{O}(2 \mathrm{~m})$ and the homeomorphism is given by sending a geodesic $\gamma$ to its midpoint $\gamma\left(\frac{1}{2}\right)$.

We are introducing a notation that is consistent with

$$
\mathcal{B}_{1}(m)=\mathrm{O}(m)=\mathcal{M}_{1}(m)
$$

and will lead to the Bott and Milnor spaces in the limit when $m \mapsto \infty$. Now recall that $\mathcal{B}_{2}(2 m)$ is again a symmetric space by Bott's theorem so that we can iterate the construction: Pick a complex structure $J_{1}$ and study the space $\mathcal{B}_{3}(4 m)$ of minimal geodesics in $\mathcal{B}_{2}(4 \mathrm{~m})$ from $J_{1}$ to $-J_{1}$ (they automatically lie in a fixed homotopy class).

By a very similar discussion as above, it turns out that the midpoint map gives an isometry

$$
\mathcal{B}_{3}(4 m) \cong \mathcal{M}_{3}(4 m):=\left\{J \in \mathrm{O}(4 m) \mid J^{2}=-\mathbb{1}, J J_{1}=-J_{1} J\right\} .
$$

Note that the right-hand side is the space of (orthogonal) quaternion structures on $\mathbb{R}^{4 m}$ that are compatible with the given unitary (or orthogonal complex) structure $J_{1}$. The set of such structures form a totally geodesic submanifold of $\mathrm{O}(4 \mathrm{~m})$. More generally, we make the following

Definition 1.3. Assume that $\mathbb{R}^{m}$ is a $C_{n-2}$-module for some $n>1$. More precisely, there is a $*$-homomorphism $C_{n-2} \rightarrow \operatorname{End}\left(\mathbb{R}^{m}\right)$ sending $e_{i}$ to $J_{i}$ for $i=$ $1, \ldots, n$. Note that the compatibility with $*$ implies that $J_{i} \in \mathrm{O}(m)$. Then we define Milnor spaces

$$
\mathcal{M}_{n}(m):=\left\{J \in \mathrm{O}(m) \mid J^{2}=-\mathbb{1}, J J_{i}=-J_{i} J \forall i=1, \ldots, n-2\right\}
$$

to be "the space of all $C_{n-1}$-structures on $\mathbb{R}^{m}$ ", compatible with the given $C_{n-2^{-}}$ structure. Note that these spaces can be empty.

Proposition 1.4 (Bott). All $\mathcal{M}_{n}(m)$ are totally geodesic submanifolds of $\mathrm{O}(m)$. The space of minimal geodesics in $\mathcal{M}_{n}(m)$ from $J_{n-1}$ to $-J_{n-1}$ is isometric to $\mathcal{M}_{n+1}(m)$ via the midpoint map.

Proof. Let's assume the first sentence and show the second assertion. Any geodesic $\gamma$ from $J_{n-1}$ to $-J_{n-1}$ is of the form

$$
\gamma(t)=J_{n-1} \cdot \exp (\pi t \cdot A), \quad t \in[0,1]
$$

for some skew-adjoint matrix $A$. One checks that $\gamma\left(\frac{1}{2}\right)=J_{n-1} \cdot A$ has square $-\mathbb{1}$ and anticommutes with $J_{1}, \ldots J_{n-2}$ if and only if $\gamma$ lies in the submanifold $\mathcal{M}_{n}(m)$.

Definition 1.5. Inductively, let the Bott spaces $\mathcal{B}_{n+1}(m)$ be the space of minimal geodesics in $\mathcal{B}_{n}(m)$ from $J_{n-1}$ to $-J_{n-1}$ (for those $m$ where such a path exists). Then the previous discussion shows that the midpoint map gives an isometry

$$
\mathcal{B}_{n+1}(m) \cong \mathcal{M}_{n+1}(m)
$$


1.1. Bott periodicity. This leads to Bott's original proof of the celebrated periodicity theorems as follows.

Theorem 1.6 (Bott). Let $d_{n}$ be the minimal dimension of a $C_{n}$-module. Recall Bott's notation for the cell decomposition of the space of loops. Then

$$
\mathcal{B}_{n+1}(m) \stackrel{\frac{m}{d_{n}}-1}{\longrightarrow} \mathcal{B}_{n}(m)
$$

This implies in particular that for $m \rightarrow \infty$, the smallest dimension of a cell needed to get the loop space from the space of minimal geodesics also goes to infinity. Thus in the limit, one gets a homotopy equivalence

$$
\Omega_{v} \mathcal{B}_{n} \simeq \mathcal{B}_{n+1} .
$$

To make this precise, we define the spaces $\mathcal{M}_{n}(\infty)$, which were studied by Milnor in [22], as the union of all $\mathcal{M}_{n}(m)$ inside $\mathrm{O}(\infty)$ (which is the union of all $\mathrm{O}(m)$ ). In fact, we only take the union over those $m$ that are divisible by $d_{n}$. With a similar definition of $\mathcal{B}_{n}=\mathcal{B}_{n}(\infty)$, the midpoint maps give homeomorphisms

$$
\mathcal{B}_{n}(m) \approx \mathcal{M}_{n}(m) \quad \forall m=1,2, \ldots \text { (including } m=\infty \text { ). }
$$

between these Bott and Milnor spaces. Now by Morita equivalence $\mathcal{M}_{n}(\infty) \approx$ $\mathcal{M}_{n+8}(\infty)$ because $C_{n+8}$ is a real matrix ring over $C_{n}$. As a consequence,

Corollary 1.7 (Bott). There are homeomorphisms and homotopy equivalences

$$
\mathcal{B}_{n} \approx \mathcal{B}_{n+8} \simeq \Omega^{8} \mathcal{B}_{n}
$$

and the homotopy groups of $\mathrm{O}(\infty)$ are 8-periodic.

These groups are known as the 'stable' homotopy groups of the orthogonal group because

$$
\pi_{i} \mathrm{O}(m) \cong \pi_{i} \mathrm{O}(\infty) \quad \forall i<m-1
$$

\section{Milnor spaces of Clifford module structures}

For each $n \geq 1$, let $H_{n}$ be a separable Hilbert space that is a $C_{n-1}$-module such that each irreducible representation of $C_{n-1}$ appears with infinite multiplicity.

Definition 2.1. For $n=1$ we define

$$
\mathcal{M}_{1}^{\mathrm{fin}}=\left\{A \in \mathrm{O}\left(H_{1}\right) \mid A \equiv \mathbf{1} \text { modulo finite rank operators }\right\} \text {. }
$$

For $n \geq 2$ the (finite rank) Milnor space $\mathcal{M}_{n}^{\text {fin }}$ is the space of orthogonal operators $J$ on $H_{n}$ satisfying

- $J^{2}=-\mathbb{1}$, or, equivalently, $J$ is skew-adjoint,

- $J$ anticommutes with $e_{1}, \ldots, e_{n-2}$,

- $J-e_{n-1}$ has finite rank.

If we replace finite rank operators by compact operators in the above definition, we get the Milnor spaces $\mathcal{M}_{n}$ for $n \geq 1$.

The main result from the previous section is that the Bott spaces $\mathcal{B}_{n}$ are homeomorphic to the filtered union (with the direct limit topology)

$$
\mathcal{M}_{n}(\infty)=\bigcup_{k=1}^{\infty} \mathcal{M}_{n}\left(k \cdot d_{n}\right)
$$


i.e., they can be calculated in terms of spaces of Clifford algebra structures on $\mathbb{R}^{\infty}$, see Definition 1.3. Let $M$ be the unique irreducible $C_{n-1}$-module if $n$ is not divisible by 4 , respectively the sum of the two irreducible $C_{n-1}$-modules if $n$ is divisible by 4. We may choose embeddings

$$
H_{n}(m):=\bigoplus_{k=1}^{m} M \subset H_{n} \quad \forall m=1,2, \ldots \text { (including } m=\infty \text { ). }
$$

This is just the choice of an orthonormal basis in the case $n=1$. We get an embedding of $H_{n}(\infty)\left(=\mathbb{R}^{\infty}\right.$ for $\left.n=1\right)$ into $H_{n}$ and an induced inclusion of $\mathcal{M}_{n}(\infty)$ into $\mathcal{M}_{n}$.

Theorem 2.2. For all $n \geq 1$, this inclusion is a homotopy equivalence

$$
\mathcal{M}_{n}(\infty) \stackrel{\simeq}{\leftrightarrows} \mathcal{M}_{n} \text {. }
$$

It will follow from Proposition 4.6 that the inclusions $\mathcal{M}_{n}^{\text {fin }} \hookrightarrow \mathcal{M}_{n}$ are homotopy equivalences (and hence so are the inclusions $\mathcal{M}_{n}(\infty) \hookrightarrow \mathcal{M}_{n}^{\text {fin }}$ ). In fact, in the definition of the Milnor spaces, one can use any space of operators in between finite rank and compact operators (with the norm topology) to make this result true.

Proof. We shall use Palais' Theorem (A) from [23] which states the following. Let $E$ be a Banach space and $\pi(m)$ continuous projection operators onto finitedimensional subspaces $E(m) \subset E(m+1)$ which tend strongly to the identity as $m \rightarrow \infty$. Then for any open subspace $\mathcal{O}$ of $E$, the inclusion map

$$
\mathcal{O}(\infty) \hookrightarrow \mathcal{O}
$$

is a homotopy equivalence. Here $\mathcal{O}(\infty)$ is the direct limit of all $\mathcal{O}(m):=\mathcal{O} \cap E(m)$. In our setting, we have an extra parameter $n \geq 2$, where we leave the easiest case $n=1$ to the reader. We define

$$
E_{n}:=\left\{A \in \mathcal{K}\left(H_{n}\right) \mid A^{*}=-A, A e_{i}=-e_{i} A \text { for } i=1, \ldots, n-2\right\}
$$

where $\mathcal{K}\left(H_{n}\right)$ is the Banach space of compact operators on $H_{n}$ with the norm topology. Consider the $C_{n-1}$-linear orthogonal projections $H_{n} \rightarrow H_{n}(m)$ which by pre- and post-composition induce projections

$$
\pi(m): E_{n} \rightarrow E_{n}(m):=E_{n} \cap \mathcal{K}\left(H_{n}(m)\right) .
$$

We may assume that $H_{n}$ is the closure of $H_{n}(\infty)$ and hence that the $\pi(m)$ tend strongly to the identity. For the open subset in Palais' theorem we choose

$$
\mathcal{O}_{n}:=\left\{A \in E_{n} \mid A+e_{n-1} \text { is invertible }\right\}
$$

and $\mathcal{O}_{n}(m):=\mathcal{O}_{n} \cap E_{n}(m)$. Then the map $A \mapsto A+e_{n-1}$ gives homeomorphisms of $\mathcal{O}_{n}$, respectively $\mathcal{O}_{n}(m)$, with

$$
\mathcal{S}_{n}:=\left\{B \in \mathrm{GL}\left(H_{n}\right) \mid B-e_{n-1} \in E_{n}\right\}
$$

respectively $\mathcal{G}_{n}(m):=\mathcal{G}_{n} \cap \mathrm{GL}\left(H_{n}(m)\right)$. Palais' theorem says that the inclusion induces a homotopy equivalence

$$
\mathcal{G}_{n}(\infty) \stackrel{\simeq}{\hookrightarrow} \mathcal{G}_{n} .
$$

To prove our Theorem 2.2, we need to replace the general linear groups by the orthogonal groups in all of the above. This can be done by the polar decomposition of the invertible skew-adjoint operators $B$ above. We may write

$$
B=U \cdot P=P \cdot U
$$


where $P:=\sqrt{B^{*} B}=\sqrt{B B^{*}}$ is positive and $U$ is an orthogonal operator. Note that $U$ is actually also skew-adjoint:

$$
U^{*}=\left(B P^{-1}\right)^{*}=P^{-1} B^{*}=P^{-1}(-B)=-U
$$

Moreover, $B$ anticommutes with $e_{i}$ for $i=1, \ldots, n-2$ and therefore $P$ commutes with these $e_{i}$ and hence $U$ again anticommutes with them. Finally, it is easy to check that $U-e_{n-1}$ is a compact operator and hence $U$ has the same properties as $B$ and therefore lies in $\mathcal{M}_{n} \subset \mathcal{G}_{n}$. This implies that there is a commutative diagram

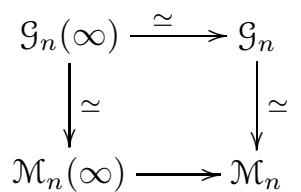

where the vertical maps are given by polar decomposition $B \mapsto U$. Since these are well known to be the homotopy inverses to the inclusion maps, the proof of Theorem 2.2 is completed.

\section{Infinitesimal generators}

In this section we will review some basic facts about self-adjoint (unbounded) operators, reminding the reader of a nice topology on this space. Let $H$ be a separable complex Hilbert space. Denote by Inf the set of all self-adjoint operators on $H$ with compact resolvent. Note that we do not require an element $\mathcal{D} \in \operatorname{Inf}$ to be densely defined. By 'self-adjoint' we mean that $\mathcal{D}$ defines a self-adjoint operator on the closure of its domain. The compact resolvent condition means that the spectrum of $\mathcal{D}$ consists of eigenvalues of finite multiplicity that do not have an accumulation point in $\mathbb{R}$. Hence, if the domain of $\mathcal{D}$ is infinite-dimensional, the operator $\mathcal{D}$ on $\overline{\operatorname{dom}(\mathcal{D})}$ is necessarily unbounded. Because of this, we will think of $\operatorname{dom}(\mathcal{D})^{\perp}$ as the eigenspace of $\mathcal{D}$ associated with the 'eigenvalue' $\infty$.

Functional calculus gives a bijection, see, e.g., [10],

$$
\operatorname{Inf} \leftrightarrow \operatorname{Hom}\left(C_{0}(\mathbb{R}), \mathcal{K}\right)
$$

where the right-hand side is the space of all $C^{*}$-homomorphisms from (complexvalued) continuous functions on $\mathbb{R}$ that vanish at $\infty$ to the compact operators $\mathcal{K}$ on $H$. Note that both of these $C^{*}$-algebras do not have a unit. Below, we will also deal with $C^{*}$-algebras that do have a unit and in this case Hom will denote those $C^{*}$-homomorphisms that preserve the unit.

We define the space $\widetilde{\text { Inf }}$ to be just as above, except that we do not require the spectrum to be discrete (and the eigenspaces can be infinite-dimensional). The "Cayley transform" is defined for such operators by functional calculus using the Möbius transformation

$$
c(x):=\frac{x+i}{x-i}
$$

which takes $\mathbb{R} \cup\{\infty\}$ to the unit circle $S^{1}$. It defines the mapping from the very left to the very right in the following theorem.

Theorem 3.1. There are bijections

$$
\widetilde{\operatorname{Inf}} \stackrel{a}{\leftrightarrow} \operatorname{Hom}\left(C_{0}(\mathbb{R}), \mathcal{B}\right) \stackrel{c}{\cong} \operatorname{Hom}\left(C\left(S^{1}\right), \mathcal{B}\right) \stackrel{b}{\leftrightarrow} \mathcal{U}
$$


where $\mathcal{B}$ and $\mathcal{U}$ are the bounded, respectively unitary, operators on $H$. Moreover, the bijection $b$ on the right, given by functional calculus, is a homeomorphism from the pointwise norm topology on $\operatorname{Hom}\left(C\left(S^{1}\right), \mathcal{B}\right)$ to the operator norm topology on U.

Definition 3.2. We give $\widetilde{\text { Inf }}$ the topology coming from the above bijections. This is sometimes referred to as the generalized norm topology because of Lemma 3.4 below.

Remark 3.3. Just like Inf has an interpretation in terms of configuration spaces, by using the pattern of eigenvalues and their eigenspaces, the space $\widetilde{\text { Inf }}$ can be interpreted as the space of all projection-valued measures on $\mathbb{R}$, see $[24$, Theorem VIII.6]. The fact that the operators may not be densely defined is reflected in the fact that the projection corresponding to all of $\mathbb{R}$ is not necessarily the identity but projects onto the domain. Thus the result becomes cleaner than in [24] where the map $b$ is not onto.

Theorem 3.1 is well known; we just need to collect various bits and pieces of the argument, for example from Rudin [25] or Reed-Simon [24]. These authors only define the adjoint of a densely defined operator $D$ because otherwise the adjoint is not determined by the formula

$$
\langle D v, w\rangle=\left\langle v, D^{*} w\right\rangle
$$

In particular, self-adjoint operators are assumed to have dense domain. As a consequence, [25, Theorem 13.19] proves that the Cayley transform gives an inclusion of all densely defined self-adjoint operators onto the space of unitary operators without eigenvalue 1 . If one allows nondense domains, i.e., eigenvalue $\infty$ (defining the adjoint also to be $\infty$ on that subspace), then the Cayley transform takes the eigenspace of $\infty$ to the eigenspace of 1 and therefore becomes onto all unitary operators, i.e., gives the desired bijection $\widetilde{\operatorname{Inf}} \leftrightarrow \mathcal{U}$.

Proof of Theorem 3.1. The map $a$ is given by functional calculus, which is well defined on self-adjoint operators that are densely defined. Since the functions $f$ vanish at $\infty$ one can extend this for all $D \in \widetilde{\operatorname{Inf}}$ by defining $f(D)$ to be zero on the orthogonal complement of the domain of $D$. For the second map, note that $C\left(S^{1}\right)$ is obtained from $C_{0}(\mathbb{R})$ by adding a unit $\mathbb{1}$ (and using the above Möbius transformation $c$ ). We get an isomorphism between the two spaces of $C^{*}$-homomorphisms since we require that $\mathbb{1}$ maps to $\mathbb{1}$ (if the algebras have units). Finally, the map $b$ is given by evaluating a homomorphism at the identity map $z: S^{1} \rightarrow S^{1}$. It is clear that the composition from left to right is therefore the Cayley transform and hence a bijection. Recall that by Fourier decomposition, there is an isomorphism of complex $C^{*}$-algebras

$$
C\left(S^{1}\right) \cong C^{*}(\mathbb{Z})
$$

where $\mathbb{Z}$ is the infinite cyclic group, freely generated by an element $z$ (which corresponds to the above identity $z$ on $S^{1}$ ). It follows that $C^{*}(\mathbb{Z})$ is free as a $C^{*}$-algebra on one unitary element $z$ and hence $C^{*}$-homomorphisms out of it are just unitary elements in the target. Moreover, the bijection is given by evaluating functions on this unitary $z$ which is our map $b$ above.

To show that $b$ is a homeomorphism, we need to show that a sequence $\varphi_{n}$ of $C^{*}$-homomorphisms converges if and only if $\varphi_{n}(z)$ converges (in norm). By 
definition, the $\varphi_{n}$ converge if $\varphi_{n}(f)$ converges (in norm) for all $f$, so one direction is obvious. For the other, assume that $u_{n}:=\varphi_{n}(z)$ converges to $u \in \mathcal{U}$ and note that $\varphi_{n}(f)=f\left(\varphi_{n}(z)\right)=f\left(u_{n}\right)$. We want to show that $f\left(u_{n}\right)$ converges to $f(u)$ and we claim that this is easy to check in the case when $f$ is a Laurent polynomial. For the general case, pick $\epsilon>0$ and choose a Laurent polynomial $p=p(z)$ such that $\|f-p\|_{\text {sup }}<\epsilon / 3$ and an $N \gg 0$ such that $\left\|p\left(u_{n}\right)-p(u)\right\|<\epsilon / 3$ for $n>N$. Then for $n>N$ we have

$$
\left\|f\left(u_{n}\right)-f(u)\right\|=\left\|\left(f\left(u_{n}\right)-p\left(u_{n}\right)\right)+\left(p\left(u_{n}\right)-p(u)\right)+(p(u)-f(u))\right\|<\epsilon
$$

and hence $f\left(u_{n}\right)$ converges to $f(u)$. This argument is very similar to the one in [24, Theorem VIII.20(a)].

Lemma 3.4. The Cayley transform on bounded operators

$$
\mathcal{B}^{\mathrm{sa}} \subset \widetilde{\mathrm{Inf}} \leftrightarrow \mathcal{U}
$$

is an open embedding, i.e., the generalized norm topology on $\widetilde{\operatorname{Inf}}$ extends the operator norm topology on $\mathcal{B}^{\text {sa }}$, the bounded self-adjoint operators on $H$.

Again this result is well known, see for example [24, Theorem VIII.18]. Reed and Simon use the resolvent instead of the Cayley transform but this is just a different choice of Möbius transformation, using $x \mapsto(x+i)^{-1}$ instead of $c$. This has the effect that the image of $\mathbb{R}$ is not the unit circle but a circle of radius $\frac{1}{2}$ inside the unit circle. Therefore, one does not get unitary operators but there is certainly no difference for the induced topology. Unfortunately, in the above Theorem VIII.18, Reed and Simon assume an additional property on the sequence considered, namely that it is uniformly bounded. It turns out, however, that this assumption is unnecessary, which is an easy consequence of Theorem VIII.23(b) in [24].

Remark 3.5. It is interesting to recall from [25, Theorem 13.19] that the Cayley transform can also be applied to symmetric operators, i.e., those that are formally self-adjoint and with $\operatorname{Dom}(D) \subseteq \operatorname{Dom}\left(D^{*}\right)$. The result is an isometry $U$ with

$$
\operatorname{Dom}(U)=\operatorname{Range}(D+i \cdot \mathbb{1}) \text { and } \operatorname{Range}(U)=\operatorname{Range}(D-i \cdot \mathbb{1})
$$

$D$ is closed if and only if $U$ is closed and $D$ is self-adjoint if and only if $U$ is unitary. Using the Cayley transform and its inverse one sees that the self-adjoint extensions of $D$ are in 1-1 correspondence with unitary isomorphisms between the orthogonal complements of $\operatorname{Dom}(U)$ and Range $(U)$. In particular, self-adjoint extensions exist if and only if these complements have the same dimensions, usually referred to as the deficiency indices.

An example to keep in mind is the infamous right shift, which is an isometry with deficiency indices 0 and 1 . Thus its inverse Cayley transform has no self-adjoint extension.

Let $\operatorname{Inf}^{\text {fin }}$ be the space of all self-adjoint operators on $H$, with finite spectrum and multiplicity (not necessarily densely defined).

Proposition 3.6. The Cayley transform induces the following bijections

$$
\begin{aligned}
& \operatorname{Inf} \stackrel{a}{\leftrightarrow} \operatorname{Hom}\left(C_{0}(\mathbb{R}), \mathcal{K}\right) \stackrel{c}{\cong} \operatorname{Hom}\left(C\left(S^{1}\right), \mathcal{K}+\mathbb{C} \cdot \mathbb{1}\right) \stackrel{b}{\leftrightarrow} \mathcal{U} \cap(\mathcal{K}+\mathbb{C} \cdot \mathbb{1}) \\
& \operatorname{Inf}^{\text {fin }} \stackrel{a}{\leftrightarrow} \operatorname{Hom}\left(C_{0}(\mathbb{R}), \mathcal{F R}\right) \stackrel{c}{\cong} \operatorname{Hom}\left(C\left(S^{1}\right), \mathcal{F R}+\mathbb{C} \cdot \mathbb{1}\right) \stackrel{b}{\leftrightarrow} \mathcal{U} \cap(\mathcal{F R}+\mathbb{C} \cdot \mathbb{1})
\end{aligned}
$$


where $\mathcal{K}$ and $\mathcal{F} \mathcal{R}$ are the compact, respectively finite rank, operators on $H$. Moreover, the bijections $b$ on the right, given by functional calculus, are homeomorphisms from the pointwise norm topology on the spaces of $C^{*}$-homomorphisms to the operator norm topology on $\mathrm{U}$.

Proof. The Cayley transforms give the bijections from the very left to the very right because one can read off the conditions of being compact, respectively finite rank, from the spectrum and multiplicities of the operators. These conditions are mapped into each other by definition of the spaces. The fact that the maps $b$ are homeomorphisms is proved exactly as in Theorem 3.1.

We now have complete control over the topology on our various spaces. The

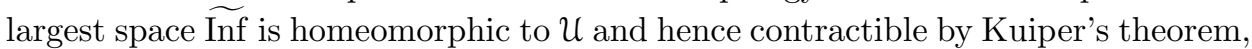
whereas the subspaces Inf ${ }^{\text {fin }}$ and Inf are homotopy equivalent (see Proposition 4.6) and have a very interesting topology.

We shall now add some bells and whistles, like grading, real structure and Clifford action to make these spaces even more interesting. In a first step, assume that our complex Hilbert space $H$ has a real structure, i.e., that

$$
H=H_{\mathbb{R}} \otimes_{\mathbb{R}} \mathbb{C}
$$

for some real Hilbert space $H_{\mathbb{R}}$. If we think of the real structure (also known as complex conjugation) on $H$ as a grading involution $\alpha$ (which has the property that the even and odd parts are isomorphic) then the above Proposition 3.6 leads to the following result.

Proposition 3.7. The Cayley transform induces homeomorphisms

$$
\operatorname{Inf}_{\text {odd }}(H) \stackrel{c}{\approx} \mathrm{O}\left(H_{\mathbb{R}}\right) \cap(\mathcal{K}+\mathbb{C} \cdot \mathbb{1}) \text { and } \operatorname{Inf}_{\text {odd }}^{\text {fin }}(H) \stackrel{c}{\approx} \mathrm{O}\left(H_{\mathbb{R}}\right) \cap(\mathcal{F R}+\mathbb{C} \cdot \mathbb{1})
$$

Here $\operatorname{Inf}_{\text {odd }}(H)$ denotes the subspace of odd operators in $\operatorname{Inf}(H)$ (which are still $\mathbb{C}$-linear $)$ and $\mathrm{O}\left(H_{\mathbb{R}}\right)$ is the usual orthogonal group, thought of as the subgroup of real operators in the unitary group $\mathrm{U}(H)$.

Proof. Since both sides have the subspace topology, it suffices to show that the Cayley transform is a bijection between the spaces given in the Proposition. An operator $D$ in $\operatorname{Inf}(H)$ is odd if and only if $D^{\alpha}:=\alpha D \alpha=-D$. Since our grading involution $\alpha$ is $\mathbb{C}$-antilinear, we also have $i^{\alpha}=-i$ and therefore ${ }^{1}$

$$
\overline{c(D)}=c(D)^{\alpha}=\left(\frac{D+i}{D-i}\right)^{\alpha}=\frac{-D-i}{-D+i}=\frac{D+i}{D-i}=c(D)
$$

Since the operators in $\mathcal{U}(H)$ that commute with complex conjugation are clearly those in the real orthogonal group $\mathrm{O}\left(H_{\mathbb{R}}\right)$, we get $c(D) \in \mathrm{O}\left(H_{\mathbb{R}}\right)$ and as before, $c(D)-\mathbb{1}$ is compact (respectively finite rank). Conversely, a similar calculation shows that if $c(D)$ is real then $D^{\alpha}=-D$.

Let $\mathcal{H}_{n}$ be as in the introduction, a graded real Hilbert space with a $*$-action of the real Clifford algebra $C_{n}$. For example, the above discussion is the case $n=1$ if we define $\mathcal{H}_{1}=H$ with the grading and $C_{1}=\mathbb{C}$-action as above. Note that in this case one can think of $\mathbb{C}$-linear operators, say in $\mathcal{K}(H)$, as $\mathbb{R}$-linear operators that commute with the $C_{1}$-action. This motivates the following definition.

\footnotetext{
${ }^{1}$ The notation $(D \pm i)^{-1}$ best interpreted on each pair of eigenspaces $V_{\lambda} \oplus V_{-\lambda}$ of $D$ separately; there it definitely makes sense and that is all we care about here.
} 
Definition 3.8. We denote by $\mathcal{K}_{n}$ (respectively $\mathcal{F R}_{n}$ ) the space of all $C_{n^{-}}$ linear self-adjoint compact (respectively finite rank) operators on $\mathcal{H}_{n}$, and by $\operatorname{Inf}_{n}$ (respectively $\operatorname{Inf}_{n}^{\text {fin }}$ ) the subspace of Inf (respectively $\operatorname{Inf}^{\text {fin }}$ ) that consists of all $C_{n}$-linear and odd operators.

In order to extend Proposition 3.7 to $\operatorname{Inf}_{n}$ we have to identify the image of these spaces under the Cayley transform in $\mathrm{O}\left(H_{n}\right)$. We now assume the following model for our graded Hilbert space $\mathcal{H}_{n}$. For $n \geq 1$, let $H_{n}$ be a real Hilbert space that is a $C_{n}^{\text {ev }}$-module and consider the graded $C_{n}$-module

$$
\mathcal{H}_{n}:=H_{n} \otimes_{C_{n}^{\text {ev }}} C_{n} \cong H_{n} \otimes_{\mathbb{R}} \mathbb{C} .
$$

The last isomorphism should be interpreted as saying that the complex structure on $\mathcal{H}_{n}$ is given by the last basis element $e_{n} \in C_{n}$ and that the grading can be thought of as corresponding to complex conjugation, just like in our previous discussion.

Proposition 3.9. For all $n \geq 1$ there are homeomorphisms

$$
\operatorname{Inf}_{n}^{\text {fin }} \approx \mathcal{M}_{n}^{\text {fin }} \text { and } \quad \operatorname{Inf}_{n} \approx \mathcal{M}_{n}
$$

For $n=1$ they are given by the Cayley transform $D \mapsto c(D)$ and for $n>1$ by $D \mapsto e_{n-1} c(D)$.

Proof. We will show that there is a homeomorphism $\operatorname{Inf}_{n} \approx \mathcal{M}_{n}$ which restricts to the desired homeomorphism on $\operatorname{Inf}_{n}^{\mathrm{fin}}$. The case $n=1$ was discussed above because in this case we have by definition

$$
\mathrm{O}\left(H_{1}\right) \cap(\mathbb{1}+\mathcal{K})=\mathcal{M}_{1} \quad \text { and } \quad \mathrm{O}\left(H_{1}\right) \cap(\mathbb{1}+\mathcal{F R})=\mathcal{M}_{1}^{\mathrm{fin}} .
$$

Now, let $n \geq 2$. Recall that the complex structure on $\mathcal{H}_{n}$ is given by $e_{n}$, hence the relation $D e_{n}=e_{n} D$ gives the $\mathbb{C}$-linearity of $c(D)$. We claim that the relations $D e_{i}=e_{i} D$ for the remaining $n-1$ generators $e_{i}$ of $C_{n}$ imply that the generators $e_{i}$ of $C_{n-1}$ satisfy

$$
e_{i} c(D)=c(D)^{-1} e_{i}
$$

To see this, note that we have the relations

$$
e_{i}(D \pm i)=(D \mp i) e_{i} \quad \text { and } \quad e_{i}(D \pm i)^{-1}=(D \mp i)^{-1} e_{i}
$$

which together yield

$$
e_{i} c(D)=e_{i}(D-i)(D+i)^{-1}=(D+i)(D-i)^{-1} e_{i}=c(D)^{-1} e_{i} .
$$

Note that all these are operators on $\mathcal{H}_{n}$ but that our odd operator $D$ gives an action of $c(D)$ on $H_{n}$. We assert that the same relation holds for this operator $c(D)$ on $H_{n}$. First, note that since $c(D)$ is $\mathbb{C}$-linear, i.e., it commutes with $e_{n}$, we have $e_{n} e_{i} c(D)=c(D)^{-1} e_{n} e_{i}$. Next, one checks that under the isomorphism $C_{n}^{\mathrm{ev}} \cong C_{n-1}$ the action of $e_{n} e_{i} \in C_{n}, i=1, \ldots, n-1$, corresponds to the automorphism $e_{i} \otimes$ id of $H_{n} \otimes \mathbb{C}$. This together with the relation we computed for $c(D) \otimes$ id implies $e_{i} c(D)=c(D)^{-1} e_{i}$ for $i=1, \ldots, n-1$.

Hence we see that the Cayley transform $c$ gives a homeomorphism

$$
\operatorname{Inf}_{n} \approx\left\{A \in \mathrm{O}\left(H_{n}\right) \mid A \equiv \mathbf{1} \bmod \mathcal{K}\left(H_{n}\right) \text { and } e_{i} A=A^{-1} e_{i} \text { for } i=1, \ldots, n-1\right\} .
$$

The space on the right-hand side is not quite $\mathcal{M}_{n}$ yet. However, we claim that it can be identified with $\mathcal{M}_{n}$ by associating to an operator $A$ the complex structure

$$
J:=e_{n-1} A \in \mathcal{M}_{n} .
$$


It is clear that $J \equiv e_{n-1} \bmod \mathcal{K}\left(H_{n}\right)$. Furthermore, $J$ is indeed a complex structure:

$$
J^{2}=e_{n-1} A e_{n-1} A=e_{n-1} A A^{-1} e_{n-1}=-\mathbb{1} .
$$

It remains to check that $J$ anticommutes with the generators of $C_{n-2}$. The following computation shows this claim using $\tilde{e}_{i}=e_{n-1} e_{i}$ :

$$
\left(e_{n-1} e_{i}\right)\left(e_{n-1} A\right)=\left(e_{n-1} e_{i}\right)\left(A^{-1} e_{n-1}\right)=e_{n-1} A e_{i} e_{n-1}=-\left(e_{n-1} A\right)\left(e_{n-1} e_{i}\right)
$$

where we have interpreted $H_{n}$ as a module over $C_{n-2}$ via

$$
C_{n-2} \stackrel{\cong}{\rightarrow} C_{n-1}^{\mathrm{ev}}, \quad \tilde{e}_{i} \mapsto e_{n-1} e_{i},
$$

with $\tilde{e}_{1}, \ldots, \tilde{e}_{n-2}$ denoting the standard generators of $C_{n-2}$.

For later use, we define the real graded $C^{*}$-algebra $\mathcal{S}$ to be given by real-valued functions in $C_{0}(\mathbb{R})$ with trival $*$ and with grading involution induced by $x \mapsto-x$ (leading to the usual decomposition into even and odd functions).

To motivate the use of self-adjoint operators, we make the following easy observation that comes from the above case $n=1$.

Lemma 3.10. Restriction to self-adjoint elements defines homeomorphisms

$$
\operatorname{Hom}_{\text {gr }}\left(C_{0}(\mathbb{R}), \mathcal{K}(H)\right) \leftrightarrow \operatorname{Hom}_{\text {gr }}\left(\mathcal{S}, \mathcal{K}^{s a}(H)\right)
$$

where $H$ is a complex Hilbert space with grading involution as above and $\mathrm{Hom}_{\mathrm{gr}}$ denotes grading preserving $*$-homomorphisms. The analogous statement holds for $\mathcal{F} \mathcal{R}$ in place of $\mathcal{K}$.

Proof. Recall that $\mathcal{S}$ are just the real valued functions in $C_{0}(\mathbb{R})$ (also known as the self-adjoint elements in this complex $C^{*}$-algebra) and that the grading involutions agree. Moreover, there is an isomorphism

$$
\mathcal{S} \otimes_{\mathbb{R}} \mathbb{C} \cong C_{0}(\mathbb{R})
$$

The same statements apply to $\mathcal{K}$ (respectively $\mathcal{F} \mathcal{R}$ ) and therefore the complexification map gives an inverse to the restriction map in the lemma.

The following result follows from the observation that for an odd operator $D \in$ Inf, functional calculus leads to a grading preserving $*$-homomorphism $f \mapsto f(D)$ from $\mathcal{S}$ to $\mathcal{K}$. Vice versa, if this $*$-homomorphism is grading preserving then $D$ must have been odd to start with.

Proposition 3.11. Functional calculus induces the homeomorphisms

$$
\operatorname{Inf}_{n} \approx \operatorname{Hom}_{\mathrm{gr}}\left(\mathcal{S}, \mathcal{K}_{n}\right) \quad \text { and } \quad \operatorname{Inf}_{n}^{\mathrm{fin}} \approx \operatorname{Hom}_{\mathrm{gr}}\left(\mathcal{S}, \mathcal{F R}_{n}\right)
$$

\section{Configuration spaces}

The unbounded operators of the previous section can be visualized as configurations on the real line: an operator $D \in \operatorname{Inf}$ is completely determined by its eigenvalues and eigenspaces and hence by the map $V$ that associates to $\lambda \in \mathbb{R}$ the subspace $V(\lambda)$ on which $D=\lambda$. We call $V$ a 'configuration on $\mathbb{R}^{\text {', since } V(\lambda)}$ may be thought of as a label attached at $\lambda \in \mathbb{R}$. Since slightly different spaces of configurations will appear in Section 8 , we give a general definition that also covers the case considered there.

Let $\Lambda$ be a topological space equipped with an involution $s$ and $\mathcal{H}$ a separable graded Hilbert space with grading involution $\alpha$. A configuration on $\Lambda$ indexed 
by orthogonal subspaces of $\mathcal{H}$ is a map $V$ from $\Lambda$ to the set of closed (ungraded) subspaces of $\mathcal{H}$ such that

- the subspaces $V(\lambda)$ are pairwise orthogonal

- $\mathcal{H}$ is the Hilbert sum of the $V(\lambda)$ 's

- $V$ is compatible with $s$ and $\alpha$, i.e., $V(s(\lambda))=\alpha(V(\lambda))$ for all $\lambda \in \Lambda$.

Recall that closed subspaces of $\mathcal{H}$ correspond precisely to continuous self-adjoint projection operators on $\mathcal{H}$. Hence we may interpret $V$ as a map

$$
V: X \rightarrow \operatorname{Proj}(\mathcal{H}) \subset B(\mathcal{H}) \text {. }
$$

To save space, we write $V_{\lambda}:=V(\lambda)$. Define $\operatorname{supp}(V):=\overline{\left\{\lambda \in \Lambda \mid V_{\lambda} \neq 0\right\}}$.

Definition 4.1. The space $\operatorname{Conf}(\Lambda ; \mathcal{H})$ of configurations on $\Lambda$ indexed by orthogonal subspaces of $\mathcal{H}$ is the set of all configurations $V: \Lambda \rightarrow \operatorname{Proj}(\mathcal{H})$ equipped with the topology generated by the subbasis consisting of the sets

$$
\mathcal{B}(U, L):=\left\{V \in \operatorname{Conf}(\Lambda ; \mathcal{H}) \mid V_{U}:=\sum_{\lambda \in U} V_{\lambda} \in L, \operatorname{supp}(V) \cap \partial U=\varnothing\right\},
$$

where $U$ and $L$ range over all open subsets $U \subset \Lambda$ and $L \subset \operatorname{Proj}(\mathcal{H})$.

We will need the following variations. Let $\Theta \subset \Lambda$ be a subspace that is preserved under the involution $s$. Define $\operatorname{Conf}(\Lambda, \Theta ; \mathcal{H}) \subset \operatorname{Conf}(\Lambda ; \mathcal{H})$ to be the subspace of configurations $V$ such that $V_{\lambda}$ has finite rank for all $\lambda \in \Theta^{c}:=\Lambda \backslash \Theta$ and such that the subset of all $\lambda \in \Theta^{\mathrm{c}}$ with $V(\lambda) \neq 0$ is discrete in $\Theta^{\mathrm{c}}{ }^{2}$ Replacing the discreteness condition by requiring that there should be only finitely many $\lambda \in \Theta^{\mathrm{c}}$ with $V_{\lambda} \neq 0$ we obtain the space $\operatorname{Conf}^{\text {fin }}(\Lambda, \Theta ; \mathcal{H})$ of configurations that are 'finite away from $\Theta^{\prime}$. Finally, if $C$ is an $\mathbb{R}$-algebra and $\mathcal{H}$ is a $C$-module, we can replace subspaces of $\mathcal{H}$ by $C$-submodules in order to obtain spaces $\operatorname{Conf}_{C}(\Lambda, \Theta ; \mathcal{H})$. If $C$ is graded then we assume that $\mathcal{H}$ is a graded $C$-module, but the subspaces $V(\lambda)$ are still ungraded; only those for which $s(\lambda)=\lambda$ are graded modules over $C$. Our main examples will be the Clifford algebras $C=C_{n}$.

Examples 4.2. Consider the one-point compactification $\overline{\mathbb{R}}$ of $\mathbb{R}$ equipped with the involution $s(x):=-x$. Define

$$
\operatorname{Conf}_{n}:=\operatorname{Conf}_{C_{n}}\left(\overline{\mathbb{R}},\{\infty\} ; \mathcal{H}_{n}\right),
$$

where $\mathcal{H}_{n}$ is the graded $C_{n}$-module from the previous section. We will see in Proposition 4.4 that $\operatorname{Conf}_{n}$ gives a different model for the space $\operatorname{Inf}_{n}$ of unbounded operators introduced above. The homeomorphism $\operatorname{Inf}_{n} \rightarrow \operatorname{Conf}_{n}$ is given by mapping $\mathcal{D} \in \operatorname{Inf}_{n}$ to the configuration defined by associating to $\lambda \in \mathbb{R}$ the $\lambda$-eigenspace $V_{\lambda}$ of $\mathcal{D}$. Here we let $V_{\infty}:=\operatorname{dom}(\mathcal{D})^{\perp}$. Since $\mathcal{D}$ has compact resolvent, the set of $\lambda \in \mathbb{R}$ with $V_{\lambda} \neq 0$ is indeed discrete in $\mathbb{R}$ and each eigenspace $V_{\lambda}, \lambda \in \mathbb{R}$, is finite-dimensional. The relation $V(s(\lambda))=\alpha(V(\lambda))$ corresponds to $\mathcal{D}$ being odd.

In order to get a better feeling for the topology on $\operatorname{Conf}_{n}$, let us describe a neighborhood basis for each configuration in $\operatorname{Conf}_{n}$. This will also be useful for the proof that the map $\operatorname{Inf}_{n} \rightarrow \operatorname{Conf}_{n}$ is a homeomorphism.

We begin by pointing out that the topology on $\operatorname{Conf}_{n}$ is generated by the sets $\mathcal{B}(U, L)$, where $U \subset \mathbb{R}$ is bounded. To see this, note that, by definition of $\operatorname{Conf}_{n}$, $\infty \in \operatorname{supp}(V)$ for all $V \in \operatorname{Conf}_{n}$. Hence $\mathcal{B}(U, L)=\varnothing$ whenever $\infty \in \partial U$ so that

\footnotetext{
${ }^{2}$ This terminology will be convenient for our purposes. However, we should point out that with our notation $\operatorname{Conf}(\Lambda ; \mathcal{H})=\operatorname{Conf}(\Lambda, \Lambda ; \mathcal{H})$ and $\operatorname{not} \operatorname{Conf}(\Lambda ; \mathcal{H})=\operatorname{Conf}(\Lambda, \varnothing ; \mathcal{H})$.
} 
the case of unbounded $U \subset \mathbb{R}$ is irrelevant. Furthermore, if $\infty \in U$ we can use $\mathcal{B}(U, L)=\mathcal{B}\left(U^{\mathrm{c}}, 1-L\right)$ to describe $\mathcal{B}(U, L)$ in terms of $U^{\mathrm{c}}:=\overline{\mathbb{R}} \backslash U$. Thus it is sufficient to consider $\mathcal{B}(U, L)$ for $U \subset \mathbb{R}$ bounded.

Definition 4.3. Let $V \in \operatorname{Conf}_{n}$ and let $K$ be a (large) positive real number such that $V_{K}=0$. Let $B_{K}(0)$ be the ball of radius $K$ around 0 and denote by $\lambda_{1}, \ldots, \lambda_{l_{K}}$ the numbers in $B_{K}(0)$ such that $V_{\lambda_{i}} \neq 0$. Let $\delta>0$ and $\varepsilon>0$ be (small) real numbers; we may choose $\delta$ so small that $B_{\delta}\left(\lambda_{i}\right) \cap B_{\delta}\left(\lambda_{j}\right)=\varnothing$ for $i \neq j$. Denote by $V_{K, \delta, \varepsilon}$ the set of all configurations $W$ such that $\left\|V_{B_{\delta}\left(\lambda_{i}\right)}-W_{B_{\delta}\left(\lambda_{i}\right)}\right\|<\varepsilon$ for all $i$ and such that $W_{\lambda}=0$ for all $\lambda \in B_{K}(0)$ that do not lie in one of the balls $B_{\delta}\left(\lambda_{i}\right)$.

Thus, an element $W \in V_{K, \delta, \varepsilon}$ almost looks like $V$ on $B_{K}(0)$ : the only thing that can happen is that a label $V_{\lambda}$ 'splits' into labels $W_{\lambda_{j}}$ with $\left|\lambda-\lambda_{j}\right|$ small $(<\delta)$ and $\sum_{j} W_{\lambda_{j}}$ close to $V_{\lambda}(<\varepsilon)$. The $V_{K, \delta, \varepsilon}$ indeed form a neighborhood basis of $V$ : assume $V \in \bigcap_{k=1}^{n} \mathcal{B}\left(U_{k}, L_{k}\right)$, with $U_{k} \subset \mathbb{R}$ bounded. Choose $K$ as above with $\bigcup_{k=1}^{n} U_{k} \subset B_{K}(0)$. Picking $\delta>0$ so small that $B_{\delta}\left(\lambda_{i}\right) \subset \bigcap_{k=1}^{n} U_{k}$ for all $i$ it follows easily using the triangle inequality that for $\varepsilon>0$ sufficiently small $V_{K, \delta, \varepsilon} \subset \bigcap_{i=1}^{n} \mathcal{B}\left(U_{i}, L_{i}\right)$.

In particular, we see that the topology on $\operatorname{Conf}_{n}$ controls configurations well on compact subsets of $\mathbb{R}$ but not near infinity. The discussion also shows that Conf ${ }_{n}$ is first countable since we may choose $K, \delta$, and $\varepsilon$ in $\mathbb{Q}$.

Given any $V \in \operatorname{Conf}_{n}=\operatorname{Conf}_{C_{n}}\left(\overline{\mathbb{R}},\{\infty\} ; \mathcal{H}_{n}\right)$ and any function $f \in \mathcal{S}$, we can define a $C_{n}$-linear operator $f(V)$ on $\mathcal{H}_{n}$ by requiring that $f(V)$ has eigenvalue $f(\lambda)$ exactly on $V_{\lambda}$. This operator is always compact and it is of finite rank if and only if $V \in \operatorname{Conf}_{n}^{\text {fin }}:=\operatorname{Conf}_{C_{n}}^{\text {fin }}\left(\overline{\mathbb{R}},\{\infty\} ; \mathcal{H}_{n}\right) \subset \operatorname{Conf}_{n}$, the subspace of configurations that are finite away from $\{\infty\}$. Moreover, the relation $V(s(\lambda))=\alpha(V(\lambda))$ corresponds to $f \mapsto f(V)$ being grading preserving.

Proposition 4.4. Functional calculus $F(V)(f):=f(V)$ gives homeomorphisms

$$
F: \operatorname{Conf}_{n} \stackrel{\approx}{\rightarrow} \operatorname{Hom}_{\mathrm{gr}}\left(\mathcal{S}, \mathcal{K}_{n}\right) \text { and } \operatorname{Conf}_{n}^{\mathrm{fin}} \stackrel{\approx}{\rightarrow} \operatorname{Hom}_{\mathrm{gr}}\left(\mathcal{S}, \mathcal{F} \mathcal{R}_{n}\right)
$$

Combining this result with Proposition 3.11 we obtain as a corollary

$$
\operatorname{Inf}_{n} \approx \operatorname{Conf}_{n} \quad \text { and } \quad \operatorname{Inf}_{n}^{\mathrm{fin}} \approx \operatorname{Conf}_{n}^{\mathrm{fin}}
$$

Proof. It is clear that $F$ is a bijection because the map that identifies operators with the eigenspaces and eigenvalues is obviously a bijection and it is the composition of $F$ with a homeomorphism. Since Conf $_{n}$ is first countable, we can check the continuity of $F$ on sequences. To do so, assume $V_{n} \rightarrow V$ and fix $f \in \mathcal{S}$. We have to prove $f\left(V_{n}\right) \rightarrow f(V)$. Given $\varepsilon>0$, choose $K>0$ such that $|f(x)|<\varepsilon$ if $|x|>K$. Since the continuous map $f$ is automatically uniformly continuous on compact sets, we can find a $\delta>0$ such that for all $x \in B_{K}(0)$ we have $|f(x)-f(y)|<\varepsilon$ provided $|x-y|<\delta$. The assumption $V_{n} \rightarrow V$ tells us that $V_{n} \in V_{K, \delta, \varepsilon}$ for large $n$. 
The claim now follows from the following estimate that holds for all $W \in V_{K, \delta, \varepsilon}$ :

$$
\begin{aligned}
\|f(V)-f(W)\| & =\left\|\sum_{\lambda \in \mathbb{R}} f(\lambda) V_{\lambda}-\sum_{\mu \in \mathbb{R}} f(\mu) W_{\mu}\right\| \\
& \leq\left\|\sum_{\substack{\lambda \in B_{K}(0) \\
V_{\lambda} \neq 0}}\left(f(\lambda) V_{\lambda}-\sum_{\mu \in B_{\delta}(\lambda)} f(\mu) W_{\mu}\right)\right\|+2 \varepsilon \\
& \leq \#\left\{\lambda \in B_{K}(0) \mid V_{\lambda} \neq 0\right\} \cdot\left(\max _{\lambda \in B_{K}(0)} f(\lambda) \cdot \varepsilon+\varepsilon\right)+2 \varepsilon \\
& \leq C \cdot \varepsilon,
\end{aligned}
$$

where the constant $C$ only depends on $f$ and $V$. The first inequality follows by rearranging the terms and using the triangle inequality together with $|f(x)|<\varepsilon$ for $|x|>K$. The second inequality follows from

$$
\begin{aligned}
\| f(\lambda) V_{\lambda}-\sum_{\mu \in B_{\delta}(\lambda)} f(\mu) & W_{\mu} \| \\
& \leq\left\|f(\lambda)\left(V_{\lambda}-W_{B_{\delta}(\lambda)}\right)\right\|+\left\|\sum_{\mu \in B_{\delta}(\lambda)}(f(\lambda)-f(\mu)) W_{\mu}\right\| \\
& \leq \max _{\lambda \in B_{K}(0)} f(\lambda) \cdot \varepsilon+\varepsilon .
\end{aligned}
$$

The space $\operatorname{Hom}_{\mathrm{gr}}\left(S, \mathcal{K}_{n}\right)$ is also first countable. This follows since $f\left(V_{n}\right) \rightarrow$ $f(V)$ for all $f \in \mathcal{S}$ if and only if this is the case for $f(x)=\mathrm{e}^{-x^{2}}$ and $f(x)=x \mathrm{e}^{-x^{2}}$ (see the proof of Lemma 5.12). Thus we can check the continuity of $F^{-1}$ on sequences as well. Assume $f\left(V_{n}\right) \rightarrow f(V)$ for all $f \in \mathcal{S}$ and $V \in \mathcal{B}(U, L)$. We have to show $V_{n} \in \mathcal{B}(U, L)$ for $n$ sufficiently large. More explicitly: $\operatorname{supp}\left(V_{n}\right) \cap \partial U=\varnothing$ for $n$ large and $\lim _{n \rightarrow \infty}\left\|\left(V_{n}\right)_{U}-V_{U}\right\|=0$.

Note that for an accumulation point $\gamma \in \mathbb{R}$ of the set $\bigcup_{n} \operatorname{supp}\left(V_{n}\right)$ we must have $V_{\gamma} \neq 0$, because otherwise we would also have $\left\|f\left(V_{n}\right)-f(V)\right\| \geq \frac{1}{2}$ for infinitely many $n$ if we choose $f$ to be a bump function with $f(\gamma)=1$ that is concentrated near $\gamma$. This together with $\operatorname{supp}(V) \cap \partial U=\varnothing$ implies that there is a neighborhood $v(\partial U)$ of $\partial U$ such that $\left(V_{n}\right)_{\lambda} \neq 0$ for $\lambda \in v(\partial U)$ occurs only for finitely many $n$. In particular, $\operatorname{supp}\left(V_{n}\right) \cap \partial U=\varnothing$ for $n$ large. Now, choose $f \in \mathcal{S}$ such that $\left.f\right|_{\mathbb{R} \backslash U}=0$ and $\left.f\right|_{U \backslash v(\partial U)}=1$. By construction, $f\left(V_{n}\right)=\chi_{U}\left(V_{n}\right)$ for $n$ large, where $\chi_{U}$ denotes the indicator function for $U$. The same identity holds for $V$ and hence we can conclude

$$
\lim _{n \rightarrow \infty}\left\|\left(V_{n}\right)_{U}-V_{U}\right\|=\lim _{n \rightarrow \infty}\left\|\chi_{U}\left(V_{n}\right)-\chi_{U}(V)\right\|=\lim _{n \rightarrow \infty}\left\|f\left(V_{n}\right)-f(V)\right\|=0 .
$$

This completes the proof.

Remark 4.5. A continuous map $f: \Lambda \rightarrow \Lambda^{\prime}$ that commutes with the involutions on $\Lambda$ and $\Lambda^{\prime}$ induces

$$
f_{*}: \operatorname{Conf}(\Lambda ; \mathcal{H}) \rightarrow \operatorname{Conf}\left(\Lambda^{\prime} ; \mathcal{H}\right), \quad\left(f_{*}(V)\right)_{\lambda^{\prime}}:=\sum_{\lambda \in f^{-1}\left(\lambda^{\prime}\right)} V_{\lambda} .
$$

We show that the map $f_{*}$ is continuous under the assumption that the space $\Lambda^{\prime}$ is normal. Let $V \in f_{*}^{-1}(\mathcal{B}(U, L))$. From the definition of $\mathcal{B}(U, L)$ we find $\operatorname{supp}\left(f_{*} V\right) \cap$ 
$\partial U=\varnothing$. Since $\Lambda^{\prime}$ is normal, there is an open neighborhood $N_{V}$ of $\operatorname{supp}\left(f_{*} V\right)$ such that $\overline{N_{V}} \cap \partial U=\varnothing$. Unravelling the definitions one finds

$$
V \in \mathcal{B}\left(f^{-1}(U), L\right) \cap \mathcal{B}\left(f^{-1}\left(N_{V}\right),\left\{\operatorname{id}_{\mathcal{H}}\right\}\right) \subset f_{*}^{-1}(\mathcal{B}(U, L))
$$

so that $f_{*}^{-1}(\mathcal{B}(U, L))$ is a neighborhood of $V$. Thus $f_{*}^{-1}(\mathcal{B}(U, L))$ is open.

The additional properties that we required in the definition of the spaces $\operatorname{Conf}(\Lambda, \Theta ; \mathcal{H})$ are not stable under pushforward. In order to get an induced map we have to require $f$ to be 'nice'. For example, if $f:(\Lambda, \Theta) \rightarrow\left(\Lambda,{ }^{\prime} \Theta^{\prime}\right)$ is a proper map between locally compact Hausdorff spaces, we get an induced map $f_{*}: \operatorname{Conf}(\Lambda, \Theta ; \mathcal{H}) \rightarrow \operatorname{Conf}\left(\Lambda,{ }^{\prime} \Theta^{\prime} ; \mathcal{H}\right)$.

A sightly more complicated argument along the same lines can be used to show that a homotopy $h: \Lambda \times[0,1] \rightarrow \Lambda^{\prime}$ induces a homotopy $H: \operatorname{Conf}(\Lambda, \mathcal{H}) \times[0,1] \rightarrow$ $\operatorname{Conf}\left(\Lambda,{ }^{\prime} \mathcal{H}\right)$, at least if $\Lambda$ is compact.

The following result implies that the 'finite' and 'nonfinite' versions of the spaces considered in the previous chapters are homotopy equivalent.

Proposition 4.6. The inclusion $\mathrm{Conf}_{n}^{\mathrm{fin}} \hookrightarrow \mathrm{Conf}_{n}$ is a homotopy equivalence.

Proof. Consider the family of maps $h_{t}: \overline{\mathbb{R}} \rightarrow \overline{\mathbb{R}}$ defined by

$$
h_{t}(x):= \begin{cases}x /(1-t|x|) & \text { if } x \in(-1 / t, 1 / t) \\ \infty & \text { else. }\end{cases}
$$

These induce a homotopy

$$
H_{t}:=\left(h_{t}\right)_{*}: \operatorname{Conf}_{n} \rightarrow \operatorname{Conf}_{n}
$$

from the identity on $\operatorname{Conf}_{n}$ to $H_{1}$. Note that the image of $H_{1}$ equals Conf fin ${ }_{n}$. Thus, we see that the inclusion $\operatorname{Conf}_{n}^{\text {fin }} \hookrightarrow \operatorname{Conf}_{n}$ is a homotopy equivalence with homotopy inverse $H_{1}: \operatorname{Conf}_{n} \rightarrow \operatorname{Conf}_{n}^{\text {fin }}$.

Remark 4.7. We will later consider the space $\operatorname{colim}_{k \rightarrow \infty} \operatorname{Conf}_{n}^{(k)}$, where $\operatorname{Conf}_{n}^{(k)}$ is the subspace of configurations $V \in \operatorname{Conf}_{n}$ such that $V_{\lambda} \neq 0$ for at most $k$ numbers $\lambda$ with $0<\lambda<\infty$. As a set, $\operatorname{colim}_{k \rightarrow \infty} \operatorname{Conf}_{n}^{(k)}$ is just $\operatorname{Conf}_{n}^{\text {fin }}$, but the topology has more open sets than the topology of Conf $f_{n}^{\text {fin }}$. We claim that

$$
\underset{k \rightarrow \infty}{\operatorname{colim}} \operatorname{Conf}_{n}^{(k)} \hookrightarrow \operatorname{Conf}_{n}
$$

is also a homotopy equivalence. The same homotopy as in the proof of Proposition 4.6 can be used. This works because the map $H_{1}: \operatorname{Conf}_{n} \rightarrow \operatorname{colim}_{k \rightarrow \infty} \operatorname{Conf}_{n}^{(k)}$ is still continuous. This follows from the observation that

$$
C: \operatorname{Conf}_{n} \rightarrow \mathbb{N}, \quad V \mapsto C(V):=\sum_{-1 \leq \lambda \leq 1} \operatorname{dim} V_{\lambda}
$$

is locally bounded so that for every $V \in \operatorname{Conf}_{n}$ we can find an open neighborhood $N$ of $V$ such that $H_{1}(N) \subset \operatorname{Conf}_{n}^{(k)}$ for some $k$ (since $H_{1}$ moves all labels outside $(-1,1)$ to $\infty)$. Since continuity can be checked locally and since on $\operatorname{Conf}_{n}^{(k)}$ the topology induced from $\operatorname{Conf}_{n}$ and the colimit topology coincide, it follows that $H_{1}: \operatorname{Conf}_{n} \rightarrow \operatorname{colim}_{k \rightarrow \infty} \operatorname{Conf}_{n}^{(k)}$ is continuous.

In particular, we see that the identity map id: $\operatorname{colim}_{k \rightarrow \infty} \operatorname{Conf}_{n}^{(k)} \rightarrow \operatorname{Conf}_{n}^{\text {fin }}$ is a homotopy equivalence. 
The same argument applies in the case of the filtration $X_{k}$ of Conf ${ }_{n}^{\text {fin }}$ given by the dimension of a configuration: if $X_{k} \subset \mathrm{Conf}_{n}^{\mathrm{fin}}$ is the subspace of configurations $V$ with $\operatorname{dim}(V):=\operatorname{dim}_{C_{n}}\left(\bigoplus_{\lambda \in \mathbb{R}} V_{\lambda}\right) \leq 2 k$, then the identity map $\operatorname{colim}_{k \rightarrow \infty} X_{k} \rightarrow$ $\mathrm{Conf}_{n}^{\text {fin }}$ is a homotopy equivalence.

\section{Super-semigroups of operators}

In this section we will define super-semigroups of operators (SGOs) using as little supermathematics as possible. We will only need basic definitions and results from the theory of super manifolds, as can be found in Chapter 2 of [6]. Supermanifolds are particular ringed spaces, i.e., topological spaces together with a sheaf of rings, and morphisms are maps of ringed spaces. The local model for a super manifold of dimension $(p \mid q)$ is Euclidean space $\mathbb{R}^{p}$ equipped with the sheaf of commutative super $\mathbb{R}$-algebras

$$
U \mapsto C^{\infty}(U) \otimes \Lambda^{*}\left(\mathbb{R}^{q}\right) .
$$

This ringed space is the super manifold $\mathbb{R}^{p \mid q}$.

Definition 5.1. A super manifold $M$ of dimension $(p \mid q)$ is a pair $\left(|M|, \mathcal{O}_{M}\right)$ consisting of a (Hausdorff and second countable) topological space $|M|$ together with a sheaf of commutative super $\mathbb{R}$-algebras $\mathcal{O}_{M}$ that is locally isomorphic to $\mathbb{R}^{p \mid q}$.

To every super manifold $M$ there is an associated reduced manifold

$$
M^{\mathrm{red}}:=\left(|M|, \mathcal{O}_{M} / \text { nil }\right)
$$

obtained by dividing out nilpotent functions. By construction, this gives a smooth manifold structure on the underlying topological space $|M|$ and there is an inclusion of super manifolds $M^{\text {red }} \hookrightarrow M$. For example, $\left(\mathbb{R}^{p \mid q}\right)^{\text {red }}=\mathbb{R}^{p}$.

The main invariant of a super manifold $M$ is its ring of functions $C^{\infty}(M)$, defined as the global sections of the sheaf $\mathcal{O}_{M}$. For example, $C^{\infty}\left(\mathbb{R}^{p \mid q}\right)=C^{\infty}\left(\mathbb{R}^{p}\right) \otimes$ $\Lambda^{*}\left(\mathbb{R}^{q}\right)$. It turns out that the maps between super manifolds $M$ and $N$ are just given by grading preserving algebra homomorphisms between the rings of functions:

$$
\operatorname{Hom}(M, N) \cong \operatorname{Hom}_{\mathrm{Alg}}\left(C^{\infty}(N), C^{\infty}(M)\right)
$$

Example 5.2. Let $E \rightarrow M$ be a real vector bundle of fiber dimension $q$ over the smooth manifold $M^{p}$. Then $\left(M, \Gamma\left(\Lambda^{*} E\right)\right)$ is a super manifold of dimension $(p \mid q)$. Batchelor's theorem [3] says that every super manifold is isomorphic (but not canonically) to one of this type. This result does not hold in analytic categories, and it shows that, in the smooth category, super manifolds are only interesting if one takes their morphisms seriously and doesn't just consider isomorphism classes.

Definition 5.3. Define the 'twisted' super Lie group structure on $\mathbb{R}^{1 \mid 1}$ by

$$
m: \mathbb{R}^{1 \mid 1} \times \mathbb{R}^{1 \mid 1} \rightarrow \mathbb{R}^{1 \mid 1}, \quad(t, \theta),(s, \eta) \mapsto(t+s+\theta \eta, \theta+\eta) .
$$

This super Lie group plays a special role in supergeometry, the reason being the particular structure of its super Lie algebra: $\operatorname{Lie}\left(\mathbb{R}^{1 \mid 1}\right) \cong \mathbb{R}[D]$ is the super Lie algebra generated freely by one odd generator $D$. Thus, $\mathbb{R}^{1 \mid 1}$ may be considered the odd analogue of the Lie group $\mathbb{R}$. For example, integrating an odd vector field on a super manifold $M$ leads to a flow $M \times \mathbb{R}^{1 \mid 1} \rightarrow M$, and formulating the flow property involves the 'twisted' group structure. 
From the definition of $m$ it is clear that the open sub-super manifold $\mathbb{R}_{>0}^{1 \mid 1}$ defined by the inclusion $\mathbb{R}_{>0} \subset \mathbb{R}$ inherits the structure of a super-semigroup. ${ }^{3}$ Now we can already guess what a SGO should be: just as an ordinary semigroup of operators is a homomorphism from $\mathbb{R}_{>0}$ to an algebra of operators, a supersemigroup of operators will be a homomorphism from the super-semigroup $\mathbb{R}_{>0}^{1 \mid 1}$ to a $\left(\mathbb{Z}_{2}\right.$-graded) operator algebra. In order to make sense of such a homomorphism, we will consider the latter to be a generalized super-semigroup using the 'functor of points' formalism (see [6, Sections 2.8-2.9]). Note that we, implicitly, already used the 'functor of points' language when writing down the group law $m$. The formula above tells us what the product of two elements in the $\operatorname{group} \operatorname{Hom}\left(S, \mathbb{R}^{1 \mid 1}\right)$ is. Since the rule holds functorially for all super manifolds $S$, this defines the map $m$ by the Yoneda lemma.

Finally, we would like to remark that the structure of $\operatorname{Lie}\left(\mathbb{R}^{1 \mid 1}\right)$ and the existence of an odd infinitesimal generator $\mathcal{D}$ for a $\mathrm{SGO} \Phi$ that we will prove below are closely related: $\mathcal{D}$ is nothing but the image of $D$ under the derivative of $\Phi$. However, making this precise requires some work (note that $\Phi$ maps to an infinitedimensional space!). We will avoid such problems altogether: the super Lie algebras do not appear in our argument.

5.1. Generalized super manifolds and super Lie groups. We will use the following, somewhat primitive, extension of the notion of super manifolds:

Definition 5.4. A generalized super manifold $M$ is a contravariant functor from super manifolds to sets. ${ }^{4}$ Similarly, if $M$ takes values in the category of (semi)groups, we call it a generalized super-(semi)group. Morphisms in all these categories are natural transformations.

Examples 5.5. (1) The Yoneda lemma implies that super manifolds are embedded as a full subcategory in generalized super manifolds by associating to a super manifold $M$ the functor

$$
S \mapsto M(S):=\operatorname{Hom}(S, M) .
$$

The analogous statement holds for super-(semi)groups. For example, we will consider $\mathbb{R}_{>0}^{1 \mid 1}$ as a generalized super-semigroup by identifying it with the contravariant functor

$$
S \mapsto \operatorname{Hom}\left(S, \mathbb{R}_{>0}^{1 \mid 1}\right)
$$

from super manifolds to semigroups.

(2) Every $\mathbb{Z}_{2}$-graded real Banach space $B=B_{0} \oplus B_{1}$ may be considered as a generalized super manifold as follows. We define the value of the functor $B$ on a superdomain $U=\left(|U|, C^{\infty}\left(_{-}\right)\left[\theta_{1}, \ldots, \theta_{q}\right]\right) \subset \mathbb{R}^{p \mid q}$ to be

$$
B(U):=\left(C^{\infty}(|U|, B)\left[\theta_{1}, \ldots, \theta_{q}\right]\right)^{\mathrm{ev}} .
$$

The superscript ev indicates that we pick out the even elements, so that an element $f \in B(U)$ is of the form

$$
f=\sum_{I} f_{I} \theta^{I}
$$

\footnotetext{
${ }^{3} \mathrm{~A}$ super (Lie) semigroup is a super manifold $M$ together with an associative multiplication $M \times M \rightarrow M$. In terms of the functor of points language: the morphism sets $\operatorname{Hom}(S, M) \operatorname{carry}$ semigroup structures, functorially in $S$.

${ }^{4}$ We use this simple notion here in order to avoid dealing with infinite-dimensional super manifolds.
} 
where $I \subset\{1, \ldots, q\}$ and $\theta^{I}:=\prod_{j \in I} \theta_{j}$ and each $f_{I}$ is a smooth map $|U| \rightarrow B_{|I|}$. For a map $\varphi: U^{\prime} \rightarrow U$ between superdomains, the map $B(\varphi)$ is defined using the formal Taylor expansion, just as in the case of usual super manifolds. This functor on superdomains may be extended to the whole category of super manifolds by gluing.

(3) If $B$ is a $\mathbb{Z}_{2}$-graded Banach algebra, $B(U)$ is an algebra and thus $B$ is a generalized super-semigroup. Again, $B$ may be extended to all super manifolds by gluing.

Remark 5.6. Giving a morphism from an ordinary super manifold $T$ to a generalized super manifold $B$ amounts to prescribing the image of the universal element id $\in \operatorname{Hom}(T, T)$ in $B(T)$. Hence $B(T)$ is exactly the set of morphisms from $T$ to $B$.

Now assume that, in addition, $T$ and $B$ carry super-(semi)group structures. A map $\Phi: T \rightarrow B$ is a homomorphism if

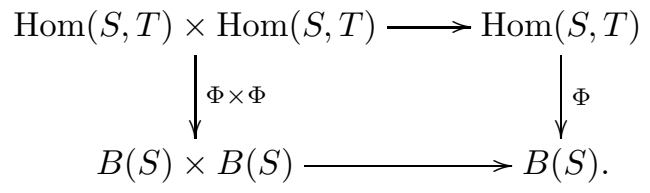

commutes for all super manifolds $S$. Again, it suffices to check the commutativity for the universal element

$$
\operatorname{pr}_{1} \times \operatorname{pr}_{2} \in \operatorname{Hom}(T \times T, T) \times \operatorname{Hom}(T \times T, T) .
$$

Definition 5.7. Let $\mathcal{H}$ be a $\mathbb{Z}_{2}$-graded Hilbert space, and denote by $B(\mathcal{H})$ the Banach algebra of bounded operators on $\mathcal{H}$ equipped with the $\mathbb{Z}_{2}$-grading inherited from $\mathcal{H}$.

(1) A super-semigroup of operators on $\mathcal{H}$ is a morphism of generalized supersemigroups

$$
\Phi: \mathbb{R}_{>0}^{1 \mid 1} \rightarrow B(\mathcal{H})
$$

As explained in the previous remark, $\Phi$ is of the form $A+\theta B$, where

$$
A: \mathbb{R}_{>0} \rightarrow B^{\mathrm{ev}}(\mathcal{H}) \quad \text { and } \quad B: \mathbb{R}_{>0} \rightarrow B^{\text {odd }}(\mathcal{H})
$$

are smooth maps. The homomorphism property amounts to certain relations between $A$ and $B$ (cf. the proof of Proposition 5.9).

(2) If $K \subset B(\mathcal{H})$ is a subset, we say $\Phi$ is a super-semigroup of operators with values in $K$ if the images of $A$ and $B$ are contained in $K$.

(3) If $\mathcal{H}$ is a module over the Clifford algebra $C_{n}$, we say $\Phi$ is $C_{n}$-linear if it takes values in $C_{n}$-linear operators.

Examples 5.8. SGOs arise in a natural way from Dirac operators. We give two examples of that type and then extract their characteristic properties to describe a more general class of examples. The verification of the SGO properties for these more general examples also includes the case of Dirac operators.

(1) Let $\mathcal{D}$ be the Dirac operator on a closed spin manifold $X$. There is a corresponding SGO on the Hilbert space of $L^{2}$-sections of the spinor bundle $S$ over $X$. It is given by the super-semigroup of operators

$$
\mathbb{R}_{>0}^{1 \mid 1} \rightarrow B\left(L^{2}(S)\right), \quad(t, \theta) \mapsto \mathrm{e}^{-t \mathcal{D}^{2}}+\theta \mathcal{D} \mathrm{e}^{-t \mathcal{D}^{2}}\left(=\mathrm{e}^{-t \mathcal{D}^{2}+\theta \mathcal{D}}\right)
$$


and takes values in the compact, self-adjoint operators $K^{\mathrm{sa}}\left(L^{2}(S)\right) \subset B\left(L^{2}(S)\right)$.

(2) If $\operatorname{dim} X=n$, one can consider the $C_{n}$-linear spinor bundle and the associated $C_{n}$-linear Dirac operator (see [17, Chapter $\left.2, \S 7\right]$ ). Using the same formula as in the previous example one obtains a $C_{n}$-linear SGO.

(3) Now, let $\mathcal{H}$ be any $\mathbb{Z}_{2}$-graded Hilbert space. For any closed subspace $V_{\infty} \subset$ $\mathcal{H}$ invariant under the grading involution and any odd, self-adjoint operator $\mathcal{D}$ on $V_{\infty}^{\perp}$ with compact resolvent, there is a unique super-semigroup of self-adjoint, compact operators $\Phi=A+\theta B$ defined (using functional calculus) by

$$
A(t)=\mathrm{e}^{-t \mathcal{D}^{2}} \quad \text { and } \quad B(t)=\mathcal{D e}^{-t \mathcal{D}^{2}} \quad \text { on } V_{\infty}^{\perp}
$$

and $A(t)=B(t)=0$ on $V_{\infty}$. The first thing to check is that the maps $A$ and $B$ are indeed smooth; this follows easily using the fact that the map $\mathbb{R}_{>0} \rightarrow C_{0}(\mathbb{R})$, $t \mapsto \mathrm{e}^{-t x^{2}}$, is smooth. Since $\mathcal{D}$ is self-adjoint, the same holds for $A$ and $B$. Finally, we have to show that $\Phi$ is a homomorphism. Let $t, \theta, s, \eta$ be the usual coordinates on $\mathbb{R}_{>0}^{1 \mid 1} \times \mathbb{R}_{>0}^{1 \mid 1}$. It suffices to consider the universal element $\mathrm{pr}_{1} \times \mathrm{pr}_{2}=(t, \theta) \times(s, \eta)$. The computation, which, of course, heavily uses that odd coordinates $\theta$ and $\eta$ square to zero, goes as follows (cf. [28, p. 38]):

$$
\begin{aligned}
\Phi(t, \theta) \Phi & (s, \eta) \\
& =\left(\mathrm{e}^{-t \mathcal{D}^{2}}+\theta \mathcal{D} \mathrm{e}^{-t \mathcal{D}^{2}}\right)\left(\mathrm{e}^{-s \mathcal{D}^{2}}+\eta \mathcal{D} \mathrm{e}^{-s \mathcal{D}^{2}}\right) \\
& =\mathrm{e}^{-t \mathcal{D}^{2}} \mathrm{e}^{-s \mathcal{D}^{2}}+\mathrm{e}^{-t \mathcal{D}^{2}} \eta \mathcal{D} \mathrm{e}^{-s \mathcal{D}^{2}}+\theta \mathcal{D} \mathrm{e}^{-t \mathcal{D}^{2}} \mathrm{e}^{-s \mathcal{D}^{2}}+\theta \mathcal{D} \mathrm{e}^{-t \mathcal{D}^{2}} \eta \mathcal{D e}^{-s \mathcal{D}^{2}} \\
& =\mathrm{e}^{-(t+s) \mathcal{D}^{2}}+(\theta+\eta) \mathcal{D} \mathrm{e}^{-(t+s) \mathcal{D}^{2}}+\theta \mathcal{D} \eta \mathcal{D} \mathrm{e}^{-(t+s) \mathcal{D}^{2}} \\
& =\left(1-\theta \eta \mathcal{D}^{2}\right) \mathrm{e}^{-(t+s) \mathcal{D}^{2}}+(\theta+\eta) \mathcal{D} \mathrm{e}^{-(t+s) \mathcal{D}^{2}} \\
& =\mathrm{e}^{-(t+s+\theta \eta) \mathcal{D}^{2}}+(\theta+\eta) \mathcal{D} \mathrm{e}^{-(t+s+\theta \eta) \mathcal{D}^{2}} \\
& =\Phi(t+s+\theta \eta, \theta+\eta)
\end{aligned}
$$

The second to last equality uses the typical Taylor expansion in supergeometry.

We call $\mathcal{D}$ the infinitesimal generator of $\Phi$. We will see presently that every super-semigroup of self-adjoint, compact operators has a unique infinitesimal generator and is hence one of our examples. Note that if $V_{\infty}$ is a $C_{n}$-submodule and if $\mathcal{D}$ is $C_{n}$-linear, then $A$ and $B$ will also be $C_{n}$-linear.

Next, we will construct infinitesimal generators for super-semigroups of operators. We restrict ourselves to the compact, self-adjoint case, which makes the proof an easy application of the spectral theorem for compact, self-adjoint operators. However, invoking the usual theory of semigroups of operators it should not be too difficult to prove the result for more general SGOs.

Proposition 5.9. Every super-semigroup $\Phi$ of compact, self-adjoint operators on a $\mathbb{Z}_{2}$-graded Hilbert space $\mathcal{H}$ has a unique infinitesimal generator $\mathcal{D}$ as in Example 3 above and is hence of the form

$$
\Phi(t, \theta)=\mathrm{e}^{-t \mathcal{D}^{2}}+\theta \mathcal{D} \mathrm{e}^{-t \mathcal{D}^{2}}
$$

If $\Phi$ is $C_{n}$-linear, so is $\mathcal{D}$.

We need the following technical lemma: 
Lemma 5.10. Let $A, B: \mathbb{R}_{>0} \rightarrow \mathcal{K}^{s a}(H)$ be smooth families of self-adjoint, compact operators on the Hilbert space $H$, and assume that the following relations hold for all $s, t>0$ :

$$
\begin{aligned}
A(s+t) & =A(s) A(t) \\
B(s+t) & =A(s) B(t)=B(s) A(t) \\
A^{\prime}(s+t) & =-B(s) B(t) .
\end{aligned}
$$

Then $H$ decomposes uniquely into orthogonal subspaces $H_{\lambda}, \lambda \in \overline{\mathbb{R}}:=\mathbb{R} \cup\{\infty\}$, such that on $H_{\lambda}$

$$
A(t)=\mathrm{e}^{-t \lambda^{2}} \quad \text { and } \quad B(t)=\lambda \mathrm{e}^{-t \lambda^{2}}
$$

(where we set $\mathrm{e}^{-\infty}=0, \infty \cdot \mathrm{e}^{-\infty}=0$ ). For $\lambda \in \mathbb{R}$, the dimension of $H_{\lambda}$ is finite. Furthermore, the subset of $\lambda$ in $\mathbb{R}$ with $H_{\lambda} \neq 0$ is discrete.

Proof. The identities (1) - (3) above show that all operators $A(s), B(t)$ commute. We apply the spectral theorem for self-adjoint, compact operators to obtain a decomposition of $H$ into simultaneous eigenspaces $H_{\lambda}$ of the $A(s)$ and $B(t)$; the label $\lambda$ takes values in $\overline{\mathbb{R}}$ and will be explained presently. We define functions $A_{\lambda}, B_{\lambda}: \mathbb{R}_{>0} \rightarrow \mathbb{R}$ by

$$
A(t) x=A_{\lambda}(t) x \quad \text { and } \quad B(t) x=B_{\lambda}(t) x \quad \text { for all } x \in H_{\lambda} .
$$

Clearly, $A_{\lambda}$ and $B_{\lambda}$ are smooth and satisfy the same relations as $A$ and $B$.

From (1) we see that $A_{\lambda}$ is nonnegative, and (3) shows $A_{\lambda}^{\prime} \leq 0$, hence $A_{\lambda}$ is decreasing. On the other hand, (1) implies $A_{\lambda}(1 / n)=\sqrt[n]{A_{\lambda}(1)}$, so that

$$
A_{\lambda}(0):=\lim _{t \rightarrow 0} A_{\lambda}(t) \text { exists and equals } 0 \text { or } 1 .
$$

In the first case we conclude $A_{\lambda} \equiv 0$ and thus also $B_{\lambda} \equiv 0$; the label of the corresponding subspace is $\lambda=\infty$. In the second case, we have $A_{\lambda}(1) \neq 0$ and using (1) again we compute

$$
\begin{aligned}
A_{\lambda}^{\prime}(s) & =\frac{A_{\lambda}(1)}{A_{\lambda}(1)} \lim _{t \rightarrow 0} \frac{A_{\lambda}(s+t)-A_{\lambda}(s)}{t} \\
& =\frac{A_{\lambda}(s)}{A_{\lambda}(1)} \lim _{t \rightarrow 0} \frac{A_{\lambda}(1+t)-A_{\lambda}(1)}{t}=-\lambda^{2} A_{\lambda}(s),
\end{aligned}
$$

where $\lambda^{2}:=-A_{\lambda}^{\prime}(1) / A_{\lambda}(1)$ defines the label $\lambda$ up to choice of a sign. By uniqueness of solutions of ODEs, we must have

$$
A_{\lambda}(t)=\mathrm{e}^{-t \lambda^{2}} .
$$

Finally, (3) gives

$$
B_{\lambda}(t)=\lambda \mathrm{e}^{-t \lambda^{2}}
$$

picking the appropriate sign for $\lambda$.

Proof of Proposition 5.9. Let $\Phi=A+\theta B$ be a super-semigroup of compact, self-adjoint operators. As before, we consider $U=\mathbb{R}_{>0}^{1 \mid 1} \times \mathbb{R}_{>0}^{1 \mid 1}$ with coordinates $t, \theta, s, \eta$. For the universal element $\operatorname{pr}_{1} \times \operatorname{pr}_{2}=(t, \theta) \times(s, \eta)$ the homomorphism property of $\Phi$ gives that

$$
\begin{aligned}
\Phi(t+s+\theta \eta, \theta+\eta) & =A(t+s+\theta \eta)+(\theta+\eta) B(t+s+\theta \eta) \\
& =A(t+s)+A^{\prime}(t+s) \theta \eta+(\theta+\eta)\left(B(t+s)+B^{\prime}(t+s) \theta \eta\right) \\
& =A(t+s)+\theta B(t+s)+\eta B(t+s)+\theta \eta A^{\prime}(t+s)
\end{aligned}
$$

This is a free offprint provided to the author by the publisher. Copyright restrictions may apply. 
equals

$$
\begin{aligned}
\Phi(t, \theta) \Phi(s, \eta) & =(A(t)+\theta B(t))(A(s)+\eta B(s)) \\
& =A(t) A(s)+\theta B(t) A(s)+\eta A(t) B(s)-\theta \eta B(t) B(s) .
\end{aligned}
$$

Comparing the coefficients ${ }^{5}$ yields exactly the relations in Lemma 5.10. Using the corresponding decompostion of $\mathcal{H}$ into subspaces $\mathcal{H}_{\lambda}$ we define the operator $\mathcal{D}$ by letting $\mathcal{D}=\lambda$ on $\mathcal{H}_{\lambda}$. From the construction it is clear that $\mathcal{D}$ is the desired infinitesimal generator. Since $A$ is even and $B$ is odd, it follows that $\mathcal{D}$ is an odd operator. If $\Phi$ is $C_{n}$-linear, so is $\mathcal{D}$.

We can finally give the definition promised in part (5) of our Main Theorem.

Definition 5.11. Let $\mathcal{H}$ be a $\mathbb{Z}_{2}$-graded Hilbert space and $C \subset B(\mathcal{H})$ a subspace of the algebra of bounded operators on $\mathcal{H}$. We denote by $\mathcal{S G O}(C)$ the set of super-semigroups of operators with values in $C$ and in particular

$$
\mathcal{S G O}_{n}:=\mathcal{S} \mathcal{G O}\left(\mathcal{K}_{n}\right) \text { and } \mathcal{S G O}_{n}^{\mathrm{fin}}:=\mathcal{S} \mathcal{G O}\left(\mathcal{F R}_{n}\right)
$$

(the subspaces $\mathcal{K}_{n}$ and $\mathcal{F R}_{n}$ were defined in Definition 3.8). We endow $\mathcal{S} \mathcal{G O}(C)$ with the topology of uniform convergence on compact subsets, i.e.,

$$
\Phi_{n}=A_{n}+\theta B_{n} \rightarrow \Phi=A+\theta B
$$

if and only if for all compact $K \subset \mathbb{R}_{>0}$ we have

$$
A_{n}(t) \rightarrow A(t) \quad \text { and } \quad B_{n}(t) \rightarrow B(t) \quad \text { uniformly on } K
$$

with respect to the operator norm on $B(\mathcal{H})$.

We will now relate the spaces $\mathcal{S G O}_{n}$ with our configuration spaces $\operatorname{Conf}_{n}$. We have a triangle (and an analogous one for finite rank operators)

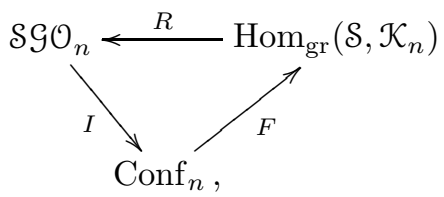

where $I$ maps a super-semigroup of operators to its infinitesimal generator, $F$ is given by functional calculus,

$$
F(\mathcal{D})(f):=f(\mathcal{D})
$$

and $R$ is given by

$$
R(\varphi):=\varphi\left(\mathrm{e}^{-t x^{2}}\right)+\theta \varphi\left(x \mathrm{e}^{-t x^{2}}\right) .
$$

Proposition 5.12. The maps $I, F$, and $R$ are homeomorphisms, and similarly for finite rank operators.

Proof. From the previous discussion it is clear that the composition of the three arrows is the identity no matter where in the triangle we start. We already know from Proposition 4.4 that $F$ is a homeomorphism. We complete the proof by showing that $R$ is a homeomorphism.

\footnotetext{
${ }^{5}$ Just to make the formal aspect of this computation clearer, we would like to point out that the considered identity is an equation in the algebra $K^{\mathrm{sa}}(\mathcal{H})\left(\mathbb{R}_{>0}^{1 \mid 1} \times \mathbb{R}_{>0}^{1 \mid 1}\right)=$ $C^{\infty}\left(\mathbb{R}_{>0} \times \mathbb{R}_{>0}, K^{\mathrm{sa}}(\mathcal{H})\right)[\theta, \eta]^{\mathrm{ev}}$.
} 
The continuity of $R^{-1}$ follows from the following assertion. We claim that we have convergences of operators

$$
f\left(\mathcal{D}_{n}\right) \rightarrow f(\mathcal{D}) \text { for all } f \in C_{0}(\mathbb{R})
$$

if and only if the following two sequences converge:

$$
\mathrm{e}^{-\mathcal{D}_{n}^{2}} \rightarrow \mathrm{e}^{-\mathcal{D}^{2}} \text { and } \quad \mathcal{D}_{n} \mathrm{e}^{-\mathcal{D}_{n}^{2}} \rightarrow \mathcal{D e}^{-\mathcal{D}^{2}}
$$

The first obviously implies the second condition. To see the converse, note that the assumption implies that $f\left(\mathcal{D}_{n}\right) \rightarrow f(\mathcal{D})$ for all $f$ that can be written as a polynomial in the functions $\mathrm{e}^{-x^{2}}$ and $x \mathrm{e}^{-x^{2}}$. Furthermore, the Stone-Weierstraß Theorem implies that $\mathrm{e}^{-x^{2}}$ and $x \mathrm{e}^{-x^{2}}$ generate $C_{0}(\mathbb{R})$ as a $C^{*}$-algebra so that the set of such $f$ is dense in $C_{0}(\mathbb{R})$. Using that from $\|f(\mathcal{D})\| \leq\|f\|$ for all $\mathcal{D}$ and the triangle inequality we can deduce that $f\left(\mathcal{D}_{n}\right) \rightarrow f(\mathcal{D})$ holds for all $f \in C_{0}(\mathbb{R})$.

The continuity of $R$ amounts to showing that if $f\left(\mathcal{D}_{n}\right) \rightarrow f(\mathcal{D})$ for all $f$, then $\mathrm{e}^{-t \mathcal{D}_{n}^{2}} \rightarrow \mathrm{e}^{-t \mathcal{D}^{2}}$ and $\mathcal{D}_{n} \mathrm{e}^{-t \mathcal{D}_{n}^{2}} \rightarrow \mathcal{D e}^{-t \mathcal{D}^{2}}$ uniformly for all $t$ in a compact subset $K \subset \mathbb{R}_{>0}$. As before, we can use $\|f(\mathcal{D})\| \leq\|f\|$ and the triangle inequality to see that for a given $\varepsilon>0$ we can find $N$ such that not only do we have $\left\|f\left(\mathcal{D}_{n}\right)-f(\mathcal{D})\right\| \leq$ $\varepsilon$ for all $n \geq N$, but that this estimate also holds for all $g$ in a small neighborhood of $f$. This together with the compactness of $K$ and the continuity of the maps $t \mapsto \mathrm{e}^{-t x^{2}}$ and $t \mapsto x \mathrm{e}^{-t x^{2}}$ implies the claim.

Remark 5.13. The arguments in the last parts of the proof can be used to show that we could also have equipped $\mathcal{S G O}_{n}$ with the topology that controls all derivatives of a super-semigroup map $\Phi$ and still would have obtained the same topological space. We find this interesting, because this is the topology that one usually considers on spaces of smooth maps.

5.2. Super-semigroups of operators and Euclidean field theories. In the context of supersymmetric Euclidean field theories of dimension (1|1) slight variations of the spaces $\mathcal{S G O}_{n}$ appear. We conclude this chapter by describing these spaces and showing that they have the same homotopy type as the spaces $\mathcal{S G O}_{n}$. More information about the axiomatic definition of supersymmetric EFTs will be given in the next chapter.

Definition 5.14. Let $\mathrm{TC}\left(\mathcal{H}_{n}\right)$ be the Banach algebra of trace class operators on $\mathcal{H}_{n}$, equipped with the trace or nuclear norm. Define $\mathcal{S G O}_{n}^{\text {TC }}$ to be the space of super-semigroup homomorphisms $\mathbb{R}_{>0}^{1 \mid 1} \rightarrow \mathrm{TC}\left(\mathcal{H}_{n}\right)$ with values in self-adjoint $C_{n^{-}}$ linear operators. As before, we consider the topology given by uniform convergence on compact subsets $K \subset \mathbb{R}_{>0}$. In the same way, we define $\mathcal{S} \mathcal{G O} \mathcal{O}_{n}^{\mathrm{HS}}$, where $\operatorname{TC}\left(\mathcal{H}_{n}\right)$ is replaced by $\operatorname{HS}\left(\mathcal{H}_{n}\right)$, the Banach algebra of Hilbert-Schmidt operators on $\mathcal{H}_{n}$, equipped with the Hilbert-Schmidt norm.

Examples 5.15. An SGO defined by an infinitesimal generator $\mathcal{D}$ lies in $\mathcal{S G O}{ }^{\mathrm{TC}}$ respectively $\mathcal{S G O}{ }^{\mathrm{HS}}$ if the eigenvalues of $\mathcal{D}$ converge to infinity sufficiently fast. This is, for example, the case for Dirac operators on closed spin manifolds, cf. $[17$, Chapter 3, §5].

Proposition 5.16. The injections

$$
\mathcal{S G O}_{n}^{\mathrm{TC}} \hookrightarrow \mathcal{S G O}_{n} \quad \text { and } \quad \mathcal{S G O}_{n}^{\mathrm{HS}} \hookrightarrow \mathcal{S G O}_{n}
$$

are homotopy equivalences. 
PROOF. We give the proof for $\mathcal{S} \mathcal{G O} \mathcal{O}_{n}^{\mathrm{TC}}$, the argument for $\mathcal{S} \mathcal{G} \mathcal{O}_{n}^{\mathrm{HS}}$ being very similar. The basic observation we use is that on operators of rank at most $k$ the operator norm and the nuclear norm are equivalent. More precisely,

$$
\|T\| \leq\|T\|_{\text {nuc }} \leq k \cdot\|T\|
$$

if $\operatorname{rank}(T) \leq k$. Namely, if $T=\sum_{i=1}^{k} s_{i}\left\langle\cdot, e_{i}\right\rangle f_{i}$ with $s_{1} \geq \cdots \geq s_{k} \geq 0$ for orthonormal systems $e_{i}$ and $f_{i}$, then $\|T\|=s_{1}$ and $\|T\|_{\text {nuc }}=\sum_{i=1}^{k} s_{i} \leq k s_{1}$. Denote by $\mathcal{S}^{(k)} \subset \mathcal{S} \mathcal{S O} \mathcal{O}_{n}^{\mathrm{TC}}$ the subspace of SGOs whose infinitesimal generator has domain of dimension $\leq 2 k$. By our basic observation, the topology on $\mathcal{S}^{(k)}$ is the same as the one we get by considering it as a subspace of $\mathcal{S G \mathcal { O } _ { n }}$. Using the identification $I: \mathcal{S G O} \mathcal{O}_{n} \approx \operatorname{Conf}_{n}$ and Remark 4.7 we see that $\mathcal{S}:=\operatorname{colim}_{k \rightarrow \infty} \mathcal{S}^{(k)} \simeq \mathcal{S} \mathcal{G} \mathcal{O}_{n}$. Hence, if we can show that $i: \mathcal{S} \hookrightarrow \mathcal{S G O}_{n}^{\mathrm{TC}}$ is a homotopy equivalence, then the same is true for $\mathcal{S G O}_{n}^{\mathrm{TC}} \hookrightarrow \mathcal{S G O}_{n}$. Define homotopies $\widetilde{H}$ and $H^{\mathrm{TC}}$ by the commutative diagram

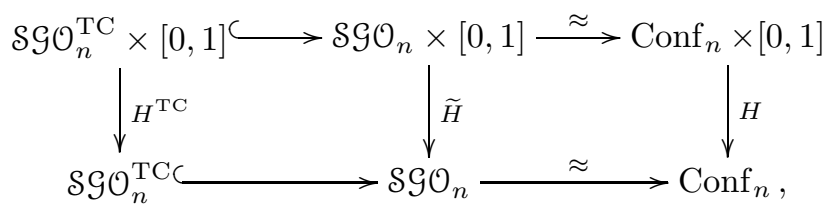

where $H$ is the homotopy used in the proof of Proposition 4.6. It is easy to see that the map $i^{-1} \circ H_{1}^{\mathrm{TC}}: \mathcal{S} \mathcal{S O}_{n}^{\mathrm{TC}} \rightarrow S$ is continuous. We claim that it is a homotopy inverse for $i$. The argument works exactly as in Remark 4.7. The only thing to check is that the homotopy $H^{\mathrm{TC}}$ is continuous (note that now we are using the topology that comes from the nuclear norm). For $t \neq 0$, the continuity follows from the continuity of $\widetilde{H}$ and the fact that $H^{\mathrm{TC}}$ locally maps to $\mathcal{S}^{(k)}$, for some $k$, as long as $t \neq 0$.

Let us now consider the case $t=0$. Assume $\Phi_{m} \rightarrow \Phi$ and $t_{m} \rightarrow 0$. For $C \subset \mathbb{R}$, we denote by $\Phi^{C}$ the SGO defined by the infinitesimal generator obtained from $I(\Phi)$ by omitting all labels in $\mathbb{R} \backslash C$. Let $K \subset \mathbb{R}_{>0}$ compact, $\varepsilon>0$. Write $\|\Phi\|:=\sup _{s \in K}\left(\|A(s)\|_{\text {nuc }}+\|B(s)\|_{\text {nuc }}\right)$. Since $\Phi \in \mathcal{S G \mathcal { O } ^ { \mathrm { TC } }}$, we can choose a big symmetric interval $C=[-\kappa, \kappa] \subset \mathbb{R}$ with $I(\Phi)_{\kappa}=0$ such that $\left\|\Phi-\Phi^{C}\right\|<\varepsilon$. Note that $\Phi_{m} \rightarrow \Phi$ also implies $\Phi_{m}^{C} \rightarrow \Phi^{C}$. The continuity of $\widetilde{H}$ implies that the restriction of $H^{\mathrm{TC}}$ to $\mathcal{S}^{(k)} \times[0,1]$ is continuous for all $k$. In particular, we have $H^{\mathrm{TC}}\left(\Phi_{m}^{C}, t_{m}\right) \rightarrow \Phi^{C}$. Now, choose $N \in \mathbb{N}$ such that for all $m>N$

$$
\left\|\Phi-\Phi_{m}\right\|<\varepsilon, \quad\left\|\Phi^{C}-\Phi_{m}^{C}\right\|<\varepsilon, \quad \text { and }\left\|H^{\mathrm{TC}}\left(\Phi_{m}^{C}, t_{m}\right)-\Phi^{C}\right\|<\varepsilon .
$$

It then follows that for all $m>N$

$$
\begin{aligned}
\left\|\Phi-H^{\mathrm{TC}}\left(\Phi_{m}, t_{m}\right)\right\| & \leq\left\|\Phi-\Phi^{C}\right\|+\left\|\Phi^{C}-H^{\mathrm{TC}}\left(\Phi_{m}^{C}, t_{m}\right)\right\|+\left\|H^{\mathrm{TC}}\left(\Phi_{m}^{\mathbb{R} \backslash C}, t_{m}\right)\right\| \\
& \leq 2 \varepsilon+\left\|\Phi_{m}-\Phi\right\|+\left\|\Phi-\Phi^{C}\right\|+\left\|\Phi^{C}-\Phi_{m}^{C}\right\| \\
& \leq 5 \varepsilon
\end{aligned}
$$

where we used that $\left\|H^{\mathrm{TC}}\left(\Phi_{m}^{\mathbb{R} \backslash C}, t_{m}\right)\right\| \leq\left\|\Phi_{m}^{\mathbb{R} \backslash C}\right\|=\left\|\Phi_{m}-\Phi_{m}^{C}\right\|$. This inequality follows directly from the definition of $H^{\mathrm{TC}}$ (provided we choose $\kappa \geq \min _{s \in K} 1 / \sqrt{2 s}$ ), which we can certainly do). Hence, $H^{\mathrm{TC}}\left(\Phi_{m}, t_{m}\right) \rightarrow \Phi$ in $\mathcal{S G O}_{n}^{\mathrm{TC}}$ so that $H^{\mathrm{TC}}$ is continuous.

Remark 5.17. In [28] the notation $1 \mid 1-\mathrm{EFT}^{-n}$ was used for the space $\mathcal{S G \mathcal { O } _ { n } ^ { \mathrm { HS } }}$. In this paper we want to reserve the notation 1|1-EFT for the "correct" notion of 
Euclidean field theories: we give a geometric definition of supersymmetric positive Euclidean field theories of dimension $(1 \mid 1)$ in the next section. It turns out that the resulting classifying spaces are homotopy equivalent to $\mathcal{S G O}_{n}^{\mathrm{TC}}$.

\section{Supersymmetric Euclidean field theories}

In this section we outline how the spaces $\mathcal{S G O}_{n}^{\mathrm{TC}}$ of super-semigroups of selfadjoint trace-class operators, studied in the previous section, are related to supersymmetric (1|1)-dimensional positive Euclidean field theories. Here we shall only treat the case $n=0$, so there is no Clifford algebra $C_{n}$ in the game. If $n \neq 0$, we would have to explain twisted Euclidean field theories that are defined in terms of certain 2-functors between 2-categories. This would lead too far afield in this paper and in the end, it would only give a different way in which the Clifford algebras $C_{n}$ arise.

The Atiyah-Segal definition of a d-dimensional topological field theory (TFT) is in terms of a symmetric monoidal functor $E: d$-Bord $\rightarrow$ Vect, or shorter,

$$
E \in \operatorname{Fun}^{\otimes}(d \text {-Bord, Vect })
$$

The target of the functor $E$ is the category Vect of vector spaces with tensor product. The domain category $d$-Bord is the bordism category whose objects are closed $(d-1)$-manifolds and whose morphisms are homeomorphism classes of compact $d$-dimensional bordisms with boundary decomposed into an incoming and an outgoing part. Composition is given by gluing bordism along the specified parts of the boundary. The symmetric monoidal structures is given by disjoint union of manifolds.

Our notion of a Euclidean field theory is based on such functors but we need to vary the source and target categories, mainly putting in geometry in the source, topology in the target and working in families. The new target category is easiest to describe and so we do this in the next subsection, where we shall recall some basic facts about Fréchet spaces and their (projective) tensor product.

In the following subsections we shall add geometry, families and supersymmetry into the definition of various bordism categories. In the last subsection we shall put all this information together and discuss the spaces 1|1-PEFT mentioned in our Main Theorem.

6.1. The symmetric monoidal category of Fréchet spaces. We start with a review of some basic facts on Fréchet spaces, see for example [15] or [20]. These are the complete topological vector spaces whose topology can be defined by an increasing sequence of semi-norms

$$
\rho_{1} \leq \rho_{2} \leq \cdots .
$$

Typical examples are Banach spaces (defined by a single norm) or spaces of continuous functions $C^{0}(X)$, for $X$ a union of an increasing sequence of compact sets $K_{n}$. In this case, the $\rho_{n}$ are given by the supremum norms on $K_{n}$. For a smooth manifold $M$, the Sobolev norms on $C^{\infty}(M)$ qualify for the $\rho_{s}$.

For a Banach space, the dual space again has a complete norm and hence a preferred topology. For a Fréchet space $V$ there are many interesting topologies on the continuous dual $V^{\prime}$. For example, one can define a strong topology on $V^{\prime}$ that agrees with the norm topology if $V$ is Banach. Moreover, a Fréchet space is Banach 
if and only if its strong dual is a Fréchet space and in general there is no topology on $V^{\prime}$ that always ends up with a Fréchet space!

The two basic theorems for Fréchet spaces are the closed graph theorem and the open mapping theorem: For a linear map $A: V \rightarrow W$ between Fréchet spaces the former says that $A$ is continuous if and only if its graph is closed and the latter says that $A$ is open if it is continuous and surjective. It follows that there is at most one Fréchet topology, finer than the subspace topology, on a subspace of a linear Hausdorff space and that a continuous bijective linear map between Fréchet spaces is an isomorphism.

There are many topologies on the algebraic tensor product of two topological vector spaces $V$ and $W$. We will only study the projective topology which is the finest topology on the algebraic tensor product $V \otimes_{\text {alg }} W$ for which the canonical bilinear map

$$
V \times W \rightarrow V \otimes_{\text {alg }} W
$$

is continuous. This means that a linear map $V \otimes_{\text {alg }} W \rightarrow G$ is continuous if and only if the corresponding bilinear map $V \times W \rightarrow G$ is continuous.

Lemma 6.1. If $V, W$ are Fréchet spaces then the completion $V \otimes W$ of the algebraic tensor product in the projective topology is again a Fréchet space, $[16$, 1781]. This tensor product is associative and commutative in the sense that SFr, the category of Fréchet spaces, becomes a symmetric monoidal category under this projective tensor product.

Examples 6.2. If $V$ is Fréchet then $C^{\infty}(M ; V) \cong C^{\infty}(M) \otimes V$. In particular,

$$
C^{\infty}(M \times N) \cong C^{\infty}(M) \otimes C^{\infty}(N)
$$

for any smooth manifolds $M$ and $N$. In fact, in the graded category these statements continue to hold for super manifolds.

If $V, W$ happen to be Hilbert spaces then $\bar{V} \otimes W$ can be identified with the space of trace-class operators from $V$ to $W$ by the canonical map. Note that the Hilbert tensor product of $\bar{V}$ and $W$ leads to the space of Hilbert-Schmidt operators. If $V=$ $W$ is infinite-dimensional, the inner product extends to a continuous homomorphism on the former but not the latter space, which is why we cannot use the monoidal category of Hilbert spaces as the target for our field theories.

The following lemma will be used in the Main Theorem of this section in order to pick out one Fréchet space from a zoo of possibilities.

Lemma 6.3. Let $V$ be a Fréchet space and $h: \bar{V} \otimes V \rightarrow \mathbb{C}$ a continuous linear map that is Hermitian, positive definite and induces an (algebraic) isomorphism $\bar{V} \rightarrow V^{\prime}$. Then $(V, h)$ is a Hilbert space whose norm topology agrees with the original Fréchet topology.

Before we give the proof of this lemma, we point out the case $V=C^{\infty}(M)$ for a compact Riemannian manifold $M$. The inclusion $C^{\infty}(M) \hookrightarrow L^{2}(M)$ is continuous but not open. It induces a positive definite inner product $h$ on $V$ which satisfies all the conditions except that $\bar{V} \rightarrow V^{\prime}$ is only $1-1$ and not onto ( $V^{\prime}$ is a space of distributions). So the following proof of Lemma 6.3 has to decisively use that $\bar{V} \rightarrow V^{\prime}$ is an isomorphism.

Proof. It is clear that the composition

$$
V \stackrel{\Delta}{\rightarrow} V \times V \stackrel{h}{\rightarrow} \mathbb{C}
$$


is continuous and therefore the identity is a continuous map $V \rightarrow H$, where $V$ denotes the original Fréchet space and $H$ denotes $V$ with the norm topology given by $\|v\|^{2}:=h(v, v)$. We will see in the next paragraph that the pre-Hilbert space $H$ is actually complete. Hence we can apply the open mapping theorem to the identity map $V \rightarrow H$ and conclude that it is an isomorphism, as asserted.

Since the identity map $V \rightarrow H$ is continuous, we have an inclusion $\iota: H^{\prime} \hookrightarrow V^{\prime}$. The composition $\bar{H} \hookrightarrow V^{\prime}$ of $\iota$ with the canonical map $c: \bar{H} \rightarrow H^{\prime}$ is surjective since it is equal to the composition $\bar{H}=\bar{V} \rightarrow V^{\prime}$ which is surjective by assumption. It follows that the canonical map $\bar{H} \rightarrow H^{\prime}$ is surjective and hence the pre-Hilbert space $H$ is complete.

6.2. Riemannian bordism categories. In [29] we defined the Riemannian bordism category $d$-RB as an internal category over the 2-category of symmetric monoidal categories. This means that we have

(0) objects: closed piecewise smooth $(d-1)$-manifolds $Y^{\mathrm{c}}$ with a $d$-dimensional Riemannian bicollar $Y$.

$\left(1_{\mathrm{v}}\right)$ vertical 1-morphisms: germs of isometries between objects $\left(Y, Y^{\mathrm{c}}\right)$.

$\left(1_{\mathrm{h}}\right)$ horizontal 1-morphisms: $d$-dimensional Riemannian bordisms $\Sigma$ between such objects.

(2) 2-morphisms: germs of isometries of horizontal 1-morphisms $\Sigma$.

This 2-categorical language is very important if one wants to make twisted field theories precise, for example for degree $n \neq 0$. In this paper we decided to skip this complication and hence we collapse the above structure to that of a symmetric monoidal category. We hope that using the same notation $d$-RB as in [29] will not be confusing for the readers. Here are the detailed definitions used in this paper. One important aspect is that all our manifolds are without boundary, making it easier to deal with geometric structures.

Definition 6.4. The objects of the category $d$-RB are quadruples $\left(Y, Y^{\mathrm{c}}, Y^{ \pm}\right)$, where $Y$ is a Riemannian $d$-manifold (usually noncompact) and $Y^{\mathrm{c}} \subset Y$ is a compact codimension 1 submanifold which we call the core of $Y$. Moreover, we require that $Y \backslash Y^{\mathrm{c}}=Y^{+} \amalg Y^{-}$, where $Y^{ \pm} \subset Y$ are disjoint open subsets whose closures contain $Y^{\mathrm{c}}$. An isomorphism in $d$-RB from $\left(Y_{0}, Y_{0}^{\mathrm{c}}, Y_{0}^{ \pm}\right)$to $\left(Y_{1}, Y_{1}^{\mathrm{c}}, Y_{1}^{ \pm}\right)$is the germ of an invertible isometry $f: W_{0} \rightarrow W_{1}$. Here $W_{j} \subset Y_{j}$ are open neighborhoods of $Y_{j}^{\mathrm{c}}$ and $f$ is required to send $Y_{0}^{\mathrm{c}}$ to $Y_{1}^{\mathrm{c}}$ and $W_{0}^{ \pm}$to $W_{1}^{ \pm}$where $W_{j}^{ \pm}:=W_{j} \cap Y_{j}^{ \pm}$. As usual for germs, two such isometries represent the same isomorphism if they agree on some smaller open neighborhood of $Y_{0}^{\mathrm{c}}$ in $Y_{0}$.

Disjoint union makes this a symmetric monoidal groupoid, the invertible part of $d$-RB. We shall now introduce the general morphisms of $d$-RB as (isometry classes of) certain bordisms and we shall rediscover the above isomorphisms as very special types of bordisms.

Definition 6.5. A Riemannian bordism from $Y_{0}=\left(Y_{0}, Y_{0}^{\mathrm{c}}, Y_{0}^{ \pm}\right)$to $Y_{1}=$ $\left(Y_{1}, Y_{1}^{\mathrm{c}}, Y_{1}^{ \pm}\right)$is a triple $\left(\Sigma, i_{0}, i_{1}\right)$ consisting of a Riemannian $d$-manifold $\Sigma$ and smooth maps $i_{j}: W_{j} \rightarrow \Sigma$. Here $W_{j} \subset Y_{j}$ are open neighborhoods of the cores $Y_{j}^{\text {c }}$. Letting $i_{j}^{ \pm}: W_{j}^{ \pm} \rightarrow \Sigma$ be the restrictions of $i_{j}$ to $W_{j}^{ \pm}:=W_{j} \cap Y_{j}^{ \pm}$, we require that (+) $\quad i_{j}^{+}$are isometric embeddings into $\Sigma \backslash i_{1}\left(W_{1}^{-} \cup W_{1}^{\mathrm{c}}\right)$ and

(c) the core $\Sigma^{\mathrm{c}}:=\Sigma \backslash\left(i_{0}\left(W_{0}^{+}\right) \cup i_{1}\left(W_{1}^{-}\right)\right)$is compact. 


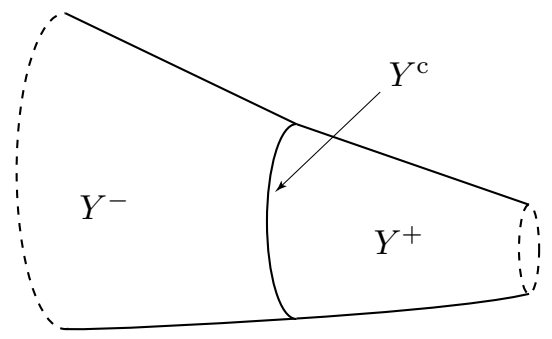

Figure 1. An object $\left(Y, Y^{\mathrm{c}}, Y^{ \pm}\right)$of 2-RB

Particular bordisms are given by isometries $f: W_{0} \rightarrow W_{1}$ as above, namely by using $\Sigma=W_{1}, i_{1}=\operatorname{id}_{W_{1}}$ and $i_{0}=f$. Note that in this case the images of $i_{0}^{+}$and $i_{1}^{+}$are not disjoint but we didn't require this condition.

Below is a picture of a Riemannian bordism; we usually draw the domain of the bordism to the right of its range, since we want to read compositions of bordisms, like compositions of maps, from right to left. Roughly speaking, a Riemannian bordism between objects $Y_{0}$ and $Y_{1}$ of $d$-RB is just an ordinary bordism $\Sigma^{\mathrm{c}}$ from $Y_{0}^{\mathrm{c}}$ to $Y_{1}^{\mathrm{c}}$ equipped with a Riemannian metric, thickened up a little bit near its boundary to make gluing possible.

The composition of two Riemannian bordisms $\Sigma: Y_{0} \rightarrow Y_{1}$ and $\Sigma^{\prime}: Y_{1} \rightarrow Y_{2}$ is defined as follows. Consider the maps $i_{1}: W_{1} \rightarrow \Sigma$ and $i_{1}^{\prime}: W_{1}^{\prime} \rightarrow \Sigma^{\prime}$ that are part of the data for our Riemannian bordisms. Here $W_{1}, W_{1}^{\prime} \subset Y_{1}$ are open neighborhoods of $Y_{1}^{\mathrm{c}}$ and we set $W_{1}^{\prime \prime}:=W_{1} \cap W_{1}^{\prime}$. Our conditions guarantee that $i_{1}$ and $i_{1}^{\prime}$ restrict to isometric embeddings of $\left(W_{1}^{\prime \prime}\right)^{+}:=W_{1}^{\prime \prime} \cap Y_{1}^{+}$. We use these isometries to glue $\Sigma$ and $\Sigma^{\prime}$ along $W_{1}^{\prime \prime}$ to obtain $\Sigma^{\prime \prime}$ defined as follows:

$$
\Sigma^{\prime \prime}:=\left(\Sigma^{\prime} \backslash i_{1}^{\prime}\left(\left(W_{1}^{\prime}\right)^{+} \backslash\left(W_{1}^{\prime \prime}\right)^{+}\right)\right) \cup_{\left(W_{1}^{\prime \prime}\right)}+\left(\Sigma \backslash i_{1}\left(W_{1}^{-} \cup W_{1}^{\mathrm{c}}\right)\right)
$$

The maps $i_{0}: W_{0} \rightarrow \Sigma$ and $i_{2}: W_{2} \rightarrow \Sigma^{\prime}$ can be restricted to maps (on smaller open neighborhoods) into $\Sigma^{\prime \prime}$ and they induce isometric embeddings as required

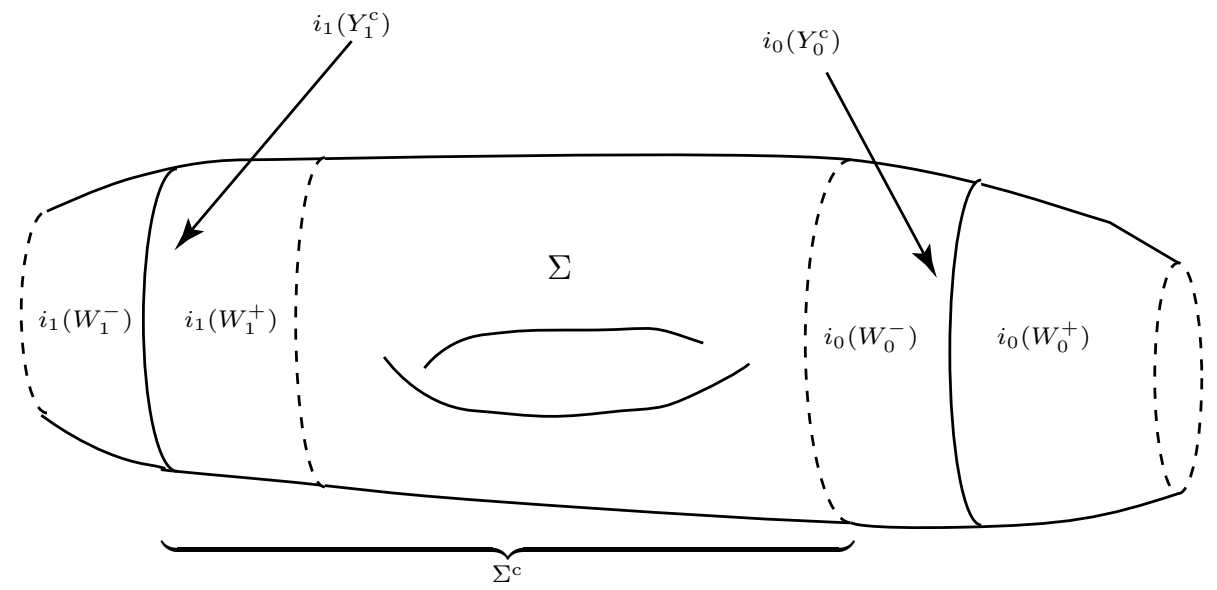

Figure 2. A 2-dimensional Riemannian bordism $\left(\Sigma, i_{0}, i_{1}\right)$ 
by our conditions. This makes $\Sigma^{\prime \prime}$ a Riemannian bordism from $Y_{0}$ to $Y_{2}$ using the following remark.

Remark 6.6. We point out that the conditions $(+)$ and (c) in the above definition of a Riemannian bordism also make sure that the composed bordism is again a Hausdorff space. In other words, gluing two topological spaces along open subsets preserves conditions like "locally homeomorphic to $\mathbb{R}^{n}$ " and structures like Riemannian metrics. However, it can happen that the glued up space is not Hausdorff, for example if one glues two copies of $\mathbb{R}$ along the interval $(0,1)$. The reader is invited to check that our claim follows from the following easy lemma.

Lemma 6.7. Let $X, X^{\prime}$ be manifolds and let $U$ be an open subset of $X$ and $X^{\prime}$. Then $X \cup_{U} X^{\prime}$ is a manifold if and only if the natural map $U \rightarrow X \times X^{\prime}$ sends $U$ to a closed set.

An isometry between Riemannian bordisms $\Sigma, \Sigma^{\prime}: Y_{0} \rightarrow Y_{1}$ is a germ of a triple of isometries

$$
F: X \rightarrow X^{\prime} \quad f_{0}: V_{0} \rightarrow V_{0}^{\prime} \quad f_{1}: V_{1} \rightarrow V_{1}^{\prime} .
$$

Here $X \subset \Sigma$ (respectively $V_{j} \subset W_{j} \cap i_{j}^{-1}(X) \subset Y_{j}$ ) are open neighborhoods of $\Sigma^{\mathrm{c}}$ (respectively $Y_{j}^{\mathrm{c}}$ ) and similarly for $X,{ }^{\prime} V_{0},{ }^{\prime} V_{1}^{\prime}$. We require the conditions for $f_{j}$ to be an isomorphism in $d$-RB as in Definition 6.4 and that the following diagram commutes:

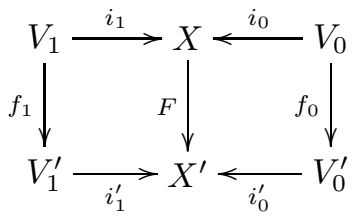

Two such triples $\left(F, f_{0}, f_{1}\right)$ and $\left(G, g_{0}, g_{1}\right)$ represent the same germ if there there are smaller open neighborhoods $X^{\prime \prime}$ of $\Sigma^{\mathrm{c}} \subset X$ and $V_{j}^{\prime \prime}$ of $Y_{j} \subset V_{j} \cap i_{j}^{-1}\left(X^{\prime \prime}\right)$ such that $F$ and $G$ agree on $X^{\prime \prime}$, and $f_{j}$ and $g_{j}$ agree on $V_{j}^{\prime \prime}$.

Isometries with a germ of the form $\left(F, \mathrm{id}_{V_{0}}, \mathrm{id}_{V_{1}}\right)$ are referred to as being rel boundary.

Definition 6.8. We define the morphisms in $d$-RB to be isometry classes (rel boundary) of Riemannian bordisms as defined above. The composition is welldefined and associative on such isometry classes. It is not hard to check that the isomorphisms in $d$-RB all come from isometries as explained above.

Disjoint union makes $d$-RB into a symmetric monoidal category.

Remark 6.9. It is extremely important to observe that there are no orientations used to distinguish between incoming and outgoing boundaries. From our perspective, it is an unfortunate historical coincidence that in many papers orientations are mixed up with categorical source and target information. In the above approach the convention is that incoming boundaries of $\Sigma$ have the --side on the inside whereas outgoing boundaries have the --side on the outside. In the figures for $d=1$ below, one can also remember this by noting that the $\bullet$, representing the core of a point, is on the outside of the pictured bordism if and only if it is an outgoing boundary component.

It will also become clear later that this is the correct convention when studying functors into infinite-dimensional vector spaces. 
The following object pt $\in 1$-RB is clearly the unique connected nonempty object up to isomorphism, implying that $\pi_{0}(1-\mathrm{RB})=\mathbb{N}_{0}$ :

$$
\mathrm{pt}:=\left(\mathrm{pt}, \mathrm{pt}^{\mathrm{c}}, \mathrm{pt}^{ \pm}\right):=\left(\mathbb{R},\{0\}, \mathbb{R}_{ \pm}\right)
$$

In fact, pt is the germ around $\{0\}$ and a good picture is given by drawing the core $\mathrm{pt}^{\mathrm{c}}$ as a $\bullet$, the + collar $\mathrm{pt}^{+}$as a dashed line and ignoring the - collar $\mathrm{pt}^{-}$altogether:

$$
\text { - } \stackrel{\mathrm{pt}}{-}
$$

This choice comes from the fact that only the +collar is assumed to be embedded when considering Riemannian bordisms in and out of pt.

We draw the interval of length $t \geq 0$, a morphism $I_{t} \in 1-\mathrm{RB}(\mathrm{pt}, \mathrm{pt})$ as follows:

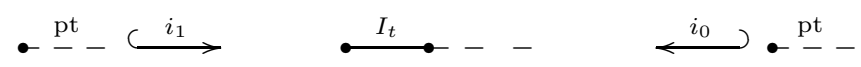

The underlying Riemannian manifold of $I_{t}$ is $\mathbb{R}$, the incoming embedding $i_{0}$ is given by translation by $t$ and the outgoing embedding $i_{1}$ is the identity. As for the object pt we only draw the +collars embedded in $I_{t}$ (and not the images of the -collars that aren't assumed to be embedded). The corresponding topological bordism $I_{t}^{\mathrm{c}}$ is the usual compact interval $[0, t]$, obtained by removing the dashed line on the right. Here we only needed to remove the image of the +collar under the embedding $i_{0}^{+}$ since the image of the - collar under $i_{1}$ isn't even drawn. Note that $I_{0}$ represents the identity morphism for the object pt.

Two pictures of important morphisms in 1-RB are as follows:

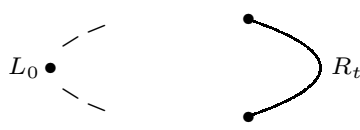

We draw such bordisms so that their source is on the right and their target is on the left, to make it easier to compare pictures with algebraic formulas. We call $R_{t}: \varnothing \rightarrow \mathrm{pt} \amalg \mathrm{pt}$ the right elbow and $L_{t}: \mathrm{pt} \amalg \mathrm{pt} \rightarrow \varnothing$ the left elbow. It is very important to notice that $R_{t}$ is only defined for $t>0$, for $t=0$ condition ( + ) in the definition of a Riemannian bordism is violated. However, it is satisfied for $L_{0}$ which is an example of a noninvertible Riemannian bordism for which the core is a single point (just like for isometries of pt).

Remark 6.10. Originally we thought that the intervals $I_{t}$ are all one needs to understand 1-manifolds: just pick a triangulation that decomposes any 1-manifold into intervals. However, one should be more careful and rather think of manifolds decomposed into handles, then 0- and 1-handles are relevant for 1-manifolds. Categorically, this can also be seen by observing that there is no way to recover $R_{t}$ just from intervals. To deal with this problem, we introduced the 'adjunction transformations' on the bordism categories and on Vect in our original approach [28]. We required the functor $E$ to preserve these extra structures, leading to a more awkward definition.

However, if one defines the category 1-RB as above, these additional properties are automatically satisfied as one can see by playing with the morphism $L_{0}$. We now prefer this approach because it introduces the concept of handle decomposition of 1-manifolds in a precise fashion: In the following Theorem, think of $L_{0}$ as associated to a 0-handle (of length 0 ) and $R_{t}$ as associated to 1-handles (of length $t>0$ ). The 
relations come from Morse cancelling a pair of such handles and the orientation reversing isometry of an interval.

Theorem 6.11. The symmetric monoidal category 1-RB is generated by the object $\mathrm{pt}$ and the morphisms $L_{0}$ and $R_{t}, t \in \mathbb{R}_{>0}$, subject to the following relations

- $L_{0}$ and $R_{t}$ are symmetric, i.e., they interact as follows with the symmetry braiding $\sigma$ of 1-RB: $L_{0}=L_{0} \circ \sigma$ and $R_{t}=\sigma \circ R_{t}$.

- $R_{t_{1}+t_{2}}$ equals the composition $R_{t_{1}} \circ L_{0} R_{t_{2}}:=\left(I_{0} \amalg L_{0} \amalg I_{0}\right) \circ\left(R_{t_{1}} \amalg R_{t_{2}}\right)$ :

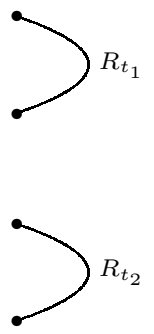

PROOF. Having a set of generators for a symmetric monoidal category B means that the symmetric monoidal category freely generated by this set comes with an essentially surjective functor to B. In our case, this is true since

(1) any object in 1-RB is isometric to the disjoint union of points,

(2) any morphism in 1-RB is isometric to the disjoint union of Riemannian circles and intervals,

(3) the moduli space of intervals (and circles) is $(0, \infty)$ given by the length $t$,

(4) all ways of assigning source and target to an interval are given by $I_{t}, L_{t}, R_{t}$. Moreover, $L_{0}$ and $R_{t}$ can be composed to give $I_{t}, L_{t}$,

(5) circles are obtained from composing $L_{t}$ and $R_{t}$.

To find the relations between the generators $L_{0}$ and $R_{t}$ we have to understand their isometries relative boundary: These are clearly given by reflections and the first set of relations arises. The second relation is a geometric version of Morse cancellation if one thinks of $L_{0}$ as associated to a 0-handle (of length 0 ) and $R_{t}$ as associated to 1-handles (of length $t>0$ ). In this dimension, this is the only possible Morse cancellation. With some care, the usual statement of handlebody theory, namely that any two handle decompositions of a manifold differ by a sequence of Morse cancellations, leads to a proof of our theorem.

For those readers who are not familiar with the notion of generators and relations for symmetric monoidal categories, the following consequence of Theorem 6.11 can serve as a definition.

Corollary 6.12. For any symmetric monoidal category $\mathrm{C}$, the groupoid of symmetric monoidal functors from 1-RB to $\mathrm{C}$ (and natural isomorphisms) is equivalent to a certain groupoid $\mathrm{C}^{3}$ :

$$
\operatorname{Fun}^{\otimes}(1-\mathrm{RB}, \mathrm{C}) \simeq \mathrm{C}^{3}
$$

Here the objects of $\mathrm{C}^{3}$ are triples $\left(V, \mu, \rho_{t}\right)$ where $V \in \mathrm{C}$ and

$$
\mu: V \otimes V \rightarrow \mathbb{1} \text { respectively } \rho_{t}: \mathbb{1} \rightarrow V \otimes V, t \in \mathbb{R}_{>0}
$$

are symmetric morphisms in $\mathrm{C}$ subject to the relations

$$
\rho_{t_{1}+t_{2}}=\rho_{t_{1}} \circ_{\mu} \rho_{t_{2}}:=(\mathbb{1} \otimes \mu \otimes \mathbb{1}) \circ\left(\rho_{t_{1}} \otimes \rho_{t_{2}}\right)
$$


shown in (5). Note that this figure explains how to use a fixed morphism $\mu$ to introduce a monoidal structure on $\mathrm{C}(\mathbb{1}, V \otimes V)$, denoted by $\circ_{\mu}$ above. This works in any symmetric monoidal category $\mathrm{C}$ and our relation says that $\rho$ is a homomorphism of monoids $\mathbb{R}_{>0} \rightarrow \mathrm{C}(\mathbb{1}, V \otimes V)$.

The morphisms $\mathrm{C}^{3}\left(\left(V, \mu, \rho_{t}\right),\left(V,^{\prime} \mu,^{\prime} \rho_{t}^{\prime}\right)\right)$ are isomorphisms in $\mathrm{C}\left(V, V^{\prime}\right)$ for which the obvious diagrams involving $\mu, \mu, \rho_{t}^{\prime}, \rho_{t}^{\prime}$ commute.

In particular, applying this result to $C=$ Vect, we see that 1-dimensional Riemannian field theories form a groupoid equivalent to Vect ${ }^{3}$. For example, given a Hilbert space $V$ with inner product $\mu$ and an operator $A$ on $V$, one can form the semigroup of operators $\hat{\rho}_{t}:=\exp (-t A)$ and hope that it comes from $\rho_{t} \in V \otimes V$. There are a few problems with this example.

(1) Since $\mu$ is a bilinear pairing, rather then Hermitian, it qualifies as an inner product only over the real numbers. To relate to Hermitian pairings over the complex numbers, one needs to introduce orientations on the Riemannian manifolds which we do in [12].

(2) If we are using the algebraic tensor product on Vect then $V \otimes V$ can be identified with the finite rank operators, putting severe restrictions on $A$. This problem is resolved by working in the category of Fréchet space with projective tensor product as explained in Section 6.1. Note that the monoidal category of Hilbert spaces only works in finite dimensions because in infinite dimensions the pairing $\mu$ is not defined on the Hilbert tensor product of $V$ with itself: this tensor product is isomorphic to the Hilbert-Schmidt operators on $V$ whereas the projective tensor product gives trace-class operators on $V$. In the translation, the pairing $\mu$ turns into the trace which is defined on this projective tensor product but doesn't make sense for all Hilbert-Schmidt operators.

(3) Most importantly, if we want that all Riemannian field theories arise in this manner, we have to make sure that the elements $\rho_{t}$ vary continuously, or even smoothly, in $t$. Then the semigroup $\hat{\rho}_{t}$ can be differentiated to give an infinitesimal generator $A$. This requirement leads naturally to include family versions of the categories $d-\mathrm{RB}$, introduced in the next two subsections.

6.3. Families of rigid geometries. Let $M$ denote one of the categories of manifolds Man, super manifolds SMan, or complex super manifolds csM. The latter are defined in [6] in terms of an ordinary $p$-manifold, together with a sheaf of commutative superalgebras over $\mathbb{C}$ that are locally isomorphic to

$$
C^{\infty}\left(\mathbb{R}^{p \mid q}\right) \otimes \mathbb{C}
$$

In this case, $p \mid q$ is again referred to as the superdimension. One should think of these manifolds as having $p$ real coordinates and $q$ complex coordinates and they all arise from complex vector bundles over ordinary manifolds.

Here and in the following, a manifold will stand for an object in $\mathrm{M}$ (which has no boundary). Let $G$ be a group object in M, i.e., a Lie group, Lie supergroup or Lie cs-group. We want to think of a $G$-action on a fixed manifold $\mathbb{M}$ as the local model for rigid geometries with isometry group $G$. This idea is very well explained in [30] and goes back to Klein's Erlangen program.

Definition 6.13. A $(G, \mathbb{M})$-structure on a manifold $Y$ is a maximal atlas consisting of charts which are diffeomorphisms

$$
Y \supseteq U_{i} \stackrel{\varphi_{i}}{\cong} V_{i} \subseteq \mathbb{M}
$$


between open subsets of $Y$ and open subsets of $\mathbb{M}$ such that the $U_{i}$ 's cover $Y$ and for all $i, j$ the transition function

$$
\mathbb{M} \supseteq \varphi_{i}\left(U_{i} \cap U_{j}\right) \stackrel{\varphi_{j} \circ \varphi_{i}^{-1}}{\longrightarrow} \varphi_{j}\left(U_{i} \cap U_{j}\right) \subseteq \mathbb{M}
$$

is given by an element $g \in G$, it is the restriction of the map

$$
\mathbb{M}=\mathrm{pt} \times \mathbb{M} \stackrel{g \times \mathrm{id}}{\longrightarrow} G \times \mathbb{M} \rightarrow \mathbb{M}
$$

for some $\mathrm{g}: \mathrm{pt} \rightarrow G$. Here $G \times \mathbb{M} \rightarrow \mathbb{M}$ is the action map and this careful formulation is particularly relevant for super manifolds.

In the category of super manifolds (or cs-manifolds), any morphism pt $\rightarrow G$ factors uniquely through $G_{\text {red }} \subset G$. As a consequence, a $(G, \mathbb{M})$-structure on a super manifold $Y$ is the same thing as a $\left(G_{\text {red }}, \mathbb{M}\right)$-structure on $Y$. Therefore, the relevant notion for super manifolds is that of families of $(G, \mathbb{M})$-manifolds which we define next.

Definition 6.14. A family of $(G, \mathbb{M})$-manifolds is a morphism $p \in \mathrm{M}(Y, S)$ together with a maximal atlas consisting of charts which are diffeomorphisms $\varphi_{i}$ between open subsets of $Y$ and open subsets of $S \times \mathbb{M}$ making the following diagram commutative:

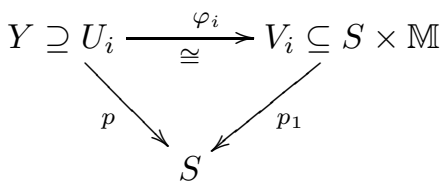

We require that the open sets $U_{i}$ cover $Y$ and that for all $i, j$ the transition function

$$
S \times \mathbb{M} \supseteq \varphi_{i}\left(U_{i} \cap U_{j}\right) \stackrel{\varphi_{j} \circ \varphi_{i}^{-1}}{\longrightarrow} \varphi_{j}\left(U_{i} \cap U_{j}\right) \subseteq S \times \mathbb{M}
$$

is of the form $(s, m) \mapsto\left(s, g_{i j}(s) m\right)$, where $g_{i j} \in \mathrm{M}\left(p\left(U_{i} \cap U_{j}\right), G\right)$. We note that the conditions imply in particular that $p$ is a submersion and $p\left(U_{i} \cap U_{j}\right) \subseteq S$ are open.

If $Y \rightarrow S$ and $Y^{\prime} \rightarrow S^{\prime}$ are two families of $(G, \mathbb{M})$-manifolds, an isometry between them is a pair of maps $(f, \hat{f})$ making the following diagram commutative:

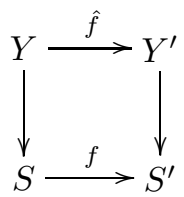

We require that $\hat{f}$ preserves the fiberwise $(G, \mathbb{M})$-structure in the following sense: There are charts $\left(U_{i}, \varphi_{i}\right)$, respectively $\left(U_{i}^{\prime}, \varphi_{i}^{\prime}\right)$, of $Y$, respectively $Y^{\prime}$, such that $\hat{f}\left(U_{i}\right) \subseteq U_{i}^{\prime}$ and $\varphi_{i}^{\prime} \circ \hat{f} \mid U_{i} \circ \varphi_{i}^{-1}$ is of the form $(s, m) \mapsto\left(f(s), g_{i}(s) m\right)$ for some $g_{i} \in \mathrm{M}\left(p\left(U_{i}\right), G\right)$.

The result is a category $\mathrm{M}(G, \mathbb{M})$ of families of $(G, \mathbb{M})$-manifolds and their isometries. It is easy to see that the forgetful functor $\mathrm{M}(G, \mathbb{M}) \rightarrow \mathrm{M}$ is a Grothendieck fibration, i.e., pullbacks exist.

Example 6.15. In the category Man of manifolds, we can consider $\mathbb{M}:=\mathbb{E}^{d}$, the $d$-dimensional Euclidean space. Let $G:=\operatorname{Iso}\left(\mathbb{E}^{d}\right)$ be the isometry group of $\mathbb{E}^{d}$, which is the Euclidean group of translations, reflections and rotations of $\mathbb{E}^{d}$. A 
Euclidean structure on a smooth $d$-manifold $Y$ is an $\left(\operatorname{Iso}\left(\mathbb{E}^{d}\right), \mathbb{E}^{d}\right)$-structure in the above sense. It is clear that such an atlas determines a flat Riemannian metric on $Y$ by transporting the standard metric on $\mathbb{E}^{d}$ to $U_{i}$ via the diffeomorphism $\varphi_{i}$. Conversely, a flat Riemannian metric can be used to construct such an atlas.

We want to give a super version of this geometric structure, at least in dimension $1 \mid 1$. We would like the local model to be $\mathbb{M}=\mathbb{R}^{1 \mid 1}$ and the translational part of the isometry group to be $\mathbb{R}^{1 \mid 1}$ with the group structure from Definition 5.3. This is the right group structure for the current setting because its Lie algebra is free on one odd generator $D$ with square $\partial_{t}$, the infinitesimal generator of the translation group $\mathbb{R}$.

In Iso $\left(\mathbb{E}^{1}\right)$ we have the reflection that takes $t \mapsto-t$, physically it's the time reversal. If we want an automorphism of $\mathbb{R}^{1 \mid 1}$ that induces this reflection on the reduced group $\mathbb{R}$ then it has to induce an action on the Lie algebra that sends $D \mapsto \mathrm{i} D$, where $\mathrm{i}$ is a square root of -1 . Thus super-time $\mathbb{R}^{1 \mid 1}$ must be a cs-Lie group if we want to include a super-time reversal.

Example 6.16. In the category csM of cs-manifolds, consider the following rigid geometry. Define Euclidean cs-space of dimension 1|1 to be

$$
\mathbb{M}:=\mathbb{E}^{1 \mid 1}:=\mathbb{R}_{\mathrm{cs}}^{1 \mid 1}
$$

and let $G:=\mathbb{R}_{\mathrm{cs}}^{1 \mid 1} \rtimes \mathbb{Z} / 4$ be the isometry group. So by definition, Iso $\left(\mathbb{E}^{1 \mid 1}\right):=G$, where the group structure on the translational part $\mathbb{R}_{\mathrm{cS}}^{1 \mid 1}$ comes from Definition 5.3. The "rotational" part $\mathbb{Z} / 4$ is generated by the pin generator pg which is the automorphism of the group $\mathbb{R}_{\mathrm{cs}}^{1 \mid 1}$ given by

$$
\operatorname{pg}(t, \theta):=(-t,-\mathrm{i} \theta), \quad \mathrm{i}^{2}=-1 .
$$

One checks that $G$ acts on $\mathbb{M}$ by first rotating and then left translating. Then

$$
\operatorname{csM}\left(\operatorname{Iso}\left(\mathbb{E}^{1 \mid 1}\right), \mathbb{E}^{1 \mid 1}\right)
$$

is the category of families of Euclidean cs-manifolds.

Note that the underlying reduced Lie group of Iso $\left(\mathbb{E}^{1 \mid 1}\right)$ is $\mathbb{R} \rtimes \mathbb{Z} / 4$, a central double covering of Iso $\left(\mathbb{E}^{1}\right)$. Hence the underlying reduced manifold of a Euclidean 1|1-manifold comes equipped with a Riemannian metric and pin-structure (more precisely, a tangential $\mathrm{Pin}^{-}(1)$-structure).

Remark 6.17. We used to think of a 1|1-dimensional Euclidean structure in terms of an odd complex vector field $Q$ that can be locally written as $\partial_{\theta}+\theta \partial_{t}$ and that is well-defined up to multiplication by complex numbers of length 1 . It is actually not difficult to see that the above group $G:=\mathbb{R}_{\mathrm{cs}}^{1 \mid 1} \rtimes \mathbb{Z} / 4$ is exactly the group of isometries of this geometric structure on $\mathbb{R}_{\mathrm{cs}}^{1 \mid 1}$.

We recently decided to use the approach via rigid geometries because in this setting one can easily define $d \mid \delta$-dimensional Euclidean manifolds (and bordism categories), see [29].

For later use in the definition of real field theories, we shall introduce the concept of complex conjugation on categories $\mathrm{M}(G, \mathbb{M})$. Recall that for a cs-manifold $S$, the complex conjugate cs-manifold $\bar{S}$ has the same real sheaf of functions but the complex numbers act by precomposing with complex conjugation. If $S$ is a manifold or super manifold, we define $\bar{S}:=S$. 
Definition 6.18. A real structure on a manifold $S$ (in Man, SMan or csM) is a isomorphism

$$
r_{S}: S \rightarrow \bar{S} \text { such that } \bar{r} \circ r=\operatorname{id}_{S}
$$

For example, if $\mathcal{O}(S)$ is the real sheaf of functions on a super manifold $S$ then there is a canonical cs-manifold $S_{\mathrm{cs}}$ with $\left(S_{\mathrm{cs}}\right)_{\text {red }}=S_{\text {red }}$ and sheaf of functions $\mathcal{O}\left(S_{\text {cs }}\right)=\mathcal{O}(S) \otimes \mathbb{C}$. The usual real structure on $\mathbb{C}$ then induces a real structure on $S_{\mathrm{cs}}$ which is the identity on the reduced manifold.

A morphism $f: S^{\prime} \rightarrow S$ is real if $r_{S} \circ f=f \circ r_{S^{\prime}}$. The above functor $S \mapsto$ $S_{\mathrm{cs}}$ gives an equivalence of categories between SMan and the category of real csmanifolds (with real structures that are the identity on the reduced part) and real morphisms. In the following, we shall not require our real structures to be the identity on the reduced part.

A real structure on a local model $(G, \mathbb{M})$ for rigid geometry is a real structure on $G$ and a real structure on $\mathbb{M}$ such that all structure maps (group multiplication, unit, inverse, group action) are real.

Lemma 6.19. A real structure on $(G, \mathbb{M})$ induces an involution on the category $\mathrm{M}(G, \mathbb{M})$ that extends the complex conjugation functor $Y \mapsto \bar{Y}$ on total spaces of families of $(G, \mathbb{M})$-manifolds. In particular, the forgetful functor $\mathrm{M}(G, \mathbb{M}) \rightarrow \mathrm{M}$ respects these complex conjugations.

Proof. Let $\varphi_{i}: U_{i} \rightarrow V_{i}$ be charts for a family $p: Y \rightarrow S$ as in Definition 6.14 above with transition functions coming from $g_{i j} \in \mathrm{M}\left(p\left(U_{i} \cap U_{j}\right), G\right)$. Then

$$
\bar{Y} \supseteq \bar{U}_{i} \underset{\bar{\varphi}_{i}}{\cong} \bar{V}_{i} \stackrel{\mathrm{id}_{S} \times r_{\mathbb{M}}}{\cong} W_{i} \subseteq \bar{S} \times \mathbb{M}
$$

are charts for $\bar{Y} \rightarrow \bar{S}$. One can easily check that, as required for a family of $(G, \mathbb{M})$ manifolds, the transition functions are of the form $(x, m) \mapsto\left(x,\left(r_{G} \circ \bar{g}_{i j}\right)(x) m\right)$ where $\bar{g}_{i j} \in \mathrm{M}\left(p\left(\bar{U}_{i} \cap \bar{U}_{j}\right), \bar{G}\right)$.

If an isometry $f: Y \rightarrow Y^{\prime}$ is locally given by $g_{i} \in \mathrm{M}\left(p\left(U_{i}\right), G\right)$ then one similarly checks that the isometry $\bar{f}: \bar{Y} \rightarrow \bar{Y}^{\prime}$ is locally given by $r_{G} \circ \bar{g}_{i} \in \mathrm{M}\left(p\left(\bar{U}_{i}\right), G\right)$.

Example 6.20. In the case of 1|1-dimensional Euclidean cs-structures from Example 6.16 , we can define the real structure $r_{\mathbb{M}}$ on $\mathbb{M}=\mathbb{E}^{1 \mid 1}=\mathbb{R}_{\mathrm{cs}}^{1 \mid 1}$ as in Definition 6.18. This also defines the real structure on the translational part of

$$
G=\operatorname{Iso}\left(\mathbb{E}^{1 \mid 1}\right)=\mathbb{R}_{\mathrm{cs}}^{1 \mid 1} \rtimes \mathbb{Z} / 4
$$

If we think of $\mathbb{Z} / 4$ as the 4 th roots of unity inside $\mathbb{C}$ then the usual real structure on $\mathbb{C}$ induces one on $\mathbb{Z} / 4$. On the pin generator pg, this real structure is $r_{G}(\mathrm{pg}):=\mathrm{pg}^{-1}$ which means that it permutes the sheets, even of the reduced manifold $\mathbb{R} \rtimes \mathbb{Z} / 4$. This permutation is necessary in order to make $\left(r_{G}, r_{\mathbb{M}}\right)$ into a real structure on the local model $(G, \mathbb{M})$ for Euclidean cs-manifolds.

6.4. Fibred Euclidean bordism categories. In this subsection we define the (family) Euclidean bordism category $d$-EB $\rightarrow$ Man and its generalizations. These are symmetric monoidal categories (Grothendieck) fibred over the site Man of smooth manifolds, compare [29] or [11].

Definition 6.21. An object in $d$-EB is given by $\left(S, Y, Y^{\mathrm{c}}, Y^{ \pm}\right)$, where $S$ is a smooth manifold, $Y \rightarrow S$ is a family of $d$-dimensional Euclidean manifolds and $Y^{\mathrm{c}} \subset Y$ is a smooth codimension 1 submanifold such that the restriction of $p$ to 
$Y^{\mathrm{c}}$ is proper. This assumption is a family version of our previous assumption in Definition 6.4 that $Y^{\mathrm{c}}$ is compact, since it reduces to that assumption for $S=\mathrm{pt}$. Also part of the data is the decomposition of $Y \backslash Y^{\mathrm{c}}$ as the disjoint union of two open subsets $Y^{ \pm}$, both of which contain $Y^{\mathrm{c}}$ in their closure.

A morphism $\Sigma$ in $d$-EB, lying over a smooth map $f: S \rightarrow S^{\prime}$ of manifolds, is defined as follows: First fix the source $\left(S, Y_{0}\right)$ and target $\left(S,{ }^{\prime} Y^{\prime}\right)$ of $\Sigma$ which are objects as above. Then pull back the target to get another object $\left(S, f^{*}\left(Y^{\prime}\right)\right)$ over $S$ which we denote by $\left(S, Y_{1}\right)$. Then $\Sigma=\left(\Sigma, p_{\Sigma}, i_{0}, i_{1}\right)$ contains the following additional data:

- a family of $d$-dimensional Euclidean manifolds $p_{\Sigma}: \Sigma \rightarrow S$

- for $=0,1$, smooth maps $i_{j}: W_{j} \rightarrow \Sigma$ over $S$, where $W_{j} \subset Y_{j}$ are open neighborhoods of the cores $Y_{j}^{\mathrm{c}}$. Letting $i_{j}^{ \pm}: W_{j}^{ \pm} \rightarrow \Sigma$ be the restrictions of $i_{j}$ to $W_{j}^{ \pm}:=W_{j} \cap Y_{j}^{ \pm}$, we require that

(+) $i_{j}^{+}$are isometric embeddings into $\Sigma \backslash i_{1}\left(W_{1}^{-} \cup W_{1}^{\mathrm{c}}\right)$ and

(c) the restriction of $p_{\Sigma}$ to the core $\Sigma^{\mathrm{c}}:=\Sigma \backslash\left(i_{0}\left(W_{0}^{+}\right) \cup i_{1}\left(W_{1}^{-}\right)\right)$is proper.

Two such families of Euclidean bordisms $\Sigma \rightarrow S$ and $\Sigma^{\prime} \rightarrow S$ represent the same morphism in $d$-EB if they are isometric (rel. boundary) over the identity of $S$.

Then $d$-EB $\rightarrow$ Man is a fibred category which has a fibrewise symmetric monoidal structure, simply given by disjoint union (of total spaces $Y$ respectively $\Sigma$ ). It is customary to denote by $d$-EB $\mathrm{EB}_{S}$ the fibre categories of this fibration: For fixed $S \in$ Man, the objects are families $Y \rightarrow S$ and the morphisms are restricted to lie over the identity map of $S$.

Recalling the categories $\mathrm{M}(G, \mathbb{M})$ of families of $(G, \mathbb{M})$-manifolds (and their isometries) from Section 6.3, it is not hard to use the same technique as above to define the family bordism categories $\mathrm{B}(G, \mathbb{M}) \rightarrow \mathrm{M}$. If $\mathrm{M}$ is one of the two possible sites of super manifolds then we note that the properness assumptions on $p: Y^{\mathrm{c}} \rightarrow S$ is only an assumption on the reduced (ordinary) manifolds.

In particular, using Euclidean cs-manifolds of dimension $1 \mid 1$ as defined in Example 6.16 , we get a fibred symmetric monoidal category $1 \mid 1-\mathrm{EB} \rightarrow \mathrm{csM}$.

Definition 6.22. In this paper we shall make an additional positivity assumption on the cores of our Euclidean families: If $Y \rightarrow S$ is a familiy of Euclidean manifolds (representing objects or morphisms in $d$-EB) then we require that the induced map of cores $Y^{\mathrm{c}} \rightarrow S$ is a locally trivial fibre bundle and refer to this as a positive family. Roughly speaking, this means that we only explore the topology on the open part of the moduli space of Euclidean bordisms. We shall denote this positive Euclidean bordism category by $d$-PEB.

Using the same positivity assumption, we get a fibred category $1 \mid 1-\mathrm{PEB} \rightarrow$ csM of positive Euclidean cs-bordisms.

For $d=1$ positivity implies that there are no smooth families that shrink the intervals $I_{t}$ or $L_{t}$ as $t \mapsto 0$. Such families exist for $t>0$ and the morphisms $I_{0}=\mathrm{id}_{\mathrm{pt}}$ and $L_{0}$ also exist but we don't consider the limits $t \mapsto 0$, motivating the use of the word positive. As a consequence, $I_{0}$ and $L_{0}$ represent isolated points in the moduli space of intervals.

This assumption of positivity is related to our choice of the family version of the target category Vect to be that of (locally trivial) vector bundles. In future 
work, we plan to investigate more general family bordism categories (partial compactifications of our open moduli space) mapping to more general sheaves of vector spaces.

The forgetful functor $d$-PEB $\rightarrow$ Man sending $\left(S, Y, Y^{\mathrm{c}}, Y^{ \pm}\right)$to $S$ is a Grothendieck fibration and will be considered as part of the structure of the family bordism category. We will denote by $d$-PEB $S$ the fibre category over the manifold $S$, describing $S$-families of positive Euclidean bordisms.

The disjoint union of total spaces $Y$ gives a (fibrewise) symmetric monoidal structure on this fibration as explained in [11] and [12]. We have the following family version of Theorem 6.11 .

Theorem 6.23. The fibred symmetric monoidal category 1-PEB $\rightarrow$ Man is generated by the object $\mathrm{pt} \in 1-\mathrm{PEB}_{\mathrm{pt}}$ and the morphisms $L_{0} \in 1-\mathrm{PEB} \mathrm{pt}(\mathrm{pt} \amalg \mathrm{pt}, \varnothing)$ and $R \in 1-\mathrm{PEB}_{\mathbb{R}_{>0}}(\varnothing$, pt $\amalg \mathrm{pt})$ subject to the following relations

- $L_{0}$ and $R$ are symmetric as before: $L_{0}=L_{0} \circ \sigma, R=\sigma \circ R$

- The relation $R_{t_{1}+t_{2}}=R_{t_{1}} \circ_{L_{0}} R_{t_{2}}$ shown in (5) holds, interpreted as saying that using the three maps $p_{1}, p_{2}, m: \mathbb{R}_{>0} \times \mathbb{R}_{>0} \rightarrow \mathbb{R}_{>0}$, where $m$ is addition and $\mathbb{R}_{>0}$, one has

$$
p_{1}^{*}(R) \circ_{L_{0}} p_{2}^{*}(R)=m^{*}(R) \in 1-\mathrm{PEB}_{\mathbb{R}_{>0} \times \mathbb{R}_{>0}}(\varnothing, \mathrm{pt} \amalg \mathrm{pt})
$$

We note that $R_{t}$ is obtained from $R$ by pullback via the map pt $\rightarrow \mathbb{R}_{>0}$ with image $t>0$. Theorem 6.23 follows from Theorem 6.11 by an analysis of the topology of the moduli space of intervals, see [12].

As in Corollary 6.12, the theorem has the following important consequence (which can again be used as the definition of the notion of "generators and relations" for fibred symmetric monoidal categories).

Corollary 6.24. For any symmetric monoidal category $\mathrm{C} \rightarrow$ Man, fibred over smooth manifolds, the groupoid of fibred symmetric monoidal functors from 1-PEB to $\mathrm{C}$ (and natural isomorphisms) is equivalent to a certain groupoid $\mathrm{C}^{3}$ :

$$
\operatorname{Fun}_{\operatorname{Man}}^{\otimes}(1-\mathrm{PEB}, \mathrm{C}) \simeq \mathrm{C}^{3}
$$

Here the objects of $\mathrm{C}^{3}$ are triples $(V, \mu, \rho)$ where $V \in \mathrm{C}_{\mathrm{pt}}$ and

$$
\mu \in \mathrm{C}_{\mathrm{pt}}(V \otimes V, \mathbb{1}) \text { respectively } \rho \in \mathrm{C}_{\mathbb{R}_{>0}}(\mathbb{1}, V \otimes V)
$$

are symmetric morphisms in $\mathrm{C}$ subject to the relations $\rho_{t_{1}+t_{2}}=\rho_{t_{1}} \circ_{\mu} \rho_{t_{2}}$ as explained in the theorem above. The morphisms $\mathrm{C}^{3}\left((V, \mu, \rho),\left(V,^{\prime} \mu,^{\prime} \rho^{\prime}\right)\right)$ are isomorphisms in $\mathrm{C}_{\mathrm{pt}}\left(V, V^{\prime}\right)$ for which the obvious diagrams involving $\mu, \mu,{ }^{\prime} \rho, \rho^{\prime}$ commute.

Note that the same result holds without the above restriction to isomorphisms but we chose this formulation because it is the groupoids whose classifying space later leads to the correct homotopy types.

In particular, we can consider as target the fibred category $\mathrm{Fr}_{\mathbb{R}} \rightarrow$ Man of real Fréchet vector bundles over smooth manifolds, equipped with fibrewise projective tensor product. This leads to the following corollary.

Corollary 6.25. The groupoid $\mathrm{Fun}_{\mathrm{Man}}^{\otimes}\left(1-\mathrm{PEB}, \mathrm{Fr}_{\mathbb{R}}\right)$ is equivalent to the groupoid of triples $(V, \mu, \rho)$ where $V$ is a Fréchet space with a symmetric pairing $\mu: V \otimes$ $V \rightarrow \mathbb{R}$ and a smooth symmetric semigroup $\rho: \mathbb{R}_{>0} \rightarrow V \otimes V$. Morphisms are isomorphisms of Fréchet spaces, compatible with the extra structure given by $\mu, \rho$. 
The equivalence is given by assigning to a field theory $E$ the triple $(V, \mu, \rho)$, where

$$
V=E(\mathrm{pt}), \quad \mu=E\left(L_{0}\right) \quad \text { and } \quad \rho=E(R)
$$

Definition 6.26. A 1-dimensional field theory $E \in \mathrm{Fun}_{\mathrm{Man}}^{\otimes}\left(1-\mathrm{PEB}, \mathrm{Fr}_{\mathbb{R}}\right)$ is nonnegative if $\mu(v, w) \geq 0$ for all $v, w \in V$. We call $E$ positive if $V$ is in addition infinite-dimensional and separable and $\mu$ gives an (algebraic) isomorphism from $V$ to its continuous dual space $V^{\prime}$. The full subgroupoid of $\operatorname{Fun}_{\mathrm{Man}}^{\otimes}\left(1-\mathrm{PEB}, \mathrm{Fr}_{\mathbb{R}}\right)$ given by positive field theories is the denoted by 1-PEFT, the groupoid of positive Euclidean field theories of dimension 1.

Remark 6.27. We point out that the notion of a positive Euclidean field theory has two aspects:

(1) the functor $E$ is defined on the positive Euclidean bordism category.

(2) The inner product $\mu=E\left(L_{0}\right)$ is positive in the above sense.

Both these conditions have their own reasons to be called positive and as a (desirable) consequence, we only need one adjective to describe the resulting Euclidean field theory.

In the positive case, Lemma 6.3 implies that $V$ is a real Hilbert space with inner product $\mu$. Therefore, $V \otimes V$ is the space of trace-class operators and we obtain the following final computation from the fact that any two infinite-dimensional, separable Hilbert spaces are isometric.

Corollary 6.28. Let $(H, \mu)$ be an infinite-dimensional separable real Hilbert space. Then the groupoid 1-PEFT of positive Euclidean field theories of dimension 1 is equivalent to the groupoid 1-PEFT $(H)$ of smooth semigroups $\rho_{t}$ of self-adjoint trace-class operators on $H$. The morphisms are isometries of $(H, \mu)$ that are compatible with the semigroups.

We note that in the absence of the positivity condition in Definition 6.22, 1EFTs satisfying the conditions of Definition 6.26 do not exist at all! This comes from the fact that if the semigroup $\rho_{t}=\exp (-t A)$ extends smoothly to $t=0$ then its infinitesimal generator $A$ needs to give a continuous operator on $V$. If $V$ is a Hilbert space then $A$ needs to be bounded and the trace-class condition implies that $A$ and hence all $\rho_{t}$ have finite rank. Taking the limit $t \mapsto 0$ shows that $\rho_{0}=\operatorname{id}_{V}$ and hence $V$ is finite-dimensional.

Our assumption that the EFT is only defined on positive bordisms in 1-PEB implies in particular that $\rho_{0}$ does not have to be the identity on $V$, it only has to be a projection operator. We shall refer to the kernel of $\rho_{0}$ as the $\infty$-Eigenspace of $A$.

In [12] we shall study general 1-EFTs, i.e.,. the groupoid $\operatorname{Fun}_{\mathrm{Man}}^{\otimes}\left(1-\mathrm{EB}, \mathrm{Fr}_{\mathbb{R}}\right)$, so that we have to allow $V$ to be a Fréchet space like $C^{\infty}\left(S^{1}\right)$. Then the pairing $\mu$ may only induce an injection $V \hookrightarrow V^{\prime}$ and may even be indefinite. The advantage of using $C^{\infty}$, rather than $L^{2}$-functions, is that the infinitesimal generator $A$ can be unbounded but continuous on $V$, for example if $A$ comes from the differentiation operator. We believe that this general class of 1-EFTs still leads to a homotopy equivalent classifying space if one uses the construction from the next section, see Remark 6.30. 
6.5. Internal groupoids of smooth field theories. In this section we explain how groupoids of smooth field theories can be naturally equipped with a topology so that we may consider the corresponding classifying spaces. More precisely, if $\mathrm{G}$ is one of these groupoids, we shall naturally define a new groupoid $\operatorname{Man}(M, \mathrm{G})$ for every manifold $M \in$ Man which serves as the groupoid of smooth maps from $M$ to $\mathrm{G}$. In fact, these groupoids will fit together to a category $\underline{G} \rightarrow$ Man fibred in groupoids. In all cases at hand, we will show that $\underline{\mathrm{G}}$ is equivalent to a fibred groupoid coming from a contravariant functor from manifolds to small groupoids:

$$
\mathrm{sG}: \mathrm{Man}^{\mathrm{op}} \rightarrow \mathrm{sGrp}
$$

In particular, this functor sG has a well defined classifying space, obtained from realizing the simplicial space

$$
\Delta^{\mathrm{op}} \rightarrow \mathrm{Man}^{\mathrm{op}} \stackrel{\mathrm{sG}}{\longrightarrow} \rightarrow \mathrm{sGrp} \stackrel{\text { |.| }}{\rightarrow} \text { Top }
$$

We shall denote this classifying space simply by $|G|$ to emphasize the naturality of the construction of $\underline{G}$ and since the choice of $\mathrm{sG}$ cannot alter the homotopy type of this classifying space. We shall give this construction in great generality in order to

(1) express its naturality,

(2) be able to apply it to supersymmetric EFTs,

(3) use it in dimensions $d>1$ in future papers.

The main goal of the current Section 6 is the following result:

Theorem 6.29. In the notation of this and the following subsection, the groupoids 1-PEFT and 1|1-PEFT of positive Euclidean field theories of dimensions 1 respectively $1 \mid 1$ have classifying spaces whose homotopy type is given by:

$$
|1-\mathrm{PEFT}| \simeq \mathrm{pt} \quad \text { and } \quad|(1 \mid 1)-\mathrm{PEFT}| \simeq B O \times \mathbb{Z}
$$

Remark 6.30. In [12] we ignore all positivity assumptions and work with general sheaves of Fréchet spaces to obtain the groupoids 1-EFT and 1|1-EFT. The construction of this section applies to obtain the corresponding classifying spaces and we conjecture that this does not change homotopy types:

$$
|1-\mathrm{EFT}| \simeq|1-\mathrm{PEFT}| \text { and }|(1 \mid 1)-\mathrm{EFT}| \simeq|(1 \mid 1)-\mathrm{PEFT}|
$$

Note however, that applying our current construction in dimensions 0 and $0 \mid 1$ leads to contractible classifying spaces

$$
|0-\mathrm{EFT}| \simeq|(0 \mid 1)-\mathrm{EFT}| \simeq \mathrm{pt}
$$

This is the reason why we worked with field theories over a manifold $X$ in [11]. We proved that this approach leads to the correct homotopy type for de Rham cohomology to appear (in the twisted case) by using simplices in place of $X$. We believe that this more difficult construction gives the correct homotopy type in all dimensions and view our current method as a shortcut for $K$-theory, i.e., for dimension 1|1. We are uncertain whether this shortcut also works in the most interesting dimension $2 \mid 1$.

The groupoids $G$ for which we shall construct a classifying space $|G|$ will be of the following form: Let $S$ be a base category, in this paper $S=$ Man or SMan. We require that $\mathrm{S}$ has finite products and we fix a functor Man $\rightarrow \mathrm{S}$. Our notation will not reflect this functor, i.e., we will just think of manifolds as objects in $\mathrm{S}$ (which is certainly correct in the two cases at hand). Let $\mathrm{B}$ and $\mathrm{V}$ be two categories fibred 
over S, where B will later be some bordism category and $\mathrm{V}$ some category of vector bundles. Then we consider the groupoid of fibred functors

$$
\mathrm{G}=\operatorname{Fun}_{\mathrm{S}}(\mathrm{B}, \mathrm{V})
$$

where the morphisms are natural isomorphisms over S. In our applications, B and $\mathrm{V}$ will actually be symmetric monoidal fibred categories and the functors will be assumed to be symmetric monoidal.

Definition 6.31. The category $\underline{G} \rightarrow S$ fibred in groupoids is defined as the inner Hom in fibred categories. Its fibre $\underline{\mathrm{G}}_{S}$ over an object $S \in \mathrm{S}$ is given by

$$
\underline{\mathrm{G}}_{S}=\underline{\mathrm{Fun}}_{\mathrm{S}}(\mathrm{B}, \mathrm{V})_{S}=\operatorname{Fun}_{\mathrm{S}}(\mathrm{B}, \mathrm{V}(S))
$$

where $\mathrm{V}(S)=\underline{\mathrm{Fun}}_{S}(\underline{S}, \mathrm{~V})$ is the fibred category with fibres $\mathrm{V}(S)_{T}:=\mathrm{V}_{S \times T}$. Here all functor categories are considered as groupoids, i.e., we only allow natural isomorphisms, not all natural transformations, as morphisms.

Remark 6.32. This definition is a certain version of inner Hom, where the variable $S$ is built in on the right hand side. This implies good functoriality properties and applications of Corollary 6.24 in the case $B=1-P E B$. We note that in general there are equivalences of categories

$$
\operatorname{Fun}_{\mathrm{S}}\left(\mathrm{B}, \underline{\operatorname{Fun}}_{\mathrm{S}}(\underline{S}, \mathrm{~V})\right) \simeq \operatorname{Fun}_{\mathrm{S}}(\mathrm{B} \times \underline{S}, \mathrm{~V}) \simeq \underline{\operatorname{Fun}}_{\mathrm{S}}(\mathrm{B}, \mathrm{V})_{S}
$$

because

$$
\mathrm{V}(S)_{T}=\mathrm{V}_{S \times T} \simeq \operatorname{Fun}_{\mathrm{S}}(\underline{S \times T}, \mathrm{~V}) \simeq \mathrm{Fun}_{\mathrm{S}}(\underline{S}, \mathrm{~V})_{T}
$$

As an example, let's take the relevant fibred categories from the previous section, i.e.,

$$
\mathrm{S}=\text { Man, } \quad \mathrm{B}=1 \text {-PEB } \text { and } \mathrm{V}=\mathrm{Fr}_{\mathbb{R}}
$$

Then we can apply Corollary 6.24 to the fibred category $C=\operatorname{Fr}_{\mathbb{R}}(S)$ for any manifold $S$. We obtain an equivalence of groupoids

$$
\underline{\operatorname{Fun}}_{\operatorname{Man}}^{\otimes}\left(1-\mathrm{PEB}, \operatorname{Fr}_{\mathbb{R}}\right)_{S} \simeq \operatorname{Fr}_{\mathbb{R}}(S)^{3}
$$

where the objects of the groupoid $\operatorname{Fr}_{\mathbb{R}}(S)^{3}$ are triples $(V, \mu, \rho)$. Here $V$ is a Fréchet bundle over $S$ with a symmetric pairing $\mu$ and symmetric semigroup $\rho$.

We now restrict the functors to be positive, in the (family version) sense of Definition 6.26 and obtain (full) subgroupoids

$$
\underline{1-P E F T}_{S} \subset \underline{\text { Fun }}_{\text {Man }}^{\otimes}\left(1-\mathrm{PEB}, \mathrm{Fr}_{\mathbb{R}}\right)_{S}
$$

The above equivalence takes this subgroupoid to the category of triples $(V, \mu, \rho)$ with $V$ is a Hilbert bundle over $S$, fibrewise inner product $\mu$ and self-adjoint semigroup $\rho$.

By the contractibility of the orthogonal group $O(H)$, this category is equivalent to the one where $V$ is the trivial bundle $H \times S$ and $\mu$ is constant. Then $\rho: S \rightarrow$ $\mathcal{S G O}^{\mathrm{TC}}(H)$ is a smooth map into all self-adjoint semigroup homomorphisms $\mathbb{R}_{>0} \rightarrow$ $H \otimes H$, where the right hand side denotes the projective tensor product, isomorphic to the space of trace-class operators $\mathrm{TC}(H)$.

Smoothness means that the adjoint of $\rho$, the map $S \times \mathbb{R}_{>0} \rightarrow H \otimes H$, is smooth. It is self-adjoint and has the semigroup property for each fixed $s \in S$. 
Remark 6.33. The notation 'semigroup of operators' $\mathcal{S} \mathcal{G O}^{\mathrm{TC}}$ is also used in Proposition 5.16 where there is an additional index $n$, expressing the fact that the Hilbert space in question is graded and has a $C_{n}$-action. Here we have no grading (until we get to supersymmetric field theories) and no $C_{n}$-action (until we discuss twisted field theories of degree $n$ ) but we prefer to keep the infinite-dimensional separable Hilbert space $H$ in the notation.

Summarizing, we get

Corollary 6.34. The fibred category 1 -PEFT $\rightarrow$ Man is equivalent to the one coming from the functor into small groupoids:

$$
\mathcal{S G O}^{\mathrm{TC}}(H) / O(H): \mathrm{Man}^{\text {op }} \rightarrow \mathrm{sGrp}
$$

This is actually a quotient fibration, i.e., the value of this functor at a manifold $S$ is the transport groupoid for the action of $C^{\infty}(S, O(H))$ on $C^{\infty}\left(S, \mathcal{S G O}^{\mathrm{TC}}(H)\right)$.

The last step in this discussion is the computation of the classifying space of this quotient fibration. As explained at the beginning of this subsection, it is defined by using (extended) $k$-simplices as manifolds $S$ above to obtain a small simplicial groupoid that can be geometrically realized to get $|1-\mathrm{PEFT}|$.

This realization means that one forms a bisimplicial set by taking the nerves of the groupoids involved. Then one has the choice of realizing this bisimplicial set in the two possible orders. If we first realize the original simplicial direction we get a simplicial space that is the nerve of an internal groupoid in Top with objects respectively morphisms given by the realizations of the simplicial sets

$$
[k] \mapsto C^{\infty}\left(\Delta^{k}, O(H)\right) \quad \text { respectively }[k] \mapsto C^{\infty}\left(\Delta^{k}, \mathcal{S G O}^{\mathrm{TC}}(H)\right)
$$

By a version of the smooth approximation theorem, these simplicial sets have the same homotopy type as the singular simplicial sets of $O(H)$, respectively $\mathcal{S G O}^{\mathrm{TC}}(H)$ (which are formed by replacing $C^{\infty}$ by $C^{0}$ in the above equations). Both of these spaces have the homotopy type of $\mathrm{CW}$-complexes and hence the realizations of this singular simplicial sets are homotopy equivalent to the original spaces $O(H)$ respectively $\mathcal{S G O}^{\mathrm{TC}}(H)$. The nerve of this internal (transport) groupoid in Top then has realization $|1-\mathrm{PEFT}|$, proving the first part of the following result.

Theorem 6.35. The classifying space of the groupoid 1-PEFT of positive 1EFTs has the homotopy type

$$
|1-\mathrm{PEFT}| \simeq\left|\mathcal{S} \mathcal{G O} \mathcal{O}^{\mathrm{TC}}(H) / O(H)\right| \simeq \mathcal{S} \mathcal{G O} \mathcal{O}^{\mathrm{TC}}(H) \simeq \mathrm{pt}
$$

Proof. The second homotopy equivalence follow from the contractibility of $O(H)$. It remains to show that the space $\mathcal{S G O}^{\mathrm{TC}}(H)$ of self-adjoint trace-class semigroups in $H$ is contractible. The graded analogue was discussed at length in Section 5, see particularly Propositions 5.12 and 5.16. Therefore we shall be fairly brief in this argument. First recall that the zero semigroup $\rho_{t} \equiv 0$ serves as the basepoint in $\mathcal{S G O}^{\mathrm{TC}}(H)$. It corresponds to all eigenspaces of the infinitesimal generator $A, \rho_{t}=\exp (-t A)$, being at $\infty$. A contraction

$$
H: \mathcal{S G O}^{\mathrm{TC}}(H) \times[1, \infty] \rightarrow \mathcal{S G O}^{\mathrm{TC}}(H)
$$

to this basepoint is then given by the formula $H\left(\rho_{t}, s\right):=\rho_{s \cdot t}$. This has infinitesimal generator $s \cdot A$ and, looking at its eigenspaces, we see that they move to $\infty$ as $s \mapsto \infty$. 
Remark 6.36. By looking at the eigenspaces of infinitesimal generators, the set $\mathcal{S G O}^{\text {fin }}(H)$ can be identified with Segal's configuration space $F((-\infty,+\infty])$ discussed in the introduction. This comes from the fact that eigenspaces can move exactly to $+\infty$ in $\mathcal{S G O}^{\text {fin }}(H)$ because $\exp (-s A)$ becomes zero as $s \mapsto+\infty$ and unbounded as $s \mapsto-\infty$. Our contraction of $\mathcal{S G O}^{\mathrm{fin}}(H)$ in the proof above is then just the configuration space version of the contraction of $(-\infty,+\infty]$ to the point $+\infty$.

6.6. Proof of part (6) of our Main Theorem. In the remaining parts of Section 6, we shall redo all of the above considerations for super manifolds to obtain the classifying space of positive 1|1-dimensional Euclidian field theories. It will turn out that it has an interesting homotopy type, rather than being contractible as above. We need the following steps:

Step (1) Recall the Euclidean cs-bordism category 1|1-EB and its positive subcategory $1 \mid 1-P E B$.

Step (2) Find generators and relations for 1|1-PEB.

Step (3) Identify the resulting category $1 \mid 1-\mathrm{PEFT} \rightarrow$ Man fibred in groupoids with

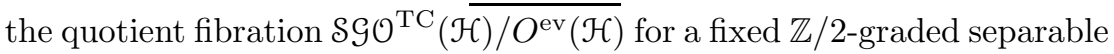
Hilbert space $\mathcal{H}$ such that the even and odd parts are infinite-dimensional.

Step (4) Compute the homotopy type of the classifying space for supersymmetric positive $1 \mid 1-E F T s$

$$
|(1 \mid 1)-\mathrm{PEFT}| \simeq\left|\mathcal{S G O} \mathcal{O}^{\mathrm{TC}}(\mathcal{H}) / O^{\mathrm{ev}}(\mathcal{H})\right| \simeq \mathcal{S} \mathcal{S O}{ }^{\mathrm{TC}}(\mathcal{H}) \simeq B O \times \mathbb{Z}
$$

Similarly to the discussion around Theorem 6.35, step (4) follows from step (3) by Propositions 5.12 and 5.16.

Step (1) was explained in Section 6.4, where we defined the family bordism categories $\mathrm{B}(G, \mathbb{M}) \rightarrow \mathrm{M}$ for arbitrary $(G, \mathbb{M})$-structures on the site $\mathrm{M}$. The case of Euclidean structures in arbitrary dimension $d \mid \delta$ is explained in [29], for dimension $1 \mid 1$ see Example 6.16 and Definition 6.21.

Step (2) is then a computation of the relevant super-moduli spaces of 0 - and 1-handles and will be carried out in the next subsections. In the remainder of this subsection, we'll use the following corollary to Theorem 6.48 of to finish step (3) of the current discussion.

Corollary 6.37. Let $\mathcal{H}$ be a separable real Hilbert space, graded in a way such that the even and odd parts are both infinite-dimensional. Then the groupoid 1|1-PEFT of positive Euclidean field theories of dimension $1 \mid 1$ is equivalent to the groupoid $1 \mid 1-\mathrm{PEFT}(\mathcal{H})$ of super-semigroups $\varrho_{t, \theta}$ of even self-adjoint trace-class operators on $\mathcal{H}$. The morphisms are even isometries of $\mathcal{H}$ that are compatible with the semigroups.

Corollary 6.38. The fibred category $1 \mid 1-\mathrm{PEFT} \rightarrow$ Man is equivalent to the one coming from the quotient fibration

$$
\mathcal{S G O}^{\mathrm{TC}}(\mathcal{H}) / O^{\mathrm{ev}}(\mathcal{H}): \operatorname{Man}^{\text {op }} \rightarrow \mathrm{sGrp}
$$

The same computation of the classifying space of this quotient fibration as in Theorem 6.35 shows that it has the homotopy type of $\mathcal{S G O}^{\mathrm{TC}}(\mathcal{H})$. This is the space denoted by $\mathcal{S} \mathcal{G O}_{0}^{\mathrm{TC}}$ in Proposition 5.16, where it was shown that its homotopy type is that of

$$
|(1 \mid 1)-\mathrm{PEFT}| \simeq \mathcal{S G \mathcal { O } _ { 0 }} \approx \mathrm{Conf}_{0} \simeq B O \times \mathbb{Z}
$$


This finishes the proof of part 6 of our Main Theorem in the introduction, modulo Theorem 6.48.

6.7. Generators and relations for 1|1-PEB. First we note that any super or cs-manifold has an involution that negates its odd coordinate. We shall refer to this as the pin flip, written fl. If $Y$ has a $(G, \mathbb{M})$-structure, one may ask whether $\mathrm{fl}_{Y}$ is an isometry. It turns out that this is indeed the case if $f_{\mathbb{M}}$ comes from an element $g \in G$ in the sense explained at the end of Definition 6.13. This is true in the example of 1|1-dimensional Euclidean cs-manifolds, where $\mathrm{f}_{\mathbb{M}}$ is the square of the pin generator pg, see Example 6.16.

The following object $\mathrm{sp} \in 1 \mid 1-\mathrm{EB}$ will be referred to as the super-point

$$
\mathrm{sp}:=\left(\mathrm{sp}, \mathrm{sp}^{\mathrm{c}}, \mathrm{sp}^{ \pm}\right):=\left(\mathbb{E}^{1 \mid 1}, \mathbb{R}^{0 \mid 1}, \mathbb{R}_{ \pm}^{1 \mid 1}\right)=\mathrm{pt} \times \mathbb{R}^{0 \mid 1}
$$

Note that the core is again a codimension 1 submanifold and for the following lemma it is essential that an automorphism of the super-point is required to preserve it.

Lemma 6.39. The (inner) isometry group Iso(sp) of the super-point is $\mathbb{Z} / 2$, generated by fl.

Proof. By definition, the isometry group of our super Euclidean space $\mathbb{E}^{1 \mid 1}=$ $\mathbb{R}^{1 \mid 1}$ is

$$
\underline{\mathrm{Iso}}\left(\mathbb{E}^{1 \mid 1}\right)=\mathbb{R}^{1 \mid 1} \rtimes \mathbb{Z} / 4
$$

and we need to decide which of these isometries preserve the additional data $\mathrm{sp}^{\mathrm{c}}$ and $\mathrm{sp}^{ \pm}$. The core $\mathrm{sp}^{\mathrm{c}}$ of $\mathrm{sp}$ is given by coordinates $(0, \eta)$. Hence none of the translations

$$
T_{t, \theta}(0, \eta)=(t+\theta \eta, \theta+\eta)
$$

preserve the core. The pin generator $\operatorname{pg}(t, \theta)=(-t,-i \theta)$ does preserve the core but only its square preserves the parts $\mathrm{sp}^{ \pm}$of the super point. This is exactly the pin flip fl.

Remark 6.40. There is another object in 1|1-EB that looks just like the above super-point, with the same ambient manifold and core but where the \pm -parts are interchanged. The pin generator is an isometry from this new object to sp and hence we don't need to consider this as an extra generator. In the oriented bordism category, these two objects are not isomorphic.

To obtain interesting families of Euclidean cs-manifolds over a fixed cs-manifold $S$, we start with a function

$$
f \in C^{\infty}(S) \cong \operatorname{csM}\left(S, \mathbb{R}^{1 \mid 1}\right) \quad \text { such that } f_{\text {red }}: S_{\text {red }} \rightarrow \mathbb{R}_{\text {red }}^{1 \mid 1}=\mathbb{R} \text { satisfies } f_{\text {red }}>0 .
$$

Then we can use the super group structure $m$ on $\mathbb{R}^{1 \mid 1}$ given in Definition 5.3 to define the translation map

$$
T_{f}: S \times \mathbb{R}^{1 \mid 1} \stackrel{\text { id } \times f \times \text { id }}{\longrightarrow} S \times \mathbb{R}^{1 \mid 1} \times \mathbb{R}^{1 \mid 1} \stackrel{\text { id } \times m}{\longrightarrow} S \times \mathbb{R}^{1 \mid 1}
$$

Let $\Sigma:=S \times \mathbb{E}^{1 \mid 1}$ with $i_{\text {out }}$ the identity map $S \times \operatorname{sp} \rightarrow \Sigma$ and $i_{\text {in }}$ be given by $T_{f}$.

Definition 6.41. For any cs-manifold $S$, write $f \in C^{\infty}(S)$ as $f=t+\theta$ where $t$ and $\theta$ are even respectively odd functions on $S$ and assume that $f_{\text {red }}=t_{\text {red }}>0$. Then the above morphism $\left(\Sigma, i_{\text {in }}, i_{\text {out }}\right)$ is called the super-interval of length $(t, \theta)$, written as

$$
I_{t, \theta} \in 1 \mid 1-\mathrm{PEB}_{S}(S \times \mathrm{sp}, S \times \mathrm{sp}) .
$$


It lies over the identity map of $S$ in csM. There are two close cousins also lying over the identity of $S$, the left, respectively right, elbow of length $(t, \theta)$, written as $R_{t, \theta} \in 1 \mid 1-\mathrm{PEB}_{S}(\varnothing, S \times(\mathrm{sp} \amalg \mathrm{sp})) \quad$ respectively $L_{t, \theta} \in 1 \mid 1-\mathrm{PEB}_{S}(S \times(\mathrm{sp} \amalg \mathrm{sp}, \varnothing))$.

These are defined like $I_{t, \theta}$ with one exception, namely the pin generator pg has to enter the discussion (it does not depend on the parameters in $S$ ). For the left elbow, $i_{\text {out }}$ is empty and $i_{\text {in }}$ is the disjoint union of $\mathrm{pg}$ and the translated embedding $T_{f}$. For the right elbow, $i_{\text {in }}$ is empty and $i_{\text {out }}$ is the disjoint union of the identity and $T_{f}$ precomposed by pg.

We note that as before, the left elbow $L_{0}$ also exists, except that it's not related smoothly to the above family. The above definitions are direct generalizations of bosonic families which we didn't explain in such detail. In particular, the use of the pin generator gives on the reduced part a reflection that needed to be used to parametrize one of the boundary points of $R_{t}$ in (5).

Let's spell out the last definition in the universal case $S=\mathbb{R}_{>0}^{1 \mid 1}$ and $f=\operatorname{id}_{S}$ where the right elbow is just denoted by $R=R_{t, \theta}$. Then $i_{\text {out }}$ is the disjoint union of the identity id: $S \times \mathrm{sp} \rightarrow S \times \mathbb{E}^{1 \mid 1}$ and the isometry

$$
S \times \mathrm{sp} \rightarrow S \times \mathbb{E}^{1 \mid 1}, \quad(t, \theta, y, \eta) \stackrel{p g}{\longmapsto}(t, \theta,-y,-i \eta) \mapsto(t-y-i \theta \eta, \theta-i \eta)
$$

With this convention, one can easily check the super-semigroup relation (4) for $R$ in Theorem 6.42 below. The symmetry condition for $L_{0}$ turns into relation (2):

$$
L_{0} \circ \sigma=L_{0} \circ\left({\text { fl } \amalg i d_{\text {sp }}}\right)
$$

where the right-hand side is $L_{0}$ pre-composed by an isometry of two super-points. This follows from the fact that one has to use the pin generator as the isometry that exchanges the two boundary points of $L_{0}$ which has the claimed consequence for the parametrization of the boundaries. The complete list of generators and relations is as follows. Here $\sigma$ denotes the braiding symmetry of the symmetric monoidal category $1 \mid 1$-PEB.

Theorem 6.42. The fibred symmetric monoidal category $1 \mid 1-\mathrm{PEB} \rightarrow \mathrm{csM}$ is generated by the super point $\mathrm{sp} \in 1 \mid 1-\mathrm{PEB}_{\mathrm{pt}}$, its pin flip $\mathrm{fl}$ and morphisms $L_{0} \in$ $1 \mid 1-\mathrm{PEB}_{\mathrm{pt}}(\mathrm{sp} \amalg \mathrm{sp}, \varnothing)$ respectively $R \in 1 \mid 1-\mathrm{PEB}_{\mathbb{R}_{>0}^{111}}(\varnothing, \mathrm{sp} \amalg \mathrm{sp})$. These are subject to the following relations

(1) $\mathrm{fl}^{2}=\mathrm{id}_{\mathrm{sp}}$

(2) $L_{0} \circ(\mathrm{fl} \amalg \mathrm{fl})=L_{0}$ and $L_{0} \circ(\mathrm{fl} \amalg \mathrm{id})=L_{0} \circ \sigma$

(3) (fl $\amalg \mathrm{fl}) \circ R_{t, \theta}=R_{t,-\theta}$ and (fl $\left.\amalg \mathrm{id}\right) \circ R_{t, i \theta}=\sigma \circ R_{t, \theta}$

(4) $R_{\left(t_{1}+t_{2}+\theta_{1} \theta_{2}, \theta_{1}+\theta_{2}\right)}=R_{\left(t_{1}, \theta_{1}\right)}{ }^{\circ} L_{0} R_{\left(t_{2}, \theta_{2}\right)}$

The last relation is pictured in (5) and says that $R$ is a super-semigroup. This is made precise by using the three maps $p_{1}, p_{2}, m: \mathbb{R}_{>0}^{1 \mid 1} \times \mathbb{R}_{>0}^{1 \mid 1} \rightarrow \mathbb{R}_{>0}^{1 \mid 1}$. Here $m$ is multiplication from Definition 5.3, restricted to $\mathbb{R}_{>0}^{1 \mid 1}:=(0, \infty) \times \mathbb{R}^{0 \mid 1}$, and the precise relation is

$$
p_{1}^{*}(R) \circ L_{0} p_{2}^{*}(R)=m^{*}(R) \in 1 \mid 1-\mathrm{PEB}_{\mathbb{R}_{>0}^{1 \mid 1} \times \mathbb{R}_{>0}^{1 \mid 1}}(\varnothing, \mathrm{sp} \amalg \mathrm{sp})
$$

The proof of Theorem 6.42 will be given in [12], the verification of the above relations (1)-(4) being the first (easy) step. The other steps are the same as in Theorem 6.11, except that at the end of the argument one now needs to compute the relevant cs-moduli spaces of cs-intervals. This is done by a cs-version of the 
developing map, compare [30]. It is used to construct global $(G, \mathbb{M})$-charts for rigid geometries on simply connected manifolds. It then follows that cs-intervals can be embedded isometrically into $\mathbb{E}^{1 \mid 1}$ and that the whole information is contained in the parametrization of one boundary point.

As in Corollary 6.24, this theorem has the following important consequence (which can again be used as the definition of the notion of "generators and relations" for fibred symmetric monoidal categories over csM).

Corollary 6.43. For any fibred symmetric monoidal category $\mathrm{C} \rightarrow \mathrm{csM}$, the groupoid of fibred symmetric monoidal functors from 1|1-PEB to C (and natural isomorphisms) is equivalent to a certain groupoid $\mathrm{C}^{4}$ :

$$
\operatorname{Fun}_{\mathrm{csM}}^{\otimes}(1 \mid 1-\mathrm{PEB}, \mathrm{C}) \simeq \mathrm{C}^{4}
$$

Here the objects of $\mathrm{C}^{4}$ are quadruples $\left(V, \alpha, \mu_{0}, \rho\right)$ where $V \in \mathrm{C}_{\mathrm{pt}}$ and

$$
\alpha \in \mathrm{C}_{\mathrm{pt}}(V, V), \quad \mu_{0} \in \mathrm{C}_{\mathrm{pt}}(V \otimes V, \mathbb{1}) \quad \text { and } \quad \rho \in \mathrm{C}_{\mathbb{R}_{>0}^{1 \mid 1}}(\mathbb{1}, V \otimes V)
$$

are morphisms in $\mathrm{C}$ subject to the relations (1) -(4) given in the theorem above. The morphisms in $\mathrm{C}^{4}$ are isomorphisms in $\mathrm{C}_{\mathrm{pt}}\left(V, V^{\prime}\right)$ for which the obvious diagrams commute. Note that we didn't need to restrict to isomorphisms but we chose to do so in order to later get the correct homotopy type for the relevant classifying space.

In particular, we can consider as target the fibred category SFr $\rightarrow$ csM of complex Fréchet super vector bundles over cs-manifolds, equipped with fibrewise graded projective tensor product. The braiding symmetry comes with the usual sign in this setting. Note also that the pin flip $f$ is taken by any functor $E$ to a grading $E(\mathrm{fl})$ of the (already graded) Fréchet space $V=E(\mathrm{sp})$.

Definition 6.44. We shall say that $E$ satisfies the spin-statistics relation if $E$ (fl) equals the given grading on $V$. We shall write $E \in$ Fun $^{\otimes, s s}$ for such functors.

Remark 6.45. We initially used the pin flip to induce the $\mathbb{Z} / 2$-grading on the vector spaces $E(\mathrm{sp})$ for any EFT $E$. This leads to a different category of (positive) $1 \mid 1-E F T s$ whose classifying space we believe also has the correct homotopy type $B O \times \mathbb{Z}$. Moreover, the tensor product of EFTs gives an $\mathrm{H}$-space structure on this classifying space. However, the induced braiding symmetry is not the one that extends to degree $n$ twists because it comes from the unsigned flip on the category of $\mathbb{Z} / 2$-graded vector spaces. The reason for this is that the Clifford algebras $C_{n}$ only allow graded algebra isomorphisms

$$
C_{n} \otimes C_{m} \cong C_{n+m}
$$

if one uses the signed flip on the category of graded vector spaces, in fact this symmetric monoidal category is by definition that of super vector spaces. Recall that a braiding of a monoidal category is needed in order to give the tensor product of two monoidal objects the structure of a monoidal object. Different braidings, like the unsigned flip versus the signed flip in the monoidal category of graded vector spaces, lead to different notions of monoidal objects. As a consequence, EFTs can only lead to the ring spectrum $K O$ if they naturally lead to the signed flip. We do not see a better way than to introduce this braiding on the target category of super Fréchet spaces SFr to begin with.

Using the spin-statistics relation leads to the following corollary. 
Corollary 6.46. The groupoid $\mathrm{Fun}_{\mathrm{csM}}^{\otimes, s s}(1 \mid 1-\mathrm{PEB}, \mathrm{SFr})$ is equivalent to the groupoid of triples $\left(V, \mu_{0}, \rho\right)$ where $V$ is a graded Fréchet space with an even pairing $\mu_{0}: V \otimes V \rightarrow \mathbb{C}$ that is symmetric in the naive sense:

$$
\mu_{0}(v, w)=\mu_{0}(w, v) \quad \text { for all } v, w \in V .
$$

Moreover, $\rho: \mathbb{R}_{>0}^{1 \mid 1} \rightarrow V \otimes V$ is a morphism of generalized cs-semigroups, as in Definition 5.7, with respect to the composition on $V \otimes V$ induced by $\mu_{0}$. In addition, $\rho$ satisfies the following symmetry condition with respect to the decomposition of $V \otimes V$ into the four blocks $V^{\mathrm{ev}} \otimes V^{\mathrm{ev}}$, etc. given by the grading of $V:$

$$
\rho_{t, \theta}=\left(\begin{array}{cc}
A_{t}^{0} & i \theta \tau B_{t} \\
\theta B_{t} & A_{t}^{1}
\end{array}\right)
$$

where $A^{0}: \mathbb{R}_{+} \rightarrow V^{\mathrm{ev}} \otimes V^{\mathrm{ev}}, A^{1}: \mathbb{R}_{+} \rightarrow V^{\text {odd }} \otimes V^{\text {odd }}$ and $B: \mathbb{R}_{+} \rightarrow V^{\mathrm{ev}} \otimes V^{\text {odd }}$ are smooth maps such that $A^{0}$ and $A^{1}$ are symmetric in the naive sense. Moreover, $\tau: V^{\mathrm{ev}} \otimes V^{\text {odd }} \rightarrow V^{\text {odd }} \otimes V^{\mathrm{ev}}$ is the isomorphism given by $u \otimes v \mapsto v \otimes u$.

Morphisms in this groupoid are isomorphisms of Fréchet spaces, compatible with the extra structure given by $\mu_{0}, \rho$.

It is important to observe that $\mu_{0}$ is $\mathbb{C}$-bilinear and hence we cannot relate this result directly to Hilbert spaces. In the next subsection we shall introduce a notion of real Euclidean field theories for which we obtain an inner product on a real Hilbert space.

Proof of Corollary 6.46. Let's define $V:=E(\mathrm{pt}), \rho:=E(R)$ and $\mu_{0}:=$ $E\left(L_{0}\right)$. By the spin-statistics relation, the grading on $V$ is given by $\alpha=E(\mathrm{fl})$. Therefore, the relations in (2) of Theorem 6.42 say that $\mu_{0}$ is even and satisfies for all homogeneous vectors $v, w \in V$

$$
\mu_{0}(\alpha(v), w)=(-1)^{|v \|| w \mid} \mu_{0}(w, v)
$$

If $v$ and $w$ have different parity, both sides of this equation vanish since $\mu_{0}$ is even. If both vectors are even then it just gives naive symmetry. If both vectors are odd, then both sides pick up a minus sign, and hence again one gets the naive symmetry as claimed.

The next step is to observe that the first relation is (3) of Theorem 6.42 says that $\rho: \mathbb{R}_{>0}^{1 \mid 1} \rightarrow V \otimes V$ is a morphism of generalized cs-semigroups. This follows from the fact that the grading on $\mathbb{R}_{>0}^{1 \mid 1}$ is given by $(t, \theta) \mapsto(t,-\theta)$ whereas it is given by two copies of $\alpha$ on $V \otimes V$. As explained in Definition 5.7, this implies that $\rho$ is of the form $A+\theta B$, where

$$
A: \mathbb{R}_{>0} \rightarrow(V \otimes V)^{\mathrm{ev}} \quad \text { and } \quad B: \mathbb{R}_{>0} \rightarrow(V \otimes V)^{\text {odd }}
$$

are smooth maps. It is easy to check that the second relation in (3) of Theorem 6.42 is equivalent to the matrix decomposition of $\rho$ as in the statement of our corollary.

6.8. Real Euclidian field theories. We recall from Section 6.3 the notion of real structures on cs-manifolds and Euclidean cs-manifolds, see Example 6.20. For

$$
G=\operatorname{Iso}\left(\mathbb{E}^{1 \mid 1}\right)=\mathbb{R}_{\mathrm{cs}}^{1 \mid 1} \rtimes \mathbb{Z} / 4
$$


the real structure on the 4 th roots of unity $\mathbb{Z} / 4$ is obtained by restriction from the usual real structure on $\mathbb{C}$. In particular, $r_{G}(\mathrm{pg})=\mathrm{pg}^{-1}$ which implies the following effect on the generators of the bordism category 1|1-PEB from Theorem 6.42:

$$
\overline{\mathrm{sp}} \cong \mathrm{sp}, \quad \overline{\mathrm{fl}}=\mathrm{fl}, \quad \bar{L}_{0}=L_{0} \circ(\mathrm{fl} \amalg \mathrm{id}) \quad \text { and } \quad \bar{I}_{t, \theta}=I_{t, \theta}
$$

Here we used Lemma 6.19 to get these complex conjugate Euclidean cs-manifolds. This actually induces an involution of complex conjugation on the whole Euclidean cs-bordism category 1|1-PEB $\rightarrow$ csM, compatible with the usual one on csM. Similarly, complex conjugation of complex vector bundles induces such an involution on the fibred category SFr $\rightarrow$ csM.

Definition 6.47. Consider the resulting conjugation action on the groupoid

$$
\operatorname{Fun}_{\mathrm{csM}}^{\otimes, s s}(1 \mid 1-\mathrm{PEB}, \mathrm{SFr}) \text {, }
$$

written as $E \mapsto \bar{E}$. Then the groupoid of real Euclidean field theories is defined to be the homotopy fixed point groupoid of this $\mathbb{Z} / 2$-action and is denoted by

$$
\operatorname{Fun}_{\mathrm{cs} M}^{\otimes, s s}(1 \mid 1-\mathrm{PEB}, \mathrm{SFr})_{\mathbb{R}}
$$

The objects are pairs $\left(E, r_{E}\right)$ where $E \in \mathrm{Fun}_{\mathrm{csM}}^{\otimes, s s}(1 \mid 1-\mathrm{PEB}, \mathrm{SFr})$ and $r_{E}: E \rightarrow \bar{E}$ is a natural isomorphism such that $\bar{r}_{E} \circ r_{E}=\mathrm{id}_{E}$. Morphisms are natural isomorphisms of EFTs that are compatible with the real structures $r_{E}$.

Using Corollary 6.46 and the above relations, we can spell out the data involved in such a real EFT. Because the super-intervals $I_{t, \theta}$ are real (also know as invariant under complex conjugation), the discussion simplifies in the case where the pairing $\mu_{0}=E\left(L_{0}\right)$ is nondegenerate on $V:=E(\mathrm{sp})$, in the sense that the induced map $V \otimes V \rightarrow \operatorname{End}(V)$ is injective. We refer to the image of this map simply as the traceclass operators. In this nondegenerate case one does not lose any information when going from $R_{t, \theta}$ to $I_{t, \theta}=R_{t, \theta} \circ_{\mathrm{sp}} L_{0}$. We simply call such EFTs nondegenerate, adding a superscript nd into the notation.

Theorem 6.48. The groupoid $\mathrm{Fun}_{\mathrm{cs}}^{\otimes, \mathrm{ss}}(1 \mid 1-\mathrm{PEB}, \mathrm{SFr})_{\mathbb{R}}^{\mathrm{nd}}$ of nondegenerate real $1 \mid 1$-dimensional EFTs is equivalent to the groupoid of triples $(W, \mu, \varrho)$ where $W$ is a graded real Fréchet space with an even pairing $\mu: W \otimes W \rightarrow \mathbb{R}$ that is symmetric in the naive sense:

$$
\mu(v, w)=\mu(w, v) \quad \text { for all } v, w \in W .
$$

Moreover, $\varrho: \mathbb{R}_{>0}^{1 \mid 1} \rightarrow \operatorname{End}(W)$ is an even super-semigroup of trace-class operators that is self-adjoint with respect to $\mu$ :

$$
\mu\left(\varrho_{t, \theta} v, w\right)=\mu\left(v, \varrho_{t, \theta} w\right) \quad \text { for all } v, w \in W .
$$

Morphisms in this groupoid are even isomorphisms of Fréchet spaces, compatible with the extra structure given by $\mu, \varrho$.

Proof. We start with a real EFT $\left(E, r_{E}\right)$ and first collect the data given by $E$ as in Corollary 6.46: These are $V=E(\mathrm{sp})$, a complex Fréchet space graded by $\alpha=E(\mathrm{fl})$ with pairing $\mu_{0}=E\left(L_{0}\right)$ and semigroup $\rho=E(R)$. The real structure $r_{E}$ induces a real structure $r_{V}$ on $V$ that is compatible with the grading $\alpha$ because $\overline{\mathrm{fl}}=\mathrm{fl}$. Thus the fixed point set $W$ of $r_{V}$ is a graded real Fréchet space.

The relation $\bar{L}_{0}=L_{0} \circ($ fl $\amalg$ id $)$ implies that

$$
\overline{\mu_{0}\left(r_{V}(v), r_{V}(w)\right)}=\mu_{0}(\alpha(v), w) \quad \text { for all } \quad v, w \in V .
$$


This means that $\mu_{0}$ is real on $W^{\text {ev }}$ but purely imaginary on $W^{\text {odd }}$. That's why we can't use $\mu_{0}$ as the required pairing. However, there is a simple fix to this problem: Define $\mu$ by setting it equal to $\mu_{0}$ on $V^{\text {ev }}$ and to $i \cdot \mu_{0}$ on $V^{\text {odd }}$. Then $\mu$ is a real pairing in the sense that it takes real values on $W$. Also recall that $\mu_{0}$, and by definition $\mu$, are even, i.e., they vanish on vectors of distinct parity.

This factor of $i$ does not only give a real pairing but it also cancels the annoying factor of $i$ in relation (3) of Theorem 6.42: Let $\varrho_{t, \theta}:=E\left(I_{t, \theta}\right): V \rightarrow V$, an even super-semigroup of trace-class operators. Then this relation (3) implies that

$$
\mu_{0}\left(\varrho_{t, \theta} v, w\right)=\mu_{0}\left(v, \varrho_{t, i \theta} w\right) \quad \text { for all } \quad v, w \in V .
$$

We can write $\varrho$ in the form $A+\theta B$ as in the proof of Corollary 6.46. Here

$$
A: \mathbb{R}_{>0} \rightarrow \operatorname{End}(V)^{\text {ev }} \text { and } B: \mathbb{R}_{>0} \rightarrow \operatorname{End}(V)^{\text {odd }}
$$

are smooth maps. Then we check the self-adjointness with respect to $\mu$ instead of $\mu_{0}$, using the fact that both these pairings are even. If $v, w \in V^{\mathrm{ev}}$ or $v, w \in V^{\text {odd }}$ then

$$
\mu\left(\varrho_{t, \theta} v, w\right)=\mu\left(A_{t} v, w\right)=\mu_{0}\left(A_{t} v, w\right)=\mu_{0}\left(v, A_{t} w\right)=\mu\left(v, \varrho_{t, \theta} w\right)
$$

In the case where $v \in V^{\mathrm{ev}}, w \in V^{\text {odd }}$ (or vice versa), we have

$$
\mu\left(\varrho_{t, \theta} v, w\right)=\mu\left(\theta B_{t} v, w\right)=i \mu_{0}\left(\theta B_{t} v, w\right)=\mu_{0}\left(v, \theta B_{t} w\right)=\mu\left(v, \theta B_{t} w\right)=\mu\left(v, \varrho_{t, \theta} w\right)
$$

implying the self-adjointness of $\varrho_{t, \theta}$ with respect to $\mu$. Since $I_{t, \theta}$ is real, the same holds for $\varrho_{t, \theta}$ and we have proven that the triple $(W, \mu, \varrho)$ satisfies all the conditions stated in our corollary.

Conversely, given such a triple, we need to reconstruct the real EFT. This is completely straightforward, except that we have to use the nondegeneracy of $\mu$ (or equivalently, of $\mu_{0}$ ) to reconstruct the super-semigroup $\rho: \mathbb{R}_{>0}^{1 \mid 1} \rightarrow V \otimes V$ from the semigroup $\varrho$. Then the conditions line up exactly.

The last step we need is to nail down the Fréchet space by the following positivity condition that's the super analogue of Definition 6.26. It implies the nondegeneracy required above and we use the notation from the previous theorem.

Definition 6.49. A 1|1-dimensional real Euclidean field theory

$$
E \in \operatorname{Fun}_{\mathrm{csM}}^{\otimes, s s}(1 \mid 1-\mathrm{PEB}, \mathrm{Fr})_{\mathbb{R}}
$$

is nonnegative if $\mu(v, w) \geq 0$ for all $v, w \in W$. We call $E$ positive if $W$ is in addition separable and its even and odd parts are both infinite-dimensional and $\mu$ gives an (algebraic) isomorphism from $W$ to its continuous dual space $W^{\prime}$. The full subgroupoid of $\operatorname{Fun}_{\mathrm{cs} M}^{\otimes, s s}(1 \mid 1-\mathrm{PEB}, \mathrm{Fr})_{\mathbb{R}}$ given by positive field theories is denoted by 1|1-PEFT, the groupoid of positive Euclidean field theories of dimension 1|1.

In the positive case, Lemma 6.3 implies that $W$ is a graded real Hilbert space with even inner product $\mu$. Therefore, $W \otimes W$ is the space of trace-class operators and we obtain the final computation already used as Corollary 6.37 from the fact that any two such Hilbert spaces are isometric. 


\section{Quillen categories and their classifying spaces}

In this section we shall first survey some constructions going back to Quillen and then relate certain $C_{n}$-linear generalizations to our configuration spaces $\operatorname{Conf}_{n}^{\text {fin }}$. Recall from [9] that for any symmetric monoidal category $(C, \oplus)$, Quillen defined the $S^{-1} S$-construction of C, usually denoted by $\mathrm{C}^{-1} \mathrm{C}$, as follows: Objects are pairs $\left(C^{+}, C^{-}\right)$of objects of $\mathrm{C}$ and a morphism from $\left(C^{+}, C^{-}\right)$to $\left(D^{+}, D^{-}\right)$is a triple

$$
\left(A, \alpha^{+}, \alpha^{-}\right) \text {where } A \in \mathrm{C} \text { and } \alpha^{ \pm}: C^{ \pm} \oplus A \rightarrow D^{ \pm}
$$

modulo the relation that two triples $\left(A, \alpha^{+}, \alpha^{-}\right)$and $\left(B, \beta^{+}, \beta^{-}\right)$represent the same morphism if there is an isomorphism $\gamma: A \rightarrow B$ such that $\alpha^{ \pm}=\beta^{ \pm} \circ(\mathrm{id} \oplus \gamma)$. Quillen showed that if $\mathrm{C}$ is a groupoid and adding objects $A \in \mathrm{C}$ defines faithful endofunctors of $C$ then the classifying space of $C^{-1} C$ is the topological group completion of the classifying space of $\mathrm{C}$ (in the world of homotopy commutative and associative H-spaces).

For example, let $C$ be the groupoid of finite-dimensional real inner product spaces (and isometries), with orthogonal sum as monoidal structure. The existence of orthogonal complements simplifies the above definitions and it is easy to see that one obtains the following description of the topological category $D:=C^{-1} \mathrm{C}$ :

- objects of $D$ are graded inner product spaces and

- the morphism spaces $\mathrm{D}\left(W_{1}, W_{2}\right)$ consist of pairs $(f, R)$ where $f: W_{1} \hookrightarrow$ $W_{2}$ is an isometric embedding and $R$ is an odd orthogonal involution on the cokernel $W_{2}-f\left(W_{1}\right)$.

Here and in the following we use the notation $W_{2}-W_{1}$ for the orthogonal complement of $W_{1}$ in $W_{2}$. In this case, a topological version of Quillen's theorem implies that the classifying space

$$
\left|N_{\bullet}(\mathrm{D})\right| \simeq B O \times \mathbb{Z}
$$

because the right-hand side is the group completion of $\left|N_{\bullet}(\mathrm{C})\right| \simeq \coprod_{n} B O(n)$.

To relate these categories to our configurations, let's fix a separable real Hilbert space $H$ of infinite dimension and denote by $\mathrm{C}^{\delta}(H)$ the same topological groupoid as above, except that the objects are required to be subspaces of $H$. Moreover, let $\mathrm{C}(H)$ be the internal groupoid in Top that has the same objects and morphisms as $\mathrm{C}^{\delta}(H)$ but where the the topology on the objects isn't discrete but has the usual topology of the Grassmannian of $H$. It is well known that the two continuous functors

$$
\mathrm{C} \leftarrow \mathrm{C}^{\delta}(H) \rightarrow \mathrm{C}(H)
$$

induce homotopy equivalences on classifying spaces. Note that the two new categories are not monoidal since the direct sum is only partially defined: One needs that two given subspaces of $H$ are orthogonal before one can take their sum (in a way that agrees with the direct sum in C). As a consequence, we cannot directly apply Quillen's $S^{-1} S$-construction (even though there is a version for partially defined symmetric monoidal structures). However, there are obvious ambient analogues of the category D above: Consider $\mathcal{H}=H \oplus H$ as a graded Hilbert space and denote by $\mathrm{D}^{\delta}(\mathcal{H})$, respectively $\mathrm{D}(H)$, the categories in Top having as objects graded subspaces of $\mathcal{H}$ (with the discrete, respectively natural, topology) and with morphism spaces just like for D. Again there are two continuous functors

$$
\mathrm{D} \leftarrow \mathrm{D}^{\delta}(\mathcal{H}) \rightarrow \mathrm{D}(\mathcal{H})
$$


and one can show that they give homotopy equivalences on classifying spaces. In the ambient setting, there is one more simplification one can make. Namely, let $\mathrm{D}_{0}$ be the subcategory of $\mathrm{D}(\mathcal{H})$ with the same space of objects (graded subspaces of $\mathcal{H})$ but with morphisms restricted to those pairs $(f, R)$ where $f$ is an inclusion of subspaces. In particular, there can only be a morphism from $W_{1}$ to $W_{2}$ in $\mathrm{D}_{0}$ if $W_{1} \subseteq W_{2}$. Theorem 7.1 below says that the classifying space of this category in Top has the homotopy type of Conf $f_{0}^{\text {fin }}$. Using the contractibility of $O(H)$ one can show that the continuous functor

$$
\mathrm{D}_{0} \rightarrow \mathrm{D}(\mathcal{H})
$$

also induces a homotopy equivalence on classifying spaces, hence providing an alternative way to see that $\operatorname{Conf}_{0}^{\text {fin }}$ and $B O \times \mathbb{Z}$ have the same homotopy type.

For any graded algebra $A$, there are $A$-linear analogues of the above category $\mathrm{D}$. These are not directly related to the Quillen construction, at least not to our eyes. In the following, we shall make the ambient versions of these categories precise for real Clifford algebras $A=C_{n}$.

Fix a $\mathbb{Z} / 2$-graded real $C_{n}$-module $\mathcal{H}_{n}$ as in the introduction. Using graded $C_{n}$-submodules of $\mathcal{H}_{n}$ as objects, one can define categories $\mathrm{D}^{\delta}\left(\mathcal{H}_{n}\right), \mathrm{D}\left(\mathcal{H}_{n}\right)$ and $\mathrm{D}_{n}$ in Top as follows: The morphism spaces consist of pairs $(f, R)$ as above such that both $f$ and $R$ are $C_{n}$-linear (and $f$ is restricted to inclusions for $\mathrm{D}_{n}$ ). As before one can show that the two continuous functors

$$
\mathrm{D}_{n} \rightarrow \mathrm{D}\left(\mathcal{H}_{n}\right) \leftarrow \mathrm{D}^{\delta}\left(\mathcal{H}_{n}\right)
$$

give homotopy equivalences on classifying spaces; the end of Remark 7.3 contains a very good reference. We shall be mostly concerned with $\mathrm{D}_{n}$ since its classifying space is homeomorphic to a configuration space. So let's make that case completely precise: There is no morphism from $W_{1}$ to $W_{2}$ in $\mathrm{D}_{n}$ unless $W_{1} \subseteq W_{2}$ and in this case

$$
\operatorname{Mor}_{\mathrm{D}_{n}}\left(W_{1}, W_{2}\right):=\left\{R \in O_{C_{n}}\left(W_{2}-W_{1}\right) \mid R^{\alpha}=-R, R^{2}=1\right\} .
$$

The operators $R$ are $C_{n}$-linear, orthogonal, odd involutions on this complement. The \pm 1 -eigenspaces $V_{ \pm}$of $R$ provide an orthogonal decomposition

$$
W_{2}-W_{1}=V_{+} \perp V_{-} \quad \text { with } V_{-}=\alpha\left(V_{+}\right) .
$$

In fact, mapping an operator $R$ as above to its +1 -Eigenspace gives a bijection with the set of subspaces $V_{+} \subset W_{2}-W_{1}$ satisfying the above property. As in Section 4 we think of subspaces of $\mathcal{H}_{n}$ as orthogonal projection operators, hence identifying the set of objects $\mathrm{Obj}_{\mathrm{D}_{n}}$ of $\mathrm{D}_{n}$ with a subspace of $B\left(\mathcal{H}_{n}\right)$. In order to topologize the set of morphisms Mor $\mathrm{D}_{n}$ of $\mathrm{D}_{n}$ we identify it with the set of triples $\left(W_{1}, W_{2}, A\right)$, where $W_{1}, W_{2} \in \mathrm{Obj}_{\mathrm{D}_{n}}$ and $A \in B\left(\mathcal{H}_{n}\right)$ such that $W_{2}-W_{1}$ is an invariant subspace for $A$, the kernel of $A$ is $\left(W_{2}-W_{1}\right)^{\perp}$ and $A$ defines an odd, orthogonal involution on $W_{2}-W_{1}$. We make Mor $_{\mathrm{D}_{n}}$ into a topological space, in fact, a metric space, by considering it as a subspace of the product of three copies of $B\left(\mathcal{H}_{n}\right)$.

The nerve $N_{\bullet}\left(D_{n}\right)$ is a simplicial space, whose geometric realization

$$
\mathcal{Q}_{n}:=\left|N_{\bullet}\left(\mathrm{D}_{n}\right)\right|
$$

is the classifying space of $\mathrm{D}_{n}$ and it is directly related to configuration spaces:

Theorem 7.1. There is a bijective continuous map $\widetilde{G}_{n}: \mathcal{Q}_{n} \stackrel{\simeq}{\rightarrow} \operatorname{Conf}_{n}^{\text {fin }}$ that is also a homotopy equivalence. 
Proof. It suffices to consider the case $n=0$. In this case, we suppress the index $n$ altogether. In order to get the claim for general $n$, apply the $n=0$ case to $\mathcal{H}_{n}$ (merely considered as a $\mathbb{Z}_{2}$-graded real Hilbert space) to obtain a bijective continuous map $\widetilde{G}: B \mathrm{D} \approx \mathrm{Conf}^{\text {fin }}$. It is clear from the definition of $\widetilde{G}$ (see below) that the classifiying space $B \mathrm{D}_{n} \subset B \mathrm{D}$ of the subcategory $\mathrm{D}_{n}$ of $\mathrm{D}$ corresponds precisely to $\operatorname{Conf}_{n}^{\text {fin }} \subset \operatorname{Conf}^{\text {fin }}$ under $\widetilde{G}$. Since the homotopy used to prove that $\widetilde{G}$ is a homotopy equivalence preserves the subspace $\operatorname{Conf}_{n}^{\text {fin }}$, the claim follows for general $n$.

Recall that the $k$-simplices $x \in N_{k} \mathrm{D}$ of the nerve of our internal space category $\mathrm{D}$ are chains of $\mathbb{Z} / 2$-graded finite-dimensional subspaces

$$
W_{0} \subseteq W_{1} \subseteq \cdots \subseteq W_{k} \subset \mathcal{H}
$$

together with odd, orthogonal involutions $R_{i}$ on $W_{i}-W_{i-1}$ for $i=1, \ldots, k$. We abbreviate this to $x=\left(W_{i}, R_{i}\right)$. The classifying space $B \mathrm{D}$ is the quotient space

$$
\left(\coprod_{k \geq 0} N_{k} \mathrm{D} \times \Delta^{k}\right) /\left(\beta^{*}(x), t\right) \sim\left(x, \beta_{*}(t)\right) \quad \forall \beta:[m] \rightarrow[n]
$$

In our context, it is convenient to replace the usual standard simplex by

$$
\Delta^{k}:=\left\{t=\left(t_{1}, \ldots, t_{k}\right) \in \overline{\mathbb{R}}^{k} \mid 0 \leq t_{1} \leq \cdots \leq t_{k} \leq \infty\right\} .
$$

The face map $d_{i}:[k-1] \rightarrow[k]$ induces the map $\left(d_{i}\right)_{*}: \Delta^{k-1} \hookrightarrow \Delta^{k}$, given by repeating $t_{i}$, for $i=1, \ldots, k$. Moreover, $\left(d_{0}\right)_{*}$ adds a first coordinate equal to 0 and $\left(d_{k}\right)_{*}$ adds a last coordinate equal to $\infty$. For $i=0, \ldots, k-1$, the degeneracy maps $s_{i}:[k] \rightarrow[k-1]$ induce $\left(s_{i}\right)_{*}: \Delta^{k} \rightarrow \Delta^{k-1}$, given by skipping $t_{i+1}$.

Now, every $(x, t) \in N_{k} \mathcal{C} \times \Delta^{k}$ defines a configuration $G(x, t) \in$ Conf $^{f i n}$ as follows. The label $G(x, t)_{0}$ at zero is $W_{i}$, where $i$ is the largest index with $t_{i}=0$. For $0<\lambda<\infty, G(x, t)_{ \pm \lambda}$ is the sum of the \pm 1 -eigenspaces of all operators $R_{i}$ with indices $i$ with $t_{i}=\lambda$. The label $G(x, t)_{\infty}$ is the orthogonal complement of all the other $G(x, t)_{\lambda}$ 's. We claim that the map

$$
\widetilde{G}: B \mathrm{D} \rightarrow \mathrm{Conf}^{\mathrm{fin}}, \quad \widetilde{G}[x, t]:=G(x, t)
$$

is well defined. We have to check that for all $(x, t)$ and face and degeneracy maps $\beta$ we have $G\left(\beta^{*}(x), t\right)=G\left(x, \beta_{*}(t)\right)$.

We start with the face maps. In these cases we write $t=\left(t_{1}, \ldots, t_{k-1}\right)$. If $\beta=d_{0}:[k-1] \rightarrow[k]$ then $\beta^{*}(x)$ is the chain of subspaces where $W_{0}$ and $R_{1}$ have been removed (and the indices of the other $W_{i}$ and $R_{i}$ are shifted to the left). Since $\beta_{*}(t)=\left(0, t_{1}, \ldots, t_{k-1}\right)$ it is clear from the definition of $G$ that the labels of $G\left(\beta^{*}(x), t\right)$ and $G\left(x, \beta_{*}(t)\right)$ coincide.

For $i=1, \ldots, k-1$ and $\beta=d_{i}$, the chain $\beta^{*}(x)$ is obtained by composing the morphisms $R_{i}$ and $R_{i+1}$. This means that on $W_{i+1}-W_{i-1}$ we get an orthogonal sum of these two operators. Since the \pm 1 -eigenspaces of this orthogonal sum equals the direct sum of the \pm 1 -eigenspaces of $R_{i}$ and $R_{i+1}$ and since $\beta_{*}(t)$ just repeats $t_{i}$, it is clear that $G\left(\beta^{*}(x), t\right)_{t_{i}}=G\left(x, \beta_{*}(t)\right)_{t_{i}}$. All the other labels are clearly unchanged so that $G\left(\beta^{*}(x), t\right)=G\left(x, \beta_{*}(t)\right)$.

If $\beta=d_{k}:[k-1] \rightarrow[k]$ then $\beta^{*}(x)$ is the chain of subspaces where $W_{k}$ and $R_{k}$ have been removed. However, since $\beta_{*}(t)=\left(t_{1}, \ldots, t_{k-1}, \infty\right)$, we have $G\left(\beta^{*}(x), t\right)_{\lambda}=G\left(x, \beta_{*}(t)\right)_{\lambda}$ for all $\lambda \in \mathbb{R}$ and so $G\left(\beta^{*}(x), t\right)=G\left(x, \beta_{*}(t)\right)$.

For a degeneracy map $\beta=s_{i}:[k] \rightarrow[k-1]$, where $i=0, \ldots, k-1$, the argument is even easier. Then for a $(k-1)$-simplex $x$, we get a chain $\beta^{*}(x)$ of 
length $k$ by inserting the identity at the $i$ th subspace. This operation does not alter the operators $R_{j}$ (the identity corresponds to $R=0$ on a 0 -space), it only shifts the indices $>i$ to the right. Similarly, $\beta_{*}\left(t_{1}, \ldots, t_{k}\right)=\left(t_{1}, \ldots, \hat{t}_{i}, \ldots, t_{k}\right)$, so that again a shifting of indices $>i$ to the right occurs and $G\left(\beta^{*}(x), t\right)=G\left(x, \beta_{*}(t)\right)$ follows.

Hence the map $\widetilde{G}$ is well defined. It is bijective, since it has an inverse Conf $^{\text {fin }} \rightarrow B D$ defined by mapping a configuration $\left\{V_{\lambda}\right\}$ with exactly $k$ nontrivial labels $V_{\lambda_{1}}, \ldots, V_{\lambda_{k}}$ with $0<\lambda_{i}<\infty$ to the equivalence class $[x, t]$, where $x$ is defined by the chain $W_{0} \subseteq \cdots \subseteq W_{k}$, where $W_{0}=V_{0}$ and

$$
W_{i}:=W_{i-1} \oplus V_{\lambda_{i}} \oplus V_{-\lambda_{i}}
$$

for $i>0$ and the operator $R_{i}$ on $V_{\lambda_{i}} \oplus V_{-\lambda_{i}}$ is defined to be the one with the \pm 1 -eigenspaces $V_{ \pm \lambda_{i}}$. It is clear that this defines an inverse for $\widetilde{G}$.

It is easy to see that $\widetilde{G}$ is continuous: the description of the neighborhood basis

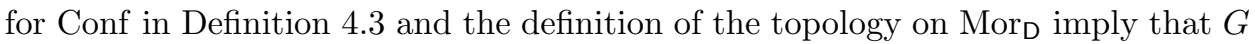
is continuous. Hence the same is true for $\widetilde{G}$.

It remains to show that $\widetilde{G}$ is a homotopy equivalence. Let $\operatorname{Conf}^{(k)} \subset \operatorname{Conf}^{\text {fin }}$ be the subspace defined in Remark 4.7 and denote by $B \mathrm{D}^{(k)}$ the image of $N_{k} \mathrm{e} \times \Delta^{k}$ in BD. According to Lemma 7.2 below, $\widetilde{G}$ restricts to a homeomorphism

$$
\widetilde{G}^{(k)}: B \mathrm{D}^{(k)} \rightarrow \mathrm{Conf}^{(k)}
$$

for all $k \geq 0$. This together with the fact that id: $\operatorname{colim}_{k \rightarrow \infty} \operatorname{Conf}^{(k)} \rightarrow \operatorname{Conf}^{\text {fin }}$ (see Remark 4.7) implies that $\widetilde{G}$ is a homotopy equivalence. This completes the proof of Theorem 7.1.

Lemma 7.2. For all $k \geq 0$, the map

$$
\widetilde{G}^{(k)}: B \mathrm{D}^{(k)} \rightarrow \operatorname{Conf}^{(k)}
$$

is a homeomorphism.

Proof. Since $\widetilde{G}$ is continuous, so is $\widetilde{G}^{(k)}$. The proof that $\widetilde{G}^{(k)}$ is open is based on the following fact.

Fact. There is a function $\varepsilon_{k}:\left(0, \frac{1}{2}\right) \rightarrow \mathbb{R}_{>0}$ satisfying $\varepsilon_{k}(\delta) \rightarrow 0$ as $\delta \rightarrow 0$ with the following property. If $l \leq k$ and $V, W_{1}, W_{2}, \ldots, W_{l}$, are finite-dimensional subspaces of a Hilbert space $H$ such that $\left\|P_{V}-P_{\oplus_{i=1}^{l} W_{i}}\right\|<\delta<\frac{1}{2}$ and the $W_{i}$ 's are mutually orthogonal, then there exists a splitting of $V$ into mutually orthogonal subspaces $V_{1}, \ldots, V_{k}$ such that $\left\|P_{V_{i}}-P_{W_{i}}\right\|<\varepsilon_{k}(\delta)$ for all $i$.

The idea of the proof is simple. The subspaces $\tilde{W}_{i}:=P_{V}\left(W_{i}\right) \subset V$ give a decomposition of $V$ and the $\tilde{W}_{i}$ are almost orthogonal. Then use Gram-Schimdt to orthogonalize the $\tilde{W}_{i}$. Standard estimates plus induction on $k$ yield the desired function $\varepsilon_{k}$ (e.g., $\varepsilon_{k}(\delta)=3^{k-1} \delta^{2^{-(k-1)}}$ works).

Now, given a neighborhood $N$ of $[x, t] \in B \mathrm{D}^{(k)}$ we have to find $K>0$ and $\delta>0$ such that $V_{K, \delta, \delta} \cap \operatorname{Conf}^{(k)} \subset \widetilde{G}^{(k)}(N)$, where $V:=\widetilde{G}^{(k)}[x, t]$ and $V_{K, \delta, \delta}$ is the neighborhood of $V$ described in Definition 4.3. We may choose $K$ to be any number such that $(-K-1, K+1)$ contains all nontrivial labels $\lambda \in \mathbb{R}$ of $V$. In order to find $\delta$, observe that the preimage of $N$ in $\coprod_{i=0}^{k} N_{i} \mathrm{D} \times \Delta^{i}$ contains a full $\varepsilon$-neighborhood of the equivalence class $[x, t]$ (recall that $N_{i} \mathrm{D} \times \Delta^{i}$ is a metric space for all $i$ ) for 
some $\varepsilon>0$. The existence of $\varepsilon$ follows from the Lebesgue covering lemma and the fact that $[x, t]$ is compact.

Now choose $\delta>0$ such that $\varepsilon_{k}(\delta)<\varepsilon$. In order to show that $V_{K, \delta, \delta} \cap \operatorname{Conf}^{(k)} \subset$ $\widetilde{G}^{(k)}(N)$, consider any $W \in V_{K, \delta, \delta} \cap \operatorname{Conf}^{(k)}$. Let $j$ be minimal with $W \in \operatorname{Conf}^{(j)}$. Using the Fact it is easy to see that there is $(y, s) \in[x, t] \cap N_{j} \mathrm{D} \times \Delta^{j}$ that lies within $\varepsilon$-range of the unique preimage of $W$ in $N_{j} \mathrm{D} \times \Delta^{j}$ under $G$. In other words, $(y, s)$ lies within $\varepsilon$-distance from $[x, t]$. Hence the preimage of $W$ under $\widetilde{G}^{(k)}$ lies in $N$, as desired. This completes the proof that $\widetilde{G}^{(k)}$ is open.

Remark 7.3. In his thesis [5], Pokman Cheung considers Conf $_{n}$ as a discrete set of objects and introduces a space of morphisms which gives a topological category that we shall denote by $\mathrm{C}^{\delta}\left(\mathcal{H}_{n}\right)$. He refers to this as the "category of 1-dimensional super Euclidean field theories," even though the morphisms are not defined in terms of field theories but rather in terms of configurations in $\mathcal{H}_{n}$, i.e., points in $\operatorname{Conf}_{n}$. This is reflected by the fact that in the 2-dimensional case, Cheung can only define an analogous category for field theories based solely on annuli where one again can express the data in terms of configurations of Eigenspaces of an infinitesimal generator.

The space of morphisms in $\mathrm{C}^{\delta}\left(\mathcal{H}_{n}\right)$ is closely related to the path-space of Conf ${ }_{n}$. Via the Moore method, the space of paths actually gives a more direct way to get a topological category with classifying space $\operatorname{Conf}_{n}$. However, $\mathrm{C}^{\delta}\left(\mathcal{H}_{n}\right)$ has the advantage that it connects very well to the Quillen type category $\mathrm{D}^{\delta}\left(\mathcal{H}_{n}\right)$ discussed at the beginning of this section.

There are two canonical maps on objects from $\mathrm{C}^{\delta}\left(\mathcal{H}_{n}\right)$ to $\mathrm{D}^{\delta}\left(\mathcal{H}_{n}\right)$ and back: One map takes a configuration $\left\{V_{\lambda}\right\} \in \operatorname{Conf}_{n}$ and maps it to $V_{0} \subset \mathcal{H}_{n}$, the index or $\mathbb{Z} / 2$-graded kernel of the odd infinitesimal generator corresponding to the configuration. This map is not continuous in the natural topologies and hence the use of discrete topologies is essential. The other canonical map (which is continuous in the natural topologies) starts with a finite-dimensional $C_{n}$-submodule $W \subset \mathcal{H}_{n}$ and associates to it the configuration with $V_{0}:=W$ and no other nontrivial eigenspaces.

The new idea in [5] is to define the morphisms in $C^{\delta}\left(\mathcal{H}_{n}\right)$ in a way such that these two maps extend to continuous functors between topological categories. In fact, a main result [5, Proposition 1.4.1] says that the classifying spaces of $\mathrm{C}^{\delta}\left(\mathcal{H}_{n}\right)$ and $\mathrm{D}^{\delta}\left(\mathcal{H}_{n}\right)$ are homotopy equivalent via these two inverse functors. Moreover, it is shown that the latter classifying space is homotopy equivalent to the AtiyahSinger space $\mathcal{F}_{n}$ studied in the next section. The first steps of the argument [5, Lemmas 1.4.3, 1.4.4] actually show that the classifying spaces of $\mathrm{D}^{\delta}\left(\mathcal{H}_{n}\right)$ and $\mathrm{D}_{n}$ are homotopy equivalent:

$$
\left|N_{\bullet} \mathrm{D}\left(\mathcal{H}_{n}\right)\right| \simeq\left|N_{\bullet} \mathrm{D}_{n}\right|=Q_{n}
$$

The rest of Cheung's argument is thus an alternative to our proof, in this and the next section, where we exhibit canonical homotopy equivalences $Q_{n} \simeq \operatorname{Conf}_{n} \simeq \mathcal{F}_{n}$.

\section{Spaces of Fredholm operators}

In this chapter we relate the spaces $\operatorname{Conf}_{n}$ to the spaces of skew-adjoint Fredholm operators considered by Atiyah and Singer in [2]. See the introduction for a quick survey of this section. 
8.1. Fredholm operators. Recall that a Fredholm operator is a bounded operator whose kernel and cokernel are finite-dimensional. Let Fred $(H) \subset B(H)$ be the subspace of Fredholm operators on the infinite-dimensional separable real Hilbert space $H$. Denote by $C(H):=B(H) / K(H)$ the $C^{*}$-algebra of bounded operators modulo compact operators (also known as Calkin algebra) and by $\pi: B(H) \rightarrow$ $C(H)$ the projection. Then $\operatorname{Fred}(H)$ is the preimage of the group of units in $C(H)$ under $\pi$, i.e., we have

$$
T \in B(H) \text { is Fredholm } \Longleftrightarrow \pi(T) \in C(H) \text { is invertible. }
$$

We will need the following facts about the spectrum $\sigma(T)$ of a self-adjoint bounded operator $T$. Let $\sigma_{\text {ess }}(T):=\sigma(\pi(T))$ be the essential spectrum of $T$, i.e., the spectrum of $\pi(T)$ in $C(H)$. Then there is a decomposition

$$
\sigma(T)=\sigma_{\text {ess }}(T) \amalg \sigma_{\text {discrete }}(T),
$$

where $\sigma_{\text {discrete }}(T)$ consists precisely of the isolated points in $\sigma(T)$ such that the corresponding eigenspace has finite dimension. From the definition of the essential spectrum it is clear that for a Fredholm operator $T$

$$
\sigma_{\text {ess }}(T) \cap(-\varepsilon(T), \varepsilon(T))=\varnothing \text { for } \varepsilon(T):=\left\|\pi(T)^{-1}\right\|_{C(H)}^{-1},
$$

where $\|\cdot\|_{C(H)}$ is the $C^{*}$-norm on the Calkin algebra. In other words: the essential spectrum of $T$ has a gap of size $\varepsilon(T)$ around 0 . Note that the map $\varepsilon:$ Fred $(H) \rightarrow$ $\mathbb{R}_{>0}$ is continuous.

8.2. $K$-theory and Fredholm operators. The most important invariant of a Fredholm operator $T$ is its index

$$
\operatorname{index}(T):=\operatorname{dim}(\operatorname{ker} T)-\operatorname{dim}(\operatorname{coker} T) .
$$

It turns out that the index is invariant under deformations, i.e., it is a locally constant function on $\operatorname{Fred}(H)$. In fact, it defines an isomorphism

$$
\pi_{0} \operatorname{Fred}(H) \stackrel{\cong}{\longrightarrow} \mathbb{Z}, \quad[T] \mapsto \operatorname{index}(T) .
$$

This is a special case of the well-known result that $\operatorname{Fred}(H)$ is a classifying space for the real $K$-theory functor $K O^{0}$. More explicitly, for compact spaces $X$ there are natural isomorphisms

$$
K O^{0}(X) \cong[X, \operatorname{Fred}(H)] .
$$

This isomorphism is defined as follows. Consider $[f] \in[X, \operatorname{Fred}(H)]$. Changing $f$ by a homotopy one can achieve that the dimensions of the kernel and the cokernel of $f(x)$ are locally constant. This implies that they define vector bundles ker $f$ and coker $f$ over $X$. The image of $[f]$ is defined to be

$$
[\operatorname{ker} f]-[\operatorname{coker} f] \in K O^{0}(X) .
$$

For $X=$ pt this reduces to the above isomorphism

$$
\pi_{0} \operatorname{Fred}(H) \cong[\mathrm{pt}, \operatorname{Fred}(H)] \stackrel{\cong}{\rightrightarrows} K O^{0}(\mathrm{pt}) \cong \mathbb{Z}
$$

Atiyah and Singer showed that the other spaces in the $\Omega$-spectrum representing real $K$-theory can also be realized as spaces of Fredholm operators. 
8.3. The Atiyah - Singer spaces $\mathcal{F}_{\boldsymbol{n}}$. Let $n \geq 1$ and let $H_{n}$ be a real Hilbert space with an action of $C_{n-1}$, just as before. Define

$$
\widetilde{\mathcal{F}}_{n}:=\left\{T_{0} \in \operatorname{Fred}\left(H_{n}\right) \mid T_{0}^{*}=-T_{0} \text { and } T_{0} e_{i}=-e_{i} T_{0} \text { for } i=1 \ldots, n-1\right\} .
$$

Furthermore, let $\mathcal{F}_{n}:=\widetilde{\mathcal{F}}_{n}$ if $n \not \equiv 3(\bmod 4)$. In the case $n \equiv 3(\bmod 4)$ define $\mathcal{F}_{n} \subset \widetilde{\mathcal{F}}_{n}$ to be the subspace of operators $T_{0}$ satisfying the following additional condition (AS): the essential spectrum of the self-adjoint operator $e_{1} \cdots e_{n-1} T_{0}$ contains positive and negative values (we say that $e_{1} \cdots e_{n-1} T_{0}$ is neither essentially positive nor negative). Atiyah and Singer introduce this condition, because it turns out that for $n \equiv 3(\bmod 4)$ the space $\widetilde{\mathcal{F}}_{n}$ has three connected components, two of which are contractible. However, for the relation with $K$-theory only the third component, whose elements are characterized by the above requirement on the essential spectrum of $e_{1} \cdots g e_{n-1} T_{0}$, is interesting. In fact, the main result of [2] is that for all $n \geq 1$ the space $\mathcal{F}_{n}$ represents the functor $K O^{-n}$. We shall re-prove this result in terms of our configuration spaces.

The elements in $\widetilde{\mathcal{F}}_{n}$ can also be interpreted as odd operators on the $\mathbb{Z}_{2}$-graded Hilbert space $\mathcal{H}_{n}=H_{n} \otimes_{C_{n}^{\text {ev }}} C_{n}$. If we define

$$
\widetilde{\mathcal{F}}_{n}^{\mathrm{gr}}:=\left\{T \in \operatorname{Fred}\left(\mathcal{H}_{n}\right) \mid T \text { is odd, } C_{n} \text {-linear, and self-adjoint }\right\}
$$

we can identify $\widetilde{\mathcal{F}}_{n}$ and $\widetilde{\mathcal{F}}_{n}^{\text {gr }}$ using the homeomorphism

$$
\psi_{\otimes e_{n}}: \widetilde{\mathcal{F}}_{n} \underset{\sim}{\rightarrow} \widetilde{\mathcal{F}}_{n}^{\mathrm{gr}}, \quad T_{0} \mapsto T:=T_{0} \otimes e_{n} .
$$

The operator $T$ has the matrix representation

$$
T=\left(\begin{array}{cc}
0 & T_{0}^{*} \\
T_{0} & 0
\end{array}\right)
$$

with respect to the decomposition $\mathcal{H}_{n} \cong H_{n} \oplus H_{n}$. It is important to note that the skew-symmetry of $T_{0}$ is equivalent to the relation $T e_{n}=e_{n} T$.

In the next lemma, we will show that $\widetilde{\mathcal{F}}_{n}^{\text {gr }}$ is homotopy equivalent to a configuration space. Let $\widetilde{\mathbb{R}}:=[-\infty, \infty]$ be the two-point compactification of $\mathbb{R}$ equipped with the involution $s(x):=-x$.

Lemma 8.1. The subspace $\mathcal{A} \subset \widetilde{\mathcal{F}}_{n}^{\mathrm{gr}}$ of all operators $T$ with $\|T\|=1$ and $\varepsilon(T)=1$, see $(*)$ for the definition of $\varepsilon(T)$, is a strong deformation retract of $\widetilde{\mathcal{F}}_{n}^{\mathrm{gr}}$. Furthermore, $\mathcal{A}$ is homeomorphic to the configuration space $\operatorname{Conf}_{C_{n}}\left(\widetilde{\mathbb{R}},\{ \pm \infty\} ; \mathcal{H}_{n}\right)$ as defined in Chapter 4.

Proof. Define a homotopy $H: \widetilde{\mathcal{F}}_{n}^{\mathrm{gr}} \times[0,1] \rightarrow \widetilde{\mathcal{F}}_{n}^{\mathrm{gr}}$ by

$$
(T, t) \mapsto H_{t}(T):=(t+(1-t)\|T\|) \cdot \phi\left(\left(t \varepsilon(T)^{-1}+(1-t)\|T\|^{-1}\right) \cdot T\right) .
$$

Here $\phi: \mathbb{R} \rightarrow[-1,1]$ is defined by $\left.\phi\right|_{[-1,1]}=\mathrm{id},\left.\phi\right|_{[1, \infty)} \equiv 1$ and $\left.\phi\right|_{(\infty,-1]} \equiv-1$, and $\phi(\cdot)$ denotes the functional calculus with $\phi$. The continuity of $H$ follows from the continuity of $\|\cdot\|$ and $\varepsilon$ and from the usual continuity properties of functional calculus, see [24, Theorem VIII.20]. Also, $C_{n}$-linearity and parity of $T$ are preserved under functional calculus. Furthermore,

$$
H_{0}=\mathrm{id}_{\widetilde{\mathcal{F}}_{n}^{\mathrm{gr}}}, \quad H_{t}=\mathrm{id}_{\mathcal{A}} \text { for all } t, \text { and } H_{1}\left(\widetilde{\mathcal{F}}_{n}^{\mathrm{gr}}\right) \subset \mathcal{A} .
$$

Hence $\mathcal{A}$ is a strong deformation retract of $\widetilde{\mathcal{F}}_{n}^{\mathrm{gr}}$. 
Now, for all $T \in \mathcal{A}$ we have $\sigma(T) \subset[-1,1]$ and all $\lambda \in \sigma(T) \cap(-1,1)$ are eigenvalues of finite multiplicity. The spectral theorem for self-adjoint operators implies that the eigenspaces $V(T)_{\lambda}$ of $T$ are pairwise orthogonal and span all of $\mathcal{H}_{n}$. Since $T$ is odd, $V(T)_{-\lambda}=\alpha\left(V(T)_{\lambda}\right)$, where $\alpha$ is the grading involution on $\mathcal{H}_{n}$. We thus obtain a map

$$
\mathcal{A} \rightarrow \operatorname{Conf}_{C_{n}}\left([-1,1],\{ \pm 1\} ; \mathcal{H}_{n}\right), \quad T \mapsto V(T)
$$

by associating to $T$ the configuration $\lambda \mapsto V(T)_{\lambda}$ on $[-1,1]$. Here the involution on $[-1,1]$ is $x \mapsto-x$. It is easy to see that this map is a homeomorphism. Finally, $[-1,1] \stackrel{\approx}{\rightarrow} \widetilde{\mathbb{R}}, x \mapsto x /(1-|x|)$ induces a homeomorphism of configuration spaces $\operatorname{Conf}_{C_{n}}\left([-1,1],\{ \pm 1\} ; \mathcal{H}_{n}\right) \approx \operatorname{Conf}_{C_{n}}\left(\widetilde{\mathbb{R}},\{ \pm \infty\} ; \mathcal{H}_{n}\right)$, thus completing the proof of the second statement in the lemma.

Now we can formulate the relationship between the Atiyah-Singer spaces $\mathcal{F}_{n}$ and our configuration spaces Conf ${ }_{n}$. Consider the map $p: \widetilde{\mathbb{R}} \rightarrow \overline{\mathbb{R}}$ that is the identity on $\mathbb{R}$ and that maps $\pm \infty$ to $\infty$. It induces a continuous map

$$
p_{*}: \operatorname{Conf}_{C_{n}}\left(\widetilde{\mathbb{R}},\{ \pm \infty\} ; \mathcal{H}_{n}\right) \rightarrow \operatorname{Conf}_{C_{n}}\left(\overline{\mathbb{R}},\{\infty\} ; \mathcal{H}_{n}\right)=\operatorname{Conf}_{n} .
$$

Let $H$ be the homotopy equivalence defined as the composition

$$
H: \widetilde{\mathcal{F}}_{n} \underset{\sim}{\rightarrow} \widetilde{\mathcal{F}}_{n}^{\mathrm{gr}} \stackrel{\simeq}{\rightarrow} \operatorname{Conf}_{C_{n}}\left(\widetilde{\mathbb{R}},\{ \pm \infty\} ; \mathcal{H}_{n}\right) .
$$

The main result of this section is:

Theorem 8.2. For all $n \geq 1, p_{*} H$ restricts to a homotopy equivalence

$$
\left.p_{*} H\right|_{\mathcal{F}_{n}}: \mathcal{F}_{n} \stackrel{\simeq}{\rightarrow} \operatorname{Conf}_{n} .
$$

Proof. Since $H$ is a homotopy equivalence, the same is true for $\left.H\right|_{\mathcal{F}_{n}}$. It remains to show that the restriction of $p_{*}$ to the path component $H\left(\mathcal{F}_{n}\right)$ of $\operatorname{Conf}_{C_{n}}\left(\widetilde{\mathbb{R}},\{ \pm \infty\} ; \mathcal{H}_{n}\right)$ is a homotopy equivalence. In order to do this, it will be convenient to work with subspaces consisting of certain 'finite' elements. More precisely, if we define

$$
\operatorname{Conf}_{n}^{\prime}:=H\left(\mathcal{F}_{n}\right) \cap \operatorname{Conf}_{C_{n}}^{\text {fin }}\left(\widetilde{\mathbb{R}},\{ \pm \infty\} ; \mathcal{H}_{n}\right),
$$

then we have a commutative diagram

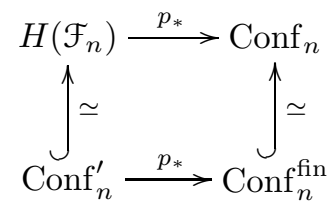

whose vertical arrows are homotopy equivalences (for the right arrow this was done in Proposition 4.6; the same argument works for the arrow on the left). Hence $\left.p_{*}\right|_{H\left(\mathcal{F}_{n}\right)}$ is a weak homotopy equivalence exactly if this is the case for $p_{*}:$ Conf $_{n}^{\prime} \rightarrow$ Conf $_{n}^{\text {fin }}$. This will be proved in Theorem 8.5 below. It follows that $\left.p_{*} H\right|_{\mathcal{F}_{n}}$ is a weak homotopy equivalence. Since $\mathcal{F}_{n}$ and $\operatorname{Conf}_{n}$ both have the homotopy type of a CWcomplex, the map $\left.p_{*} H\right|_{\mathcal{F}_{n}}$ is a homotopy equivalence, cf. [21].

As a first step towards Theorem 8.5, let us give a characterization of the configurations contained the subspace $\operatorname{Conf}_{n}^{\prime}$. Since the map $H$ is surjective, we have $\operatorname{Conf}_{n}^{\prime}=\operatorname{Conf}_{C_{n}}^{\text {fin }}\left(\widetilde{\mathbb{R}},\{ \pm \infty\} ; \mathcal{H}_{n}\right)$ for $n \not \equiv 3(\bmod 4)$ (recall that $\widetilde{\mathcal{F}}_{n}=\mathcal{F}_{n}$ in this case). 
The interesting case is $n \equiv 3(\bmod 4)$. Our task is to understand what the Atiyah-Singer condition (AS) means for the corresponding configurations.

Before we proceed, we need to collect some representation theoretic facts about graded Clifford modules. Recall from [17] that for $n \not \equiv 3(\bmod 4)$ the Clifford algebra $C_{n}$ is simple, whereas it is the product of two simple algebras in the other cases. Therefore, there is a unique irreducible $C_{n}$-module (and a unique graded irreducible $C_{n+1}$-module) for $n \not \equiv 3(\bmod 4)$, otherwise there are exactly two such modules.

Remark 8.3. Let $n \equiv 3(\bmod 4)$ and recall from [17, Chapter I, Proposition 5.9] that in this case the action of the volume element $e:=e_{1} \cdots e_{n} \in C_{n}$ distinguishes the two distinct (ungraded) irreducible $C_{n}$-modules. (Since $e$ is a central orthogonal involution, it acts as \pm id on these modules.)

Now, let $T \in \widetilde{\mathcal{F}}_{n}^{\mathrm{gr}}$. The diagonal entries of the even operator $e T$ are given by $e_{1} \cdots e_{n-1} T_{0}: H_{n} \rightarrow H_{n}$ and therefore the Atiyah-Singer condition (AS) that the operator $e_{1} \cdots e_{n-1} T_{0}: H_{n} \rightarrow H_{n}$ is neither essentially positive nor negative is equivalent to the same condition on $e T: \mathcal{H}_{n} \rightarrow \mathcal{H}_{n}$.

Denote by $\mathcal{A}^{\text {fin }} \subset \mathcal{A}$ the subspace of operators with finite spectrum.

Lemma 8.4. Assume $n \equiv 3(\bmod 4)$. Let $T \in \mathcal{A}^{\text {fin }}$ and denote by $W_{ \pm}$the $( \pm 1)$-eigenspaces of $T$.

(1) $e T$ is essentially positive (respectively negative) if and only if the volume element e has a finite-dimensional (-1)-eigenspace on $W_{+}$(respectively $\left.W_{-}\right)$.

(2) The (AS) condition is equivalent to the $( \pm 1)$-eigenspaces of e, restricted to $W_{+}$, both being infinite-dimensional.

(3) $W_{+}$is a $C_{n}$-module and the (AS) condition is equivalent to $W_{+}$containing both irreducible $C_{n}$-modules infinitely often.

Proof. For part (1) we observe that $e T=T e$ and hence we can find simultanous eigenspace decompositions for these self-adjoint operators. Note that the eigenvalues $( \pm 1)$ are the only possible accumulation points in the spectrum of $T$ and hence such eigenspace decompositions exist. Since $e^{2}=1$, the operator $e$ has spectrum inside $\{ \pm 1\}$.

A vector $v \in \mathcal{H}_{n}$ is in an essentially positive eigenspace of $e T$ if and only if either $v \in W_{+}$and $e(v)=+1$, or $v \in W_{-}$and $e(v)=-1$. Since $T$ is odd, its spectrum is symmetric and, in particular, the grading involution $\alpha$ takes $W_{+}$to $W_{-}$. Furthermore, $\alpha$ anticommutes with $e$ and hence

$$
e_{\mid W_{+}}=e \circ \alpha_{\mid W_{-}}=-\alpha \circ e_{\mid W_{-}}
$$

so that $\alpha$ takes the $(+1)$-eigenspace of $e_{\mid W_{+}}$to the (-1)-eigenspace of $e_{\mid W_{-}}$. In particular, these vector spaces have the same dimension. This finishes the proof of part (1) as well as part (2).

To prove part (3) notice that $T$ is $C_{n}$-linear and therefore $W_{+}$is a $C_{n}$-module (which is not graded since $\alpha$ takes it to $W_{-}$). The claim follows from the well known algebraic fact stated at the beginning of the lemma.

Note that $\operatorname{Conf}_{n}^{\prime}$ is exactly the image of $\mathcal{A}^{\text {fin }} \cap \mathcal{F}_{n}^{\text {gr }}$ under the identification $\mathcal{A} \approx \operatorname{Conf}_{C_{n}}\left(\widetilde{\mathbb{R}},\{ \pm \infty\} ; \mathcal{H}_{n}\right)$ from Lemma 8.1. Moreover, the $( \pm 1)$-eigenspaces $W_{ \pm}$ 
of an operator $T \in \mathcal{A}^{\text {fin }}$ turn into the $( \pm \infty)$-eigenspaces $W_{ \pm \infty}$ of the corresponding configuration $W \in \operatorname{Conf}_{C_{n}}^{\text {fin }}\left(\widetilde{\mathbb{R}},\{ \pm \infty\} ; \mathcal{H}_{n}\right)$.

Hence part $(3)$ of Lemma 8.4 tells us that for $n \equiv 3(\bmod 4)$ the subspace $\operatorname{Conf}_{n}^{\prime} \subset \operatorname{Conf}_{C_{n}}^{\text {fin }}\left(\widetilde{\mathbb{R}},\{ \pm \infty\} ; \mathcal{H}_{n}\right)$ consists precisely of the configurations $\left\{W_{\lambda}\right\}$ such that $W_{ \pm \infty}$ contains both irreducible $C_{n}$-modules infinitely often.

We will now see why this is a very natural condition in terms of our configuration spaces:

Theorem 8.5. The restriction

$$
p:=\left.p_{*}\right|_{\operatorname{Conf}_{n}^{\prime}}: \operatorname{Conf}_{n}^{\prime} \rightarrow \operatorname{Conf}_{n}^{\text {fin }}
$$

is a quasi-fibration with contractible fibers (see Definition 8.8).

Remark 8.6. In the case $n \equiv 3(\bmod 4)$ the space $\operatorname{Conf}_{n}^{\prime}$ is the unique connected component of $\operatorname{Conf}_{C_{n}}^{\text {fin }}\left(\widetilde{\mathbb{R}},\{ \pm \infty\} ; \mathcal{H}_{n}\right)$ that is not contractible. On the two remaining components the map $p_{*}$ is not a quasi-fibration as we shall see below (the fibres have distinct homotopy groups).

Lemma 8.7. Let $M$ be a graded $C_{n}$-module.

(1) If $M$ contains all (one or two) irreducible $C_{n}$-modules infinitely often then the $C_{n}$-action on $M$ extends to a graded $C_{n+1}$-action.

(2) Let $M_{0}$ be a graded irreducible $C_{n}$-module. Then there is a graded vector space $R$, the multiplicity space, such that $M$ is isomorphic to the graded tensor product $M_{0} \otimes R$. In the case $n \not \equiv 3(\bmod 4)$ we may, and shall, assume that $R$ is concentrated in even degree.

(3) With this notation, the grading preserving Clifford linear orthogonal group $O_{C_{n}}(M)$ is isomorphic to

$$
O_{C_{n}}(M) \cong O(R) \cong O\left(R^{\mathrm{ev}}\right) \times O\left(R^{\text {odd }}\right)
$$

In particular, this group is contractible (by Kuiper's theorem) if and only if the multiplicity spaces $R^{\mathrm{ev}}$ and $R^{\text {odd }}$ are both either zero or infinitedimensional. This is equivalent to $M$ containing either only one type of graded irreducible $C_{n}$-module, or containing both infinitely often.

Proof. There are two cases to consider for proving (1): If only one graded irreducible $C_{n}$-module exists, then take any irreducible $C_{n+1}$-module $M_{0}$ and restrict it to $C_{n}$. It is clear that over $C_{n}, M$ must be given by infinitely many copies of $M_{0}$.

Let's say there are two graded irreducible $C_{n}$-modules $M_{0}, M_{1}$ and hence $n$ is divisible by 4. By assumption, $M$ is the sum of infinitely many copies of $M_{0} \perp M_{1}$. It then suffices to show that $M_{0} \perp M_{1}$ has an $C_{n+1}$-action. We first claim that $M_{1} \cong M_{0}^{\mathrm{op}}$, i.e., $M_{1}$ is obtained from $M_{0}$ by flipping the grading. Using the characterization of ungraded $C_{n-1}$-modules given in Lemma 8.4, it suffices to show that the volume element $\tilde{e}_{1} \cdots \tilde{e}_{n-1}$ acts with different sign on $M_{0}^{\text {ev }}$ and $M_{0}^{\text {odd }}$. Here $\tilde{e}_{i}=e_{i} e_{n}$ are the usual generators of $C_{n-1} \cong C_{n}^{\text {ev }}$. Since $n$ is divisible by 4 , it follows that

$$
\tilde{e}_{1} \cdots \tilde{e}_{n-1}=\left(e_{1} e_{n}\right) \cdots\left(e_{n-1} e_{n}\right)=e_{1} \cdots e_{n}=: e
$$

is also the volume element in $C_{n}$. Writing $M_{0}^{\text {odd }}=e_{i} \cdot M_{0}^{\text {ev }}$ for some $i$, our claim follow from $e e_{i}=-e_{i} e$. Finally, the module $M_{0} \perp M_{0}^{\text {op }}$ has a $C_{n+1}$-action given by the element $e_{n+1}=f \alpha$, where $f$ flips the two summands and $\alpha$ is the grading involution. 
For part (2) one again needs to know that in the case that there are two graded irreducible $C_{n}$-modules $M_{0}, M_{1}$, they differ from each other by flipping the grading. This was proven above. Part (3) is obvious.

Proof of Theorem 8.5. We begin by proving that the fibers of $p$ are contractible. Fix $V \in \operatorname{Conf}_{n}^{\text {fin }}$. The fiber $p^{-1}(V)$ consists of all $W \in \operatorname{Conf}_{n}^{\prime}$ such that $V_{\lambda}=W_{\lambda}$ for $\lambda \in \mathbb{R}$ and $V_{\infty}$ is the orthogonal sum of $W_{\infty}$ and $W_{-\infty}$. Since $W_{-\infty}=\alpha\left(W_{\infty}\right)$, where $\alpha$ is the grading involution on $\mathcal{H}_{n}$, we may identify $p^{-1}(V)$ with the space of decompositions of the graded $C_{n}$-module $V_{\infty}$ of the form $V_{\infty}=W_{\infty} \perp \alpha\left(W_{\infty}\right)$, where $W_{\infty}$ is an ungraded $C_{n}$-submodule of $V_{\infty}$ that for $n \equiv 3(\bmod 4)$ satisfies the (AS) condition: both irreducible $C_{n}$-modules appear infinitely often in $W_{\infty}$.

Without the (AS) condition, it is straightforward to show that this space of decompositions of $V_{\infty}$ is homeomorphic to the following space of $C_{n+1}$-structures on $V_{\infty}$ :

$$
\widetilde{\mathfrak{C}}_{n+1}\left(V_{\infty}\right):=\left\{e_{n+1} \in O\left(V_{\infty}\right) \mid e_{n+1}^{2}=-\mathbb{1}, e_{n+1} e_{i}=-e_{i} e_{n+1}, i=1, \ldots, n\right\}
$$

Namely, given $e_{n+1}$, one can define $W_{ \pm \infty}$ to be the $( \pm 1)$-eigenspaces of $e_{n+1} \alpha$ (and vice versa). Under this correspondence, the (AS) condition translates into the requirement that $e_{n+1}$ defines a $C_{n+1}$ module structure on $V_{\infty}$ which contains both graded irreducibles infinitely often. We denote this subspace of $\widetilde{\mathfrak{C}}_{n+1}\left(V_{\infty}\right)$ simply by $\mathfrak{C}_{n+1}\left(V_{\infty}\right)$ and observe that all these module structures $e_{n+1}$ on $V_{\infty}$ are isomorphic.

We show in the following 4 steps that the fibre $p^{-1}(V) \approx \mathfrak{C}_{n+1}\left(V_{\infty}\right)$ is contractible under our assumptions.

Step 1. By our basic assumption, the ambient Hilbert space $\mathcal{H}_{n}$ contains all graded irreducible $C_{n}$-modules infinitely often. Since $V$ was a finite configuration to start with, it follows that $V_{\infty}$ has the same property and by part (1) of Lemma 8.7 it follows that $\mathfrak{C}_{n+1}\left(V_{\infty}\right)$ is not empty.

Step 2. Since any two points in $\mathfrak{C}_{n+1}\left(V_{\infty}\right)$ lead to $C_{n+1}$-module structures on $V_{\infty}$ that are isomorphic, the orthogonal group $O_{C_{n}}\left(V_{\infty}\right)$ acts transitively (by conjugation) on $\mathfrak{C}_{n+1}\left(V_{\infty}\right)$. The stabilizer of a particular $C_{n+1}$-structure is $O_{C_{n+1}}\left(V_{\infty}\right)$ and hence

$$
\mathfrak{C}_{n+1}\left(V_{\infty}\right) \approx O_{C_{n}}\left(V_{\infty}\right) / O_{C_{n+1}}\left(V_{\infty}\right)
$$

We shall show that this space is contractible, as a quotient of two contractible groups.

Step 3. As a $C_{n}$-module, $V_{\infty}$ contains both graded irreducible $C_{n}$-modules infinitely often, that's what we need by part (3) of Lemma 8.7 for the contractibility of the larger group $O_{C_{n}}\left(V_{\infty}\right)$.

Step 4. For the smaller group $O_{C_{n+1}}\left(V_{\infty}\right)$, the (AS) condition tells us again that the assumptions of part (3) of Lemma 8.7 are satisfied.

To finish the proof of Theorem 8.5, it remains to show that $p$ is indeed a quasifibration. We will use the criterion in Theorem 8.10 but first we give the relevant definitions.

Definition 8.8. A map $p: E \rightarrow B$ is a quasi-fibration if for all $b \in B, i \in \mathbb{N}$ and $e \in p^{-1}(b), p$ induces an isomorphism

$$
\pi_{i}\left(E, p^{-1}(b), e\right) \stackrel{\cong}{\rightrightarrows} \pi_{i}(B, b) .
$$


From the long exact sequence of homotopy groups for a pair it follows that $p$ is a quasi-fibration exactly if there is a long exact homotopy sequence connecting fibre, total space and base space of $p$, just like for a fibration. However, $p$ does not need to have any (path) lifting properties as the following example shows.

Example 8.9. The prototypical example of a quasi-fibration that is not a fibration is the projection of a 'step'

$$
(-\infty, 0] \times\{0\} \cup\{0\} \times[0,1] \cup[0, \infty) \times\{1\} \subset \mathbb{R}^{2}
$$

onto the $x$-axis. Even though all fibers have the same homotopy type (they are contractible), the map doesn't have the lifting property of a fibration, since it is impossible to lift a path that passes through the origin.

The following sufficient condition for a map to be a quasi-fibration is an easy consequence of the results of [7]:

Theorem 8.10. Let $p: Y \rightarrow X$ be a continuous map between Hausdorff spaces and $X_{0} \subset X_{1} \subset X_{2} \subset \cdots$ an increasing sequence of closed subsets of $X$ s.t. $X=\operatorname{colim}_{i \rightarrow \infty} X_{i}$. Assume further that for all $i \geq 0$ the map $\left.p\right|_{Y_{i+1} \backslash Y_{i}}$ is a Serre fibration, where $Y_{i}:=p^{-1}\left(X_{i}\right)$, and that there exists an open neighborhood $N_{i}$ of $X_{i}$ in $X_{i+1}$ and homotopies $d_{t}=d_{t}^{(i)}: N_{i} \rightarrow N_{i}$ and $D_{t}^{(i)}=D_{t}: p^{-1}\left(N_{i}\right) \rightarrow p^{-1}\left(N_{i}\right)$ s.t.

(1) $D$ covers $d$, i.e., $p \circ D_{t}=d_{t} \circ p$ for all $t$.

(2) $D_{0}=\mathrm{id}, D_{t}\left(Y_{i}\right) \subset Y_{i}$ for all $t$, and $D_{1}\left(p^{-1}\left(N_{i}\right)\right) \subset Y_{i}$

(3) For every $x \in N_{i}$, the map $D_{1}: p^{-1}(x) \rightarrow p^{-1}\left(d_{1}(x)\right)$ is a weak homotopy equivalence.

Then $p$ is a quasi-fibration.

Proof. According to Satz 2.15 in [7] $p$ is a quasi-fibration provided that $\left.p\right|_{Y_{i}}$ is a quasi-fibration for all $i \geq 0$. To see this, we proceed by induction on $i$. It is clear that $\left.p\right|_{Y_{0}}$ is a quasi-fibration, since, by assumption, it is a Serre fibration. Now assume that we already know that $\left.p\right|_{Y_{i}}$ is a quasi-fibration. Applying Hilfssatz 2.10 in [7] with $B=N_{i}, B^{\prime}=X_{i}, q=\left.p\right|_{p^{-1}\left(N_{i}\right)}, D=D$, and $d=d$, implies that $\left.p\right|_{p^{-1}\left(N_{i}\right)}$ is a quasi-fibration. Now, applying the Korollar of Satz 2.2 in [7] with $U=N_{i+1}$ and $V=Y_{i+1} \backslash Y_{i}$ we see that $\left.p\right|_{Y_{i+1}}$ is a quasi-fibration. (Note that $p$ is a quasi-fibration over $U \cap V$, since it is even a Serre fibration.)

Now, in order to apply Theorem 8.10 we filter Conf $f_{n}^{\text {fin }}$ by the closed subspaces

$$
X_{i}:=\left\{V \in \operatorname{Conf}_{n}^{\text {fin }} \mid \operatorname{dim}(V):=\operatorname{dim}_{C_{n}}\left(\bigoplus_{\lambda \in \mathbb{R}} V_{\lambda}\right) \leq 2 i\right\} .
$$

For each $i$ consider the continuous function

$$
L_{i}: X_{i+1} \rightarrow[0, \infty], \quad V \mapsto L_{i}(V):=\sup \left\{r \mid \operatorname{dim}_{C_{n}}\left(\bigoplus_{\lambda \in(-r, r)} V_{\lambda}\right) \leq 2 i\right\} .
$$

Clearly, $L_{i}^{-1}(\infty)=X_{i}$. Define $N_{i}:=L_{i}^{-1}((1, \infty])$. The homotopies $d^{(i)}$ and $D^{(i)}$ are now easy to find. Namely, consider the homotopies on $\operatorname{Conf}_{n}^{\text {fin }}$ and Conf $_{n}^{\prime}$ induced by the family of maps $h_{t}$ defined in the proof of Proposition 4.6 (in the case of Conf $_{n}^{\prime}$, note that the formula defining $h_{t}$ indeed also determines a homotopy on $\widetilde{\mathbb{R}}$ and hence on Conf $_{n}^{\prime}$ ). It is easy to check that almost all assumptions of the DoldThom theorem are hold: (1) and (2) obviously satisfied. Condition (3) is trivial, 
since all fibers are contractible. The map $\left.p\right|_{X_{i+1} \backslash X_{i}}$ is a fiber bundle, in particular, it is a Serre fibration. The crucial point in the proof of this is that dimension $\operatorname{dim}(V)$ of configurations in $X_{i+1} \backslash X_{i}$ is constant. This makes it possible to choose an open neighborhood $N$ of $V$ in $X_{i+1} \backslash X_{i}$ such that the orthogonal projection $P_{V_{\infty}}: W_{\infty} \rightarrow V_{\infty}$ is an isomorphism for all $W \in N$. It is easy to write down a local trivialization of $p$ over such an $N$; this shows that $\left.p\right|_{X_{i+1} \backslash X_{i}}$ is a fiber bundle.

The only condition in the Dold - Thom theorem that is violated is that $\operatorname{Conf}_{n}^{\mathrm{fin}}$ is not the colimit over the subspaces $X_{i}$ (cf. Remark 4.7). However, this is not a serious issue: we can endow $\operatorname{Conf}_{n}^{\text {fin }}$ and $\operatorname{Conf}_{n}^{\prime}$ with the colimit topologies with respect to the filtrations $X_{i}$ and $p^{-1}\left(X_{i}\right)$ and apply the Dold-Thom theorem to see that $p$ is a quasi-fibration in this case. It follows directly from the definition of a quasi-fibration that the same also holds if we consider the original topologies, since the identity maps $\operatorname{colim}_{i \rightarrow \infty} X_{i} \rightarrow \operatorname{Conf}_{n}^{\text {fin }}$ and $\operatorname{colim}_{i \rightarrow \infty} Y_{i} \rightarrow$ Conf $_{n}^{\prime}$ are homotopy equivalences (see Remark 4.7 for the $\operatorname{Conf}_{n}^{\text {fin }}$ case, the same argument works in the case $\operatorname{Conf}_{n}^{\prime}$ ). This completes the proof of Theorem 8.5.

\section{References}

1. M. F. Atiyah, R. Bott, and A. Shapiro, Clifford modules, Topology 3 (1964), suppl 1, 3-38.

2. M. F. Atiyah and I. M. Singer, Index theory for skew-adjoint Fredholm operators, Inst. Hautes Études Sci. Publ. Math. 37 (1969), 5-26.

3. M. Batchelor, The structure of super manifolds, Trans. Amer. Math. Soc. 253 (1979), 329338.

4. R. Bott, The stable homotopy of the classical groups, Ann. of Math. (2) 70 (1959), 313-337.

5. P. Cheung, Supersymmetric field theories and cohomology, Ph.D. dissertation, Stanford University, 2006, arXiv:0811.2267.

6. P. Deligne and J. W. Morgan, Notes on supersymmetry (following Joseph Bernstein), Quantum Fields and Strings: A Course for Mathematicians, Vol. 1, 2 (Princeton, NJ, 1996/1997), Amer. Math. Soc., Providence, RI, 1999, pp. 41-97.

7. A. Dold and R. Thom, Quasifaserungen und unendliche symmetrische Produkte, Ann. of Math. (2) 67 (1958), 239-281.

8. D. S. Freed, Five lectures on supersymmetry, Amer. Math. Soc., Providence, RI, 1999.

9. D. Grayson, Higher algebraic K-theory. II (after Daniel Quillen), Algebraic $K$-theory (Evanston, IL, 1976), Lecture Notes in Math., vol. 551, Springer, Berlin, 1976, pp. 217-240.

10. N. Higson and E. Guentner, Group $C^{*}$-algebras and $K$-theory, Noncommutative Geometry, Lecture Notes in Math., vol. 1831, Springer, Berlin, 2004, pp. 137-251.

11. H. Hohnhold, M. Kreck, S. Stolz, and P. Teichner, Differential forms and 0-dimensional supersymmetric field theories, preprint.

12. H. Hohnhold, S. Stolz, and P. Teichner, $K$-theory and 1-dimensional supersymmetric field theories, in progress.

13. M. Joachim, $K$-homology of $C^{*}$-categories and symmetric spectra representing $K$-homology, Math. Ann. 327 (2003), no. 4, 641-670.

14. M. Karoubi, K-theory: An introduction, Grundlehren Math. Wiss., vol. 226, Springer, Berlin, 1978.

15. G. Köthe, Topological vector spaces. I, Grundlehren Math. Wiss., vol. 159, Springer, New York, 1969.

16. _ Topological vector spaces. II, Grundlehren Math. Wiss., vol. 237, Springer, New York, 1979.

17. H. B. Lawson Jr. and M.-L. Michelsohn, Spin geometry, Princeton Math. Ser., vol. 38, Princeton Univ. Press, Princeton, NJ, 1989.

18. A. Libman, Orbit spaces, the normaliser decomposition and Minami's formula for compact Lie groups, preprint.

19. E. Markert, Connective 1-dimensional Euclidean field theories, Ph.D. thesis, University of Notre Dame, 2005. 
20. R. Meise and D. Vogt, Introduction to functional analysis, Oxford Grad. Texts Math., vol. 2, Oxford Univ. Press, New York, 1997.

21. J. Milnor, On spaces having the homotopy type of CW-complex, Trans. Amer. Math. Soc. 90 (1959), 272-280.

22. 1963.

23. R. S. Palais, On the homotopy type of certain groups of operators, Topology 3 (1965), 271279 .

24. M. Reed and B. Simon, Methods of modern mathematical physics. I: Functional analysis, 2nd ed., Academic Press Inc., New York, 1980.

25. W. Rudin, Functional analysis, 2nd ed., Internat. Ser. Pure Appl. Math., McGraw-Hill Inc., New York, 1991.

26. G. Segal, Categories and cohomology theories, Topology 13 (1974), 293-312.

27. $\quad K$-homology theory and algebraic $K$-theory, $K$-Theory and Operator Algebras (Athens, GA, 1975), Lecture Notes in Math., vol. 575, Springer, Berlin, 1977, pp. 113-127.

28. S. Stolz and P. Teichner, What is an elliptic object?, Topology, Geometry and Quantum Field Theory, London Math. Soc. Lecture Note Ser., vol. 308, Cambridge Univ. Press, Cambridge, 2004, pp. $247-343$.

29. _ Supersymmetric Euclidean field theories and generalized cohomology, preprint.

30. W. P. Thurston, Three-dimensional geometry and topology. Vol. 1, Princeton Math. Ser., vol. 35, Princeton Univ. Press, Princeton, NJ, 1997.

31. R. Wood, Banach algebras and Bott periodicity, Topology 4 (1965/1966), 371-389.

Statistisches Bundesamt, 65180 Wiesbaden, Germany

E-mail address: henninghohnhold@gmail.com

Department of Mathematics, University of Notre Dame, 255 Hurley Hall, Notre DAME, IN 46556-4618, USA

E-mail address: stolz.1@nd.edu

Department of Mathematics, University of California, Berkeley, CA, 94720-3840, USA

Current address: Max-Planck Institut für Mathematik, P.O.Box 7280, 53072 Bonn, Germany

E-mail address: teichner@mac.com 